\title{
23. NATURAL GAMMA RADIATION OF SEDIMENTS FROM THE WESTERN EQUATORIAL PACIFIC: LEG 7, GLOMAR CHALLENGER
}

\author{
E. L. Gealy, Scripps Institution of Oceanography, La Jolla, California
}

\section{INTRODUCTION}

The distribution of radionuclides with depth in marine sediments is poorly understood, and the study of the gamma radiation cores of sediments recovered by deep ocean drilling could provide a unique opportunity for studying some aspects of this distribution.

The D/V Glomar Challenger is equipped with a system wherein the total gamma rays emitted over a given period by cores are counted and recorded. Unfortunately, the system is a relatively "noisy" one, it has not been rigorously calibrated; and, it does not permit determination of the energy spectra of the gamma radiation.

However, on Leg 7 of the D/V Glomar Challenger the system provided data which permit some statements to be made concerning the general level of gamma radiation of the sediments penetrated.

This report summarizes and evaluates natural gamma radiation data collected on $\operatorname{Leg} 7$, and attempts to interpret some of the findings.

\section{METHODS}

The natural gamma radiation of almost all sections recovered on Leg 7 of the D/V Glomar Challenger was measured by passing the unsplit core section through a natural gamma scanning system. The system used consists of (1) four shielded 3 by 3 inch scintillation detectors located 90 degrees apart in a plane normal to the length of the core section; (2) an incremented time step core transport mechanism set to advance a section through three inches per step each 1.25 minutes; (3) two dual input, single channel analyzers to accumulate total count rate; (4) a timing unit to regulate both core transport and counting times; (5) a digital to analog converter to supply the analog count rate to two strip recorders; and (6) a printer which yields a tabulation of total count. Details of the system are described by Evans and Lucia (1970) and by Maxwell et al. (1970).

The unit includes neither a pulse-height analyzer nor a multi-channel analyzer, but measures the total count through a fixed window. Gamma rays having an energy equal to or greater than $0.3 \mathrm{Mev}$ are passed and counted.
The gamma ray scanning device aboard the Glomar Challenger has not been rigorously calibrated. However, in most cases, two controls were run before the natural gamma radiation was measured on each core. The first was an "air" standard wherein the counter was left running with nothing in the scanner for a series of 1.25 minute readings. The second was a "water" standard wherein a series of natural gamma radiation measurements were made of a section of plastic core liner filled with sea water.

An arithmetic mean of the measurements of the air and water controls was obtained for each series of tests. These means are listed next to the appropriate sections in Table 1 and are plotted for the appropriate sections as a function of depth on Figures 1 through 6 . Their frequency distribution is shown on Figure 10. The means of the water control measurements range from 1379 to 1556 counts per 1.25 minutes; 88 per cent of the means are between 1450 and 1525 counts; and the median of these values is 1495 . The mean air control measurements range from 1361 to 1616 counts per 1.25 minutes; 90 per cent of the controls are between 1400 and 1475 , and the median of these values is 1445. The higher measurements for the water controls probably reflect radioactivity of the plastic liner and the seawater it contains.

When variations in the measurements of the controls are compared with the measurement of the gamma radiation of sections made immediately after the standards were run, no consistent correlation between background count and counts on the cores can be seen, even when the sediments show very low levels of radiation. Why there is no correlation is uncertain, but it may be that the cores provide more shielding against extraneous radiation in the laboratory entering into the access hole in the lead shielding of the system than does the open hole or even the water blank. The air controls reflect even less shielding effect than the water, and the wider range of mean values for the air controls reflects this. If these conditions prevail, the actual background count for sediment cores will be lower and less erratic than either the water or air controls.

On Leg 4 at Site 29 (Gealy and Gerard, 1970), in-situ gamma radiation was measured with a calibrated logging tool. When the values so obtained were 
TABLE 1

Natural Gamma Radiation

\begin{tabular}{|c|c|c|c|c|c|c|c|c|c|c|c|c|c|c|}
\hline Leg & Hole & Core & Section & $\begin{array}{l}\text { Depth Below } \\
\text { Sea Floor } \\
\text { (Meters) }\end{array}$ & $\begin{array}{c}\gamma^{\mathrm{a}} \\
\text { Gamma } \\
\text { Radiation }\end{array}$ & $\begin{array}{c}\text { Mean } \\
\text { Water } \\
\text { Control }\end{array}$ & \begin{tabular}{|c|} 
Mean \\
Air \\
Control
\end{tabular} & $\begin{array}{c}\phi \\
\text { Porosity } \\
\text { Fraction }\end{array}$ & $\begin{array}{c}1-\phi \\
\text { Solid } \\
\text { Fraction }\end{array}$ & $\gamma /(1-\phi)$ & $\begin{array}{c}\mathrm{CO}_{3} \\
\text { Weight } \\
\text { Fraction }\end{array}$ & $\begin{array}{l}1-\mathrm{CO}_{3} \\
\text { Weight } \\
\text { Fraction }\end{array}$ & $\begin{array}{c}(1-\phi)\left(1-\mathrm{CO}_{3}\right) \\
\text { Weight } \\
\text { Fraction }\end{array}$ & $\gamma /(1-\phi)\left(1-\mathrm{CO}_{3}\right)$ \\
\hline \multicolumn{15}{|c|}{ Site 61} \\
\hline 7 & 61.1 & 1 & 1 & 83.75 & 344 & 1379 & 1361 & 0.495 & 0.505 & 681 & 0 & 1.000 & 0.505 & 681 \\
\hline 7 & 61.1 & 1 & 2 & 85.25 & 519 & 1379 & 1361 & 0.408 & 0.592 & 876 & 0 & 1.000 & 0.592 & 876 \\
\hline \multicolumn{15}{|c|}{ Site 62} \\
\hline 7 & 62 & 1 & 1 & 91.75 & 405 & & 1429 & 0.604 & 0.396 & 1023 & 0.848 & 0.152 & 0.060 & 6729 \\
\hline 7 & 62 & 1 & 1 & 93.25 & 341 & & 1429 & 0.618 & 0.382 & 894 & 0.823 & 0.177 & 0.068 & 5050 \\
\hline 7 & 62 & 1 & 3 & 94.75 & 341 & & 1429 & 0.608 & 0.392 & 872 & 0.875 & 0.125 & 0.049 & 6973 \\
\hline 7 & 62 & 1 & 4 & 96.25 & 301 & & 1429 & 0.623 & 0.377 & 798 & 0.848 & 0.152 & 0.057 & 5250 \\
\hline 7 & 62 & 1 & 5 & 97.75 & 350 & & 1429 & 0.624 & 0.376 & 932 & 0.793 & 0.207 & 0.078 & 4504 \\
\hline 7 & 62 & 1 & 6 & 99.25 & 390 & & 1429 & 0.629 & 0.371 & 1052 & 0.856 & 0.144 & 0.053 & 7307 \\
\hline 7 & 62 & 2 & 1 & 205.75 & 177 & 1509 & 1451 & 0.768 & 0.232 & 765 & 0.895 & 0.105 & 0.024 & 7231 \\
\hline 7 & 62 & 2 & 2 & 207.25 & 228 & 1509 & 1451 & 0.584 & 0.416 & 549 & 0.896 & 0.104 & 0.043 & 5234 \\
\hline 7 & 62 & 2 & 3 & 208.75 & 196 & 1509 & 1451 & 0.584 & 0.416 & 471 & 0.870 & 0.130 & 0.054 & 3621 \\
\hline 7 & 62 & 2 & 4 & 210.25 & 205 & 1509 & 1451 & 0.585 & 0.415 & 494 & 0.895 & 0.105 & 0.044 & 4704 \\
\hline 7 & 62 & 2 & 5 & 211.75 & 214 & 1509 & 1451 & 0.581 & 0.419 & 511 & 0.909 & 0.091 & 0.038 & 5610 \\
\hline 7 & 62 & 2 & 6 & 213.25 & 222 & 1509 & 1451 & 0.582 & 0.418 & 533 & 0.938 & 0.062 & 0.026 & 8590 \\
\hline 7 & 62 & 3 & 1 & 299.75 & 206 & 1462 & 1449 & 0.572 & 0.428 & 481 & 0.910 & 0.090 & 0.039 & 5350 \\
\hline 7 & 62 & 3 & 2 & 301.25 & 182 & 1462 & 1449 & 0.586 & 0.414 & 440 & 0.970 & 0.030 & 0.012 & 14654 \\
\hline 7 & 62 & 3 & 2 & 302.25 & 186 & 1462 & 1449 & 0.519 & 0.421 & 442 & 0.886 & 0.114 & 0.048 & 3878 \\
\hline 7 & 62 & 3 & 4 & 304.25 & 198 & 1462 & 1449 & 0.576 & 0.424 & 466 & 0.908 & 0.092 & 0.039 & 5054 \\
\hline 7 & 62 & 3 & 5 & 305.75 & 205 & 1462 & 1449 & 0.558 & 0.442 & 464 & 0.940 & 0.060 & 0.027 & 7740 \\
\hline 7 & 62 & 3 & 6 & 307.25 & 215 & 1462 & 1449 & 0.581 & 0.419 & 513 & 0.883 & 0.117 & 0.049 & 4335 \\
\hline 7 & 62 & 4 & 1 & 395.75 & 273 & 1487 & 1429 & 0.567 & 0.433 & 631 & 0.783 & 0.217 & 0.094 & 2910 \\
\hline 7 & 62 & 4 & 2 & 397.25 & 296 & 1490 & 1393 & 0.496 & 0.304 & 587 & 0.857 & 0.143 & 0.072 & 4107 \\
\hline 7 & 62 & 4 & 3 & 398.75 & 262 & 1490 & 1393 & 0.501 & 0.499 & 525 & 0.828 & 0.172 & 0.086 & 3052 \\
\hline 7 & 62 & 4 & 4 & 400.25 & 249 & 1490 & 1393 & 0.599 & 0.401 & 620 & 0.810 & 0.190 & 0.076 & 3262 \\
\hline
\end{tabular}


TABLE 1 - Continued

\begin{tabular}{|c|c|c|c|c|c|c|c|c|c|c|c|c|c|c|}
\hline Leg & Hole & Core & Section & $\begin{array}{l}\text { Depth Below } \\
\text { Sea Floor } \\
\text { (Meters) }\end{array}$ & $\begin{array}{c}\gamma^{\mathrm{a}} \\
\text { Gamma } \\
\text { Radiation }\end{array}$ & $\begin{array}{c}\text { Mean } \\
\text { Water } \\
\text { Control } \\
\end{array}$ & $\begin{array}{c}\text { Mean } \\
\text { Air } \\
\text { Control }\end{array}$ & $\begin{array}{c}\phi \\
\text { Porosity } \\
\text { Fraction } \\
\end{array}$ & $\begin{array}{c}1-\phi \\
\text { Solid } \\
\text { Fraction }\end{array}$ & $\gamma /(1-\phi)$ & $\begin{array}{c}\mathrm{CO}_{3} \\
\text { Weight } \\
\text { Fraction }\end{array}$ & $\begin{array}{c}1-\mathrm{CO}_{3} \\
\text { Weight } \\
\text { Fraction }\end{array}$ & $\begin{array}{c}(1-\phi)\left(1-\mathrm{CO}_{3}\right) \\
\text { Weight } \\
\text { Fraction }\end{array}$ & $\gamma /(1-\phi)\left(1-\mathrm{CO}_{3}\right)$ \\
\hline \multicolumn{15}{|c|}{ Site 62 - Continued } \\
\hline 7 & 62 & 4 & 5 & 401.75 & 206 & 1490 & 1393 & 0.522 & 0.478 & 556 & 0.835 & 0.165 & 0.079 & 3373 \\
\hline 7 & 62 & 4 & 6 & 403.25 & 255 & 1490 & 1393 & 0.576 & 0.424 & 602 & 0.797 & 0.203 & 0.086 & 2957 \\
\hline 7 & 62 & 5 & 2 & 492.25 & 112 & 1458 & 1387 & 0.503 & 0.497 & 226 & 0.891 & 0.109 & 0.054 & 2073 \\
\hline 7 & 62 & 5 & 3 & 493.75 & 81 & 1458 & 1387 & 0.482 & 0.518 & 157 & 0.886 & 0.114 & 0.059 & 1373 \\
\hline 7 & 62 & 5 & 4 & 495.25 & 120 & 1458 & 1387 & 0.449 & 0.551 & 217 & 0.927 & 0.073 & 0.040 & 2975 \\
\hline 7 & 62.1 & 1 & 2 & 8.25 & 918 & 1505 & 1438 & 0.768 & 0.232 & 3959 & 0.621 & 0.379 & 0.088 & 10446 \\
\hline 7 & 62.1 & 1 & 3 & 9.75 & 557 & 1505 & 1438 & 0.711 & 0.289 & 1927 & 0.565 & 0.435 & 0.126 & 4429 \\
\hline 7 & 62.1 & 4 & 1 & 34.75 & 385 & 1486 & 1429 & 0.719 & 0.281 & 1368 & 0.722 & 0.278 & 0.078 & 4921 \\
\hline 7 & 62.1 & 4 & 2 & 36.25 & 367 & 1486 & 1429 & 0.708 & 0.294 & 1250 & 0.710 & 0.290 & 0.085 & 4310 \\
\hline 7 & 62.1 & 4 & 4 & 39.25 & 369 & 1486 & 1429 & 0.688 & 0.312 & 1184 & 0.798 & 0.202 & 0.063 & 5852 \\
\hline 7 & 62.1 & 4 & 5 & 40.75 & 321 & 1486 & 1429 & 0.673 & 0.327 & 981 & 0.801 & 0.199 & 0.065 & 4931 \\
\hline 7 & 62.1 & 4 & 6 & 42.25 & 343 & 1486 & 1429 & 0.676 & 0.324 & 1058 & 0.758 & 0.242 & 0.078 & 4372 \\
\hline 7 & 62.1 & 6 & 1 & 54.75 & 360 & 1470 & 1411 & 0.715 & 0.285 & 1265 & 0.501 & 0.499 & 0.142 & 2535 \\
\hline 7 & 62.1 & 6 & 2 & 56.25 & 347 & 1470 & 1411 & 0.712 & 0.288 & 1206 & 0.810 & 0.190 & 0.055 & 6346 \\
\hline 7 & 62.1 & 6 & 3 & 57.75 & 346 & 1470 & 1411 & 0.709 & 0.291 & 1188 & 0.736 & 0.264 & 0.077 & 4501 \\
\hline 7 & 62.1 & 6 & 4 & 59.25 & 345 & 1470 & 1411 & 0.700 & 0.300 & 1149 & 0.591 & 0.409 & 0.123 & 2809 \\
\hline 7 & 62.1 & 6 & 5 & 60.75 & 359 & 1470 & 1411 & 0.696 & 0.304 & 1183 & 0.743 & 0.257 & 0.078 & 4604 \\
\hline 7 & 62.1 & 6 & 6 & 62.25 & 314 & 1470 & 1411 & 0.665 & 0.335 & 938 & 0.760 & 0.240 & 0.080 & 3907 \\
\hline 7 & 62.1 & 7 & 1 & 63.75 & 347 & 1514 & 1451 & 0.688 & 0.312 & 1113 & 0.675 & 0.325 & 0.101 & 3426 \\
\hline 7 & 62.1 & 7 & 2 & 65.25 & 346 & 1502 & 1485 & 0.657 & 0.343 & 1007 & 0.630 & 0.350 & 0.120 & 2877 \\
\hline 7 & 62.1 & 7 & 3 & 66.75 & 404 & 1502 & 1485 & 0.679 & 0.321 & 1258 & 0.743 & 0.257 & 0.082 & 4896 \\
\hline 7 & 62.1 & 7 & 4 & 68.25 & 396 & 1502 & 1485 & 0.691 & 0.309 & 1280 & 0.618 & 0.382 & 0.118 & 3351 \\
\hline 7 & 62.1 & 7 & 5 & 69.75 & 397 & 1502 & 1485 & 0.644 & 0.356 & 1115 & 0.883 & 0.117 & 0.042 & 9529 \\
\hline 7 & 62.1 & 7 & 6 & 71.25 & 388 & 1502 & 1485 & 0.675 & 0.325 & 1195 & 0.770 & 0.230 & 0.075 & 5197 \\
\hline 7 & 62.1 & 8 & 2 & 74.25 & 349 & 1507 & 1455 & 0.645 & 0.355 & 984 & 0.648 & 0.352 & 0.125 & 2794 \\
\hline
\end{tabular}


TABLE 1 - Continued

\begin{tabular}{|c|c|c|c|c|c|c|c|c|c|c|c|c|c|c|}
\hline Leg & Hole & Core & Section & $\begin{array}{l}\text { Depth Below } \\
\text { Sea Floor } \\
\text { (Meters) }\end{array}$ & $\begin{array}{c}\gamma^{\mathrm{a}} \\
\text { Gamma } \\
\text { Radiation }\end{array}$ & $\begin{array}{c}\text { Mean } \\
\text { Water } \\
\text { Control }\end{array}$ & $\begin{array}{c}\text { Mean } \\
\text { Air } \\
\text { Control }\end{array}$ & $\begin{array}{c}\phi \\
\text { Porosity } \\
\text { Fraction }\end{array}$ & $\begin{array}{c}1-\phi \\
\text { Solid } \\
\text { Fraction }\end{array}$ & $\gamma /(1-\phi)$ & $\begin{array}{c}\mathrm{CO}_{3} \\
\text { Weight } \\
\text { Fraction }\end{array}$ & $\begin{array}{l}1-\mathrm{CO}_{3} \\
\text { Weight } \\
\text { Fraction }\end{array}$ & $\begin{array}{c}(1-\phi)\left(1-\mathrm{CO}_{3}\right) \\
\text { Weight } \\
\text { Fraction }\end{array}$ & $\gamma /(1-\phi)\left(1-\mathrm{CO}_{3}\right)$ \\
\hline \multicolumn{15}{|c|}{ Site 62 - Continued } \\
\hline 7 & 62.1 & 8 & 3 & 75.75 & 371 & 1507 & 1455 & 0.655 & 0.345 & 1075 & 0.681 & 0.319 & 0.110 & 3371 \\
\hline 7 & 62.1 & 8 & 4 & 77.25 & 397 & 1507 & 1455 & 0.642 & 0.358 & 1108 & 0.812 & 0.188 & 0.067 & 5897 \\
\hline 7 & 62.1 & 8 & 5 & 78.75 & 448 & 1507 & 1455 & 0.651 & 0.349 & 1286 & 0.736 & 0.264 & 0.092 & 4870 \\
\hline 7 & 62.1 & 8 & 6 & 80.25 & 385 & 1507 & 1455 & 0.644 & 0.356 & 1081 & 0.729 & 0.271 & 0.097 & 3988 \\
\hline 7 & 62.1 & 9 & 2 & 83.25 & 408 & 1522 & 1454 & 0.659 & 0.341 & 1194 & 0.791 & 0.209 & 0.071 & 5715 \\
\hline 7 & 62.1 & 9 & 4 & 86.25 & 409 & 1500 & 1443 & 0.663 & 0.337 & 1214 & 0.625 & 0.375 & 0.126 & 3236 \\
\hline 7 & 62.1 & 9 & 3 & 84.75 & 398 & 1500 & 1443 & 0.636 & 0.364 & 1093 & 0.740 & 0.260 & 0.095 & 4205 \\
\hline 7 & 62.1 & 10 & 1 & 92.75 & 355 & 1518 & 1449 & 0.673 & 0.327 & 1086 & 0.478 & 0.522 & 0.171 & 2080 \\
\hline 7 & 62.1 & 10 & 2 & 94.25 & 362 & 1485 & 1438 & 0.688 & 0.312 & 1161 & 0.760 & 0.240 & 0.075 & 4838 \\
\hline 7 & 62.1 & 10 & 3 & 95.75 & 508 & 1485 & 1438 & 0.673 & 0.327 & 1553 & 0.655 & 0.345 & 0.113 & 4500 \\
\hline 7 & 62.1 & 10 & 4 & 97.25 & 427 & 1485 & 1438 & 0.674 & 0.326 & 1311 & 0.759 & 0.231 & 0.075 & 5675 \\
\hline 7 & 62.1 & 10 & 5 & 98.75 & 489 & 1485 & 1438 & 0.665 & 0.335 & 1459 & 0.725 & 0.275 & 0.092 & 5304 \\
\hline 7 & 62.1 & 10 & 6 & 100.25 & 413 & 1485 & 1438 & 0.638 & 0.362 & 1142 & 0.770 & 0.230 & 0.083 & 4955 \\
\hline 7 & 62.1 & 11 & 1 & 101.75 & 387 & 1517 & 1438 & 0.629 & 0.371 & 1043 & 0.785 & 0.215 & 0.080 & 4852 \\
\hline 7 & 62.1 & 11 & 2 & 103.25 & 364 & 1517 & 1417 & 0.653 & 0.347 & 1049 & 0.785 & 0.215 & 0.075 & 4877 \\
\hline 7 & 62.1 & 11 & 3 & 104.75 & 388 & 1517 & 1417 & 0.640 & 0.360 & 1078 & 0.792 & 0.208 & 0.075 & 5183 \\
\hline 7 & 62.1 & 11 & 4 & 106.25 & 393 & 1517 & 1417 & 0.639 & 0.361 & 1087 & 0.689 & 0.311 & 0.112 & 3495 \\
\hline 7 & 62.1 & 11 & 5 & 107.75 & 455 & 1517 & 1417 & 0.644 & 0.356 & 1279 & 0.769 & 0.231 & 0.082 & 5539 \\
\hline 7 & 62.1 & 11 & 6 & 109.25 & 357 & 1517 & 1417 & 0.648 & 0.352 & 1014 & 0.776 & 0.224 & 0.079 & 4526 \\
\hline 7 & 62.1 & 12 & 1 & 110.75 & 361 & 1477 & 1486 & 0.645 & 0.355 & 1017 & 0.789 & 0.211 & 0.075 & 4819 \\
\hline 7 & 62.1 & 12 & 2 & 112.25 & 324 & 1502 & 1432 & 0.638 & 0.362 & 895 & 0.836 & 0.164 & 0.059 & 5456 \\
\hline 7 & 62.1 & 12 & 3 & 113.75 & 319 & 1502 & 1432 & 0.616 & 0.384 & 832 & 0.840 & 0.160 & 0.061 & 5197 \\
\hline 7 & 62.1 & 12 & 4 & 115.25 & 339 & 1502 & 1432 & 0.604 & 0.396 & 855 & 0.835 & 0.165 & 0.065 & 5182 \\
\hline 7 & 62.1 & 12 & 5 & 116.75 & 318 & 1502 & 1432 & 0.629 & 0.371 & 856 & 0.824 & 0.176 & 0.065 & 4856 \\
\hline 7 & 62.1 & 12 & 6 & 118.25 & 348 & 1502 & 1432 & 0.609 & 0.391 & 890 & 0.862 & 0.138 & 0.054 & 6449 \\
\hline
\end{tabular}


TABLE 1 - Continued

\begin{tabular}{|c|c|c|c|c|c|c|c|c|c|c|c|c|c|c|}
\hline Leg & Hole & Core & Section & $\begin{array}{l}\text { Depth Below } \\
\text { Sea Floor } \\
\text { (Meters) }\end{array}$ & $\begin{array}{c}\gamma^{\mathrm{a}} \\
\text { Gamma } \\
\text { Radiation }\end{array}$ & $\begin{array}{l}\text { Mean } \\
\text { Water } \\
\text { Control }\end{array}$ & $\begin{array}{l}\text { Mean } \\
\text { Air } \\
\text { Control }\end{array}$ & $\begin{array}{c}\phi \\
\text { Porosity } \\
\text { Fraction }\end{array}$ & $\begin{array}{c}1-\phi \\
\text { Solid } \\
\text { Fraction }\end{array}$ & $\gamma /(1-\phi)$ & $\begin{array}{c}\mathrm{CO}_{3} \\
\text { Weight } \\
\text { Fraction }\end{array}$ & $\begin{array}{l}1-\mathrm{CO}_{3} \\
\text { Weight } \\
\text { Fraction }\end{array}$ & $\begin{array}{c}(1-\phi)\left(1-\mathrm{CO}_{3}\right) \\
\text { Weight } \\
\text { Fraction }\end{array}$ & $\gamma /(1-\phi)\left(1-\mathrm{CO}_{3}\right)$ \\
\hline \multicolumn{15}{|c|}{ Site 62-Continued } \\
\hline 7 & 62.1 & 13 & 5 & 125.75 & 312 & 1493 & 1455 & 0.646 & 0.354 & 881 & 0.840 & 0.160 & 0.057 & 5504 \\
\hline 7 & 62.1 & 14 & 1 & 129.75 & 274 & 1516 & 1449 & 0.651 & 0.349 & 786 & 0.859 & 0.141 & 0.049 & 5577 \\
\hline 7 & 62.1 & 14 & 2 & 131.25 & 273 & 1490 & 1486 & 0.686 & 0.314 & 869 & 0.845 & 0.155 & 0.049 & 5604 \\
\hline 7 & 62.1 & 14 & 3 & 132.75 & 274 & 1490 & 1486 & 0.643 & 0.357 & 766 & 0.866 & 0.134 & 0.048 & 5716 \\
\hline 7 & 62.1 & 14 & 4 & 134.25 & 270 & 1490 & 1486 & 0.637 & 0.363 & 744 & 0.778 & 0.222 & 0.081 & 3351 \\
\hline 7 & 62.1 & 14 & 5 & 135.75 & 265 & 1490 & 1486 & 0.684 & 0.336 & 789 & 0.856 & 0.144 & 0.048 & 5432 \\
\hline 7 & 62.1 & 14 & 6 & 137.25 & 305 & 1490 & 1486 & 0.722 & 0.278 & 1095 & 0.843 & 0.157 & 0.044 & 6977 \\
\hline 7 & 62.1 & 15 & 1 & 138.75 & 296 & 1505 & 1446 & 0.613 & 0.387 & 765 & 0.418 & 0.582 & 0.225 & 1315 \\
\hline 7 & 62.1 & 15 & 2 & 140.25 & 288 & 1478 & 1439 & 0.623 & 0.377 & 765 & 0.856 & 0.144 & 0.054 & 5310 \\
\hline 7 & 62.1 & 15 & 3 & 141.75 & 297 & 1478 & 1439 & 0.622 & 0.378 & 787 & 0.856 & 0.134 & 0.051 & 5875 \\
\hline 7 & 62.1 & 15 & 4 & 143.25 & 336 & 1478 & 1439 & 0.621 & 0.379 & 885 & 0.601 & 0.399 & 0.151 & 2219 \\
\hline 7 & 62.1 & 15 & 5 & 144.75 & 310 & 1478 & 1439 & 0.619 & 0.381 & 815 & 0.676 & 0.324 & 0.123 & 2517 \\
\hline 7 & 62.1 & 15 & 6 & 146.25 & 318 & 1478 & 1439 & 0.613 & 0.387 & 820 & 0.829 & 0.171 & 0.066 & 4797 \\
\hline 7 & 62.1 & 16 & 1 & 147.75 & 333 & 1520 & 1453 & 0.635 & 0.365 & 911 & 0.901 & 0.099 & 0.036 & 9204 \\
\hline 7 & 62.1 & 16 & 2 & 149.25 & 302 & 1520 & 1453 & 0.627 & 0.373 & 809 & 0.885 & 0.115 & 0.043 & 7036 \\
\hline 7 & 62.1 & 16 & 3 & 150.75 & 293 & 1520 & 1453 & 0.606 & 0.394 & 742 & 0.868 & 0.132 & 0.052 & 5624 \\
\hline 7 & 62.1 & 16 & 4 & 152.25 & 314 & 1520 & 1453 & 0.598 & 0.402 & 781 & 0.836 & 0.164 & 0.066 & 4762 \\
\hline 7 & 62.1 & 16 & 5 & 153.75 & 268 & 1520 & 1453 & 0.604 & 0.396 & 677 & 0.892 & 0.108 & 0.043 & 6269 \\
\hline 7 & 62.1 & 16 & 6 & 155.25 & 275 & 1520 & 1453 & 0.584 & 0.416 & 660 & 0.807 & 0.193 & 0.080 & 3422 \\
\hline 7 & 62.1 & 17 & 1 & 156.75 & 310 & 1520 & 1453 & 0.631 & 0.369 & 840 & 0.864 & 0.136 & 0.050 & 6174 \\
\hline 7 & 62.1 & 17 & 2 & 158.25 & 314 & 1517 & 1409 & 0.604 & 0.396 & 792 & 0.864 & 0.136 & 0.054 & 5821 \\
\hline 7 & 62.1 & 17 & 3 & 159.75 & 318 & 1491 & 1460 & 0.604 & 0.396 & 805 & 0.973 & 0.027 & 0.011 & 29815 \\
\hline 7 & 62.1 & 17 & 4 & 161.25 & 318 & 1491 & 1460 & 0.596 & 0.404 & 788 & 0.897 & 0.103 & 0.042 & 7654 \\
\hline 7 & 62.1 & 17 & 5 & 162.75 & 269 & 1491 & 1460 & 0.591 & 0.408 & 658 & 0.930 & 0.070 & 0.029 & 9393 \\
\hline 7 & 62.1 & 17 & 6 & 164.25 & 352 & 1491 & 1460 & 0.586 & 0.414 & 850 & 0.832 & 0.168 & 0.069 & 5060 \\
\hline
\end{tabular}


TABLE 1 - Continued

\begin{tabular}{|c|c|c|c|c|c|c|c|c|c|c|c|c|c|c|}
\hline Leg & Hole & Core & Section & $\begin{array}{l}\text { Depth Below } \\
\text { Sea Floor } \\
\text { (Meters) }\end{array}$ & $\begin{array}{c}\gamma^{\mathrm{a}} \\
\text { Gamma } \\
\text { Radiation }\end{array}$ & $\begin{array}{c}\text { Mean } \\
\text { Water } \\
\text { Control }\end{array}$ & $\begin{array}{c}\text { Mean } \\
\text { Air } \\
\text { Control }\end{array}$ & $\begin{array}{c}\phi \\
\text { Porosity } \\
\text { Fraction }\end{array}$ & $\begin{array}{c}1-\phi \\
\text { Solid } \\
\text { Fraction }\end{array}$ & $\gamma /(1-\phi)$ & $\begin{array}{c}\mathrm{CO}_{3} \\
\text { Weight } \\
\text { Fraction }\end{array}$ & $\begin{array}{l}1-\mathrm{CO}_{3} \\
\text { Weight } \\
\text { Fraction }\end{array}$ & $\begin{array}{c}(1-\phi)\left(1-\mathrm{CO}_{3}\right) \\
\text { Weight } \\
\text { Fraction }\end{array}$ & $\gamma /(1-\phi)\left(1-\mathrm{CO}_{3}\right)$ \\
\hline \multicolumn{15}{|c|}{ Site 62 - Continued } \\
\hline 7 & 62.1 & 18 & 1 & 165.75 & 269 & 1468 & 1421 & 0.556 & 0.444 & 606 & 0.830 & 0.120 & 0.053 & 5052 \\
\hline 7 & 62.1 & 18 & 2 & 167.25 & 263 & 1496 & 1421 & 0.560 & 0.440 & 597 & 0.911 & 0.089 & 0.039 & 6711 \\
\hline 7 & 62.1 & 18 & 3 & 168.75 & 288 & 1496 & 1425 & 0.589 & 0.411 & 702 & 0.940 & 0.060 & 0.025 & 11700 \\
\hline 7 & 62.1 & 18 & 4 & 170.25 & 287 & 1496 & 1425 & 0.566 & 0.434 & 662 & 0.935 & 0.065 & 0.028 & 10132 \\
\hline 7 & 62.1 & 18 & 5 & 171.75 & 227 & 1496 & 1425 & 0.578 & 0.422 & 538 & 0.879 & 0.121 & 0.051 & 4446 \\
\hline 7 & 62.1 & 18 & 6 & 173.25 & 313 & 1496 & 1425 & 0.559 & 0.441 & 710 & 0.888 & 0.112 & 0.049 & 6339 \\
\hline 7 & 62.1 & 19 & 1 & 174.75 & 280 & 1469 & 1473 & 0.619 & 0.381 & 736 & 0.917 & 0.083 & 0.032 & 8865 \\
\hline 7 & 62.1 & 19 & 2 & 176.25 & 242 & 1492 & 1616 & 0.611 & 0.389 & 621 & 0.946 & 0.054 & 0.021 & 11507 \\
\hline 7 & 62.1 & 19 & 3 & 177.75 & 283 & 1492 & 1616 & 0.555 & 0.445 & 637 & 0.960 & 0.040 & 0.018 & 15915 \\
\hline 7 & 62.1 & 19 & 4 & 179.25 & 267 & 1492 & 1616 & 0.583 & 0.437 & 610 & 0.924 & 0.076 & 0.033 & 8026 \\
\hline 7 & 62.1 & 19 & 5 & 180.75 & 272 & 1492 & 1616 & 0.596 & 0.404 & 672 & 0.920 & 0.080 & 0.032 & 8398 \\
\hline 7 & 62.1 & 19 & 6 & 182.25 & 294 & 1492 & 1616 & 0.560 & 0.440 & 669 & 0.620 & 0.380 & 0.167 & 1762 \\
\hline 7 & 62.1 & 20 & 1 & 186.75 & 155 & 1495 & 1452 & 0.590 & 0.410 & 621 & 0.908 & 0.092 & 0.038 & 6749 \\
\hline 7 & 62.1 & 20 & 2 & 188.25 & 256 & 1495 & 1452 & 0.578 & 0.422 & 605 & 0.790 & 0.210 & 0.089 & 2882 \\
\hline 7 & 62.1 & 20 & 3 & 189.75 & 253 & 1495 & 1452 & 0.574 & 0.426 & 594 & 0.931 & 0.069 & 0.029 & 8611 \\
\hline 7 & 62.1 & 20 & 4 & 191.25 & 265 & 1495 & 1452 & 0.561 & 0.439 & 604 & 0.894 & 0.106 & 0.047 & 5694 \\
\hline 7 & 62.1 & 20 & 5 & 192.75 & 236 & 1495 & 1452 & 0.573 & 0.427 & 552 & 0.930 & 0.070 & 0.030 & 7836 \\
\hline 7 & 62.1 & 20 & 6 & 194.25 & 255 & 1495 & 1452 & 0.569 & 0.431 & 592 & 0.929 & 0.071 & 0.031 & 8339 \\
\hline 7 & 62.1 & 21 & 1 & 195.75 & 213 & 1488 & 1443 & 0.587 & 0.413 & 514 & 0.888 & 0.112 & 0.046 & 4593 \\
\hline 7 & 62.1 & 21 & 2 & 197.25 & 266 & 1506 & 1459 & 0.553 & 0.447 & 596 & 0.943 & 0.057 & 0.025 & 10452 \\
\hline 7 & 62.1 & 21 & 3 & 198.75 & 255 & 1506 & 1459 & 0.550 & 0.450 & 567 & $0: 894$ & 0.106 & 0.048 & 5346 \\
\hline 7 & 62.1 & 21 & 4 & 200.25 & 231 & 1506 & 1459 & 0.541 & 0.459 & 502 & 0.833 & 0.167 & 0.077 & 3008 \\
\hline 7 & 62.1 & 21 & 5 & 201.75 & 243 & 1506 & 1459 & 0.540 & 0.460 & 527 & 0.934 & 0.066 & 0.030 & 7985 \\
\hline 7 & 62.1 & 21 & 6 & 203.25 & 173 & 1506 & 1459 & 0.537 & 0.463 & 374 & 0.745 & 0.255 & 0.118 & 1465 \\
\hline 7 & 62.1 & 22 & 1 & 207.75 & 223 & 1506 & 1459 & 0.583 & 0.437 & 510 & 0.922 & 0.078 & 0.034 & 6535 \\
\hline
\end{tabular}


TABLE 1 - Continued

\begin{tabular}{|c|c|c|c|c|c|c|c|c|c|c|c|c|c|c|}
\hline Leg & Hole & Core & Section & $\begin{array}{c}\text { Depth Below } \\
\text { Sea Floor } \\
\text { (Meters) }\end{array}$ & $\begin{array}{c}\gamma^{\mathrm{a}} \\
\text { Gamma } \\
\text { Radiation }\end{array}$ & \begin{tabular}{|c} 
Mean \\
Water \\
Control
\end{tabular} & $\begin{array}{l}\text { Mean } \\
\text { Air } \\
\text { Control }\end{array}$ & $\begin{array}{c}\phi \\
\text { Porosity } \\
\text { Fraction }\end{array}$ & $\begin{array}{c}1-\phi \\
\text { Solid } \\
\text { Fraction }\end{array}$ & $\gamma /(1-\phi)$ & $\begin{array}{c}\mathrm{CO}_{3} \\
\text { Weight } \\
\text { Fraction }\end{array}$ & \begin{tabular}{|c|}
$1-\mathrm{CO}_{3}$ \\
Weight \\
Fraction
\end{tabular} & $\begin{array}{c}(1-\phi)\left(1-\mathrm{CO}_{3}\right) \\
\text { Weight } \\
\text { Fraction }\end{array}$ & $\gamma /(1-\phi)\left(1-\mathrm{CO}_{3}\right)$ \\
\hline \multicolumn{15}{|c|}{ Site $62-$ Continued } \\
\hline 7 & 62.1 & 22 & 2 & 209.25 & 229 & 1489 & 1463 & 0.560 & 0.440 & 520 & 0.926 & 0.074 & 0.033 & 7021 \\
\hline 7 & 62.1 & 22 & 3 & 210.75 & 245 & 1489 & 1463 & 0.535 & 0.465 & 527 & 0.898 & 0.102 & 0.047 & 5263 \\
\hline 7 & 62.1 & 22 & 4 & 212.25 & 224 & 1489 & 1463 & 0.552 & 0.448 & 500 & 0.905 & 0.095 & 0.043 & 5262 \\
\hline 7 & 62.1 & 22 & 5 & 213.75 & 209 & 1489 & 1463 & 0.527 & 0.473 & 442 & 0.921 & 0.079 & 0.037 & 5594 \\
\hline 7 & 62.1 & 22 & 6 & 215.25 & 230 & 1489 & 1463 & 0.574 & 0.426 & 540 & 0.914 & 0.086 & 0.037 & 6283 \\
\hline 7 & 62.1 & 23 & 1 & 216.75 & 234 & 1497 & 1471 & 0.558 & 0.442 & 531 & 0.879 & 0.121 & 0.053 & 4384 \\
\hline 7 & 62.1 & 23 & 2 & 218.25 & 233 & 1503 & 1429 & 0.545 & 0.455 & 513 & 0.752 & 0.248 & 0.113 & 2067 \\
\hline 7 & 62.1 & 23 & 3 & 219.75 & 250 & 1503 & 1429 & 0.542 & 0.458 & 547 & 0.603 & 0.397 & 0.182 & 1377 \\
\hline 7 & 62.1 & 23 & 4 & 221.25 & 244 & 1503 & 1429 & 0.539 & 0.461 & 530 & 0.892 & 0.108 & 0.050 & 4906 \\
\hline 7 & 62.1 & 23 & 5 & 222.75 & 222 & 1503 & 1429 & 0.546 & 0.454 & 489 & 0.696 & 0.304 & 0.138 & 1608 \\
\hline 7 & 62.1 & 23 & 6 & 224.25 & 228 & 1503 & 1429 & 0.532 & 0.468 & 488 & 0.911 & 0.089 & 0.042 & 5479 \\
\hline 7 & 62.1 & 24 & 1 & 225.75 & 220 & 1496 & 1415 & 0.530 & 0.470 & 467 & 0.975 & 0.025 & 0.012 & 18690 \\
\hline 7 & 62.1 & 24 & 2 & 227.25 & 205 & 1446 & 1428 & 0.567 & 0.433 & 474 & 0.940 & 0.060 & 0.026 & 7907 \\
\hline 7 & 62.1 & 24 & 3 & 228.75 & 227 & 1446 & 1428 & 0.549 & 0.451 & 504 & 0.900 & 0.100 & 0.045 & 5042 \\
\hline 7 & 62.1 & 24 & 4 & 230.25 & 243 & 1446 & 1428 & 0.549 & 0.451 & 538 & 0.905 & 0.095 & 0.043 & 5656 \\
\hline 7 & 62.1 & 24 & 5 & 231.75 & 244 & 1446 & 1428 & 0.557 & 0.443 & 550 & 0.691 & 0.309 & 0.137 & 1730 \\
\hline 7 & 62.1 & 24 & 6 & 233.25 & 225 & 1446 & 1428 & 0.548 & 0.452 & 499 & 0.860 & 0.140 & 0.063 & 3554 \\
\hline 7 & 62.1 & 25 & 1 & 234.75 & 248 & 1530 & 1463 & 0.575 & 0.425 & 583 & 0.905 & 0.095 & 0.040 & 6136 \\
\hline 7 & 62.1 & 25 & 2 & 236.25 & 229 & 1501 & 1426 & 0.752 & 0.248 & 923 & 0.871 & 0.129 & 0.032 & 7152 \\
\hline 7 & 62.1 & 25 & 3 & 237.75 & 250 & 1501 & 1426 & 0.594 & 0.406 & 615 & 0.887 & 0.113 & 0.046 & 5444 \\
\hline 7 & 62.1 & 25 & 4 & 239.25 & 254 & 1501 & 1426 & 0.574 & 0.426 & 595 & 0.853 & 0.147 & 0.063 & 4050 \\
\hline 7 & 62.1 & 25 & 5 & 240.75 & 223 & 1501 & 1426 & 0.773 & 0.227 & 984 & 0.873 & 0.127 & 0.029 & 7746 \\
\hline 7 & 62.1 & 26 & 1 & 245.75 & 251 & 1528 & 1468 & 0.569 & 0.431 & 583 & 0.764 & 0.236 & 0.102 & 2459 \\
\hline 7 & 62.1 & 26 & 3 & 248.75 & 256 & 1509 & 1451 & 0.567 & 0.433 & 592 & 0.848 & 0.152 & 0.066 & 3892 \\
\hline 7 & 62.1 & 26 & 4 & 250.25 & 231 & 1509 & 1451 & 0.561 & 0.439 & 526 & 0.843 & 0.157 & 0.069 & 3350 \\
\hline
\end{tabular}


TABLE 1 - Continued

\begin{tabular}{|c|c|c|c|c|c|c|c|c|c|c|c|c|c|c|}
\hline Leg & Hole & Core & Section & $\begin{array}{l}\text { Depth Below } \\
\text { Sea Floor } \\
\text { (Meters) }\end{array}$ & $\begin{array}{c}\gamma^{\mathrm{a}} \\
\text { Gamma } \\
\text { Radiation }\end{array}$ & $\begin{array}{c}\text { Mean } \\
\text { Water } \\
\text { Control } \\
\end{array}$ & \begin{tabular}{|c} 
Mean \\
Water \\
Control \\
\end{tabular} & $\begin{array}{c}\phi \\
\text { Porosity } \\
\text { Fraction }\end{array}$ & \begin{tabular}{|c|}
$1-\phi$ \\
Solid \\
Fraction \\
\end{tabular} & $\gamma /(1-\phi)$ & $\begin{array}{c}\mathrm{CO}_{3} \\
\text { Weight } \\
\text { Fraction }\end{array}$ & $\begin{array}{c}1-\mathrm{CO}_{3} \\
\text { Weight } \\
\text { Fraction }\end{array}$ & $\begin{array}{c}(1-\phi)\left(1-\mathrm{CO}_{3}\right) \\
\text { Weight } \\
\text { Fraction }\end{array}$ & $\gamma /(1-\phi)\left(1-\mathrm{CO}_{3}\right)$ \\
\hline \multicolumn{15}{|c|}{ Site 62 - Continued } \\
\hline 7 & 62.1 & 26 & 5 & 251.75 & 212 & 1509 & 1451 & 0.582 & 0.418 & 506 & 0.931 & 0.069 & 0.029 & 7330 \\
\hline 7 & 62.1 & 26 & 6 & 253.25 & 211 & 1509 & 1451 & 0.605 & 0.395 & 532 & 0.853 & 0.147 & 0.058 & 3622 \\
\hline 7 & 62.1 & 27 & 1 & 254.75 & 204 & 1507 & 1471 & 0.611 & 0.389 & 523 & 0.895 & 0.105 & 0.041 & 4935 \\
\hline 7 & 62.1 & 27 & 2 & 256.25 & 216 & 1483 & 1445 & 0.588 & 0.412 & 525 & 0.860 & 0.140 & 0.058 & 3748 \\
\hline 7 & 62.1 & 27 & 3 & 257.75 & 232 & 1483 & 1445 & 0.577 & 0.423 & 549 & 0.743 & 0.257 & 0.109 & 2135 \\
\hline 7 & 62.1 & 27 & 4 & 259.25 & 206 & 1483 & 1445 & 0.554 & 0.446 & 462 & 0.880 & 0.120 & 0.054 & 3849 \\
\hline 7 & 62.1 & 27 & 5 & 260.75 & 224 & 1483 & 1445 & 0.556 & 0.444 & 503 & 0.896 & 0.104 & 0.046 & 4839 \\
\hline 7 & 62.1 & 28 & 1 & 263.75 & 210 & 1530 & 1450 & 0.608 & 0.392 & 535 & 0.859 & 0.141 & 0.055 & 3793 \\
\hline 7 & 62.1 & 28 & 2 & 265.25 & 214 & 1512 & 1481 & 0.589 & 0.411 & 521 & 0.868 & 0.132 & 0.054 & 3948 \\
\hline 7 & 62.1 & 28 & 3 & 266.75 & 222 & 1512 & 1481 & 0.567 & 0.433 & 512 & 0.817 & 0.183 & 0.079 & 2799 \\
\hline 7 & 62.1 & 28 & 4 & 268.25 & 219 & 1512 & 1481 & 0.599 & 0.401 & 545 & 0.743 & 0.257 & 0.103 & 2119 \\
\hline 7 & 62.1 & 28 & 5 & 269.75 & 203 & 1512 & 1481 & 0.616 & 0.384 & 529 & 0.804 & 0.196 & 0.075 & 2701 \\
\hline 7 & 62.1 & 28 & 6 & 271.25 & 210 & 1512 & 1481 & 0.608 & 0.392 & 535 & 0.519 & 0.481 & 0.188 & 1113 \\
\hline 7 & 62.1 & 29 & 1 & 271.75 & 217 & 1500 & 1463 & 0.603 & 0.397 & 547 & 0.920 & 0.080 & 0.032 & 6838 \\
\hline 7 & 62.1 & 29 & 2 & 273.25 & 197 & 1470 & 1437 & 0.605 & 0.395 & 500 & 0.864 & 0.136 & 0.054 & 3677 \\
\hline 7 & 62.1 & 29 & 3 & 274.75 & 219 & 1470 & 1437 & 0.593 & 0.407 & 538 & 0.845 & 0.155 & 0.063 & 3471 \\
\hline 7 & 62.1 & 29 & 4 & 276.25 & 205 & 1470 & 1437 & 0.609 & 0.391 & 525 & 0.844 & 0.156 & 0.061 & 3363 \\
\hline 7 & 62.1 & 29 & 5 & 277.75 & 193 & 1470 & 1437 & 0.595 & 0.405 & 476 & 0.864 & 0.136 & 0.055 & 3502 \\
\hline 7 & 62.1 & 29 & 6 & 279.25 & 205 & 1470 & 1437 & 0.599 & 0.401 & 512 & 0.851 & 0.149 & 0.060 & 3435 \\
\hline 7 & 62.1 & 30 & 1 & 281.75 & 215 & 1521 & 1458 & 0.578 & 0.422 & 509 & 0.875 & 0.125 & 0.053 & 4074 \\
\hline 7 & 62.1 & 30 & 2 & 283.25 & 181 & 1481 & 1424 & 0.586 & 0.414 & 437 & 0.875 & 0.125 & 0.052 & 3494 \\
\hline 7 & 62.1 & 30 & 3 & 284.75 & 217 & 1481 & 1424 & 0.567 & 0.433 & 500 & 0.876 & 0.124 & 0.054 & 4035 \\
\hline 7 & 62.1 & 30 & 4 & 286.25 & 191 & 1481 & 1424 & 0.574 & 0.426 & 448 & 0.908 & 0.092 & 0.039 & 4867 \\
\hline 7 & 62.1 & 30 & 5 & 287.75 & 200 & 1481 & 1424 & 0.576 & 0.424 & 471 & 0.891 & 0.109 & 0.046 & 4317 \\
\hline 7 & 62.1 & 30 & 6 & 289.25 & 184 & 1481 & 1424 & 0.592 & 0.408 & 450 & 0.407 & 0.593 & 0.242 & 759 \\
\hline
\end{tabular}


TABLE 1 - Continued

\begin{tabular}{|c|c|c|c|c|c|c|c|c|c|c|c|c|c|c|}
\hline Leg & Hole & Core & Section & $\begin{array}{c}\text { Depth Below } \\
\text { Sea Floor } \\
\text { (Meters) }\end{array}$ & $\begin{array}{c}\gamma^{\mathrm{a}} \\
\text { Gamma } \\
\text { Radiation }\end{array}$ & $\begin{array}{c}\text { Mean } \\
\text { Water } \\
\text { Control }\end{array}$ & $\begin{array}{c}\text { Mean } \\
\text { Air } \\
\text { Control }\end{array}$ & $\begin{array}{c}\phi \\
\text { Porosity } \\
\text { Fraction }\end{array}$ & $\begin{array}{c}1-\phi \\
\text { Solid } \\
\text { Fraction }\end{array}$ & $\gamma /(1-\phi)$ & $\begin{array}{c}\mathrm{CO}_{3} \\
\text { Weight } \\
\text { Fraction }\end{array}$ & $\begin{array}{c}1-\mathrm{CO}_{3} \\
\text { Weight } \\
\text { Fraction }\end{array}$ & $\begin{array}{c}(1-\phi)\left(1-\mathrm{CO}_{3}\right) \\
\text { Weight } \\
\text { Fraction }\end{array}$ & $\gamma /(1-\phi)\left(1-\mathrm{CO}_{3}\right)$ \\
\hline \multicolumn{3}{|c|}{ Site 62 - Continued } & & & & & & & & & & & & \\
\hline 7 & 62.1 & 31 & 1 & 291.75 & 207 & 1506 & 1472 & 0.558 & 0.442 & 469 & 0.876 & 0.124 & 0.055 & 3782 \\
\hline 7 & 62.1 & 31 & 2 & 293.25 & 211 & 1507 & 1476 & 0.567 & 0.433 & 487 & 0.858 & 0.142 & 0.061 & 3432 \\
\hline 7 & 62.1 & 31 & 3 & 294.75 & 203 & 1507 & 1476 & 0.551 & 0.449 & 452 & 0.870 & 0.130 & 0.058 & 3480 \\
\hline 7 & 62.1 & 31 & 4 & 296.25 & 199 & 1507 & 1476 & 0.546 & 0.454 & 439 & 0.860 & 0.140 & 0.064 & 3132 \\
\hline 7 & 62.1 & 31 & 5 & 297.75 & 177 & 1507 & 1476 & 0.599 & 0.401 & 441 & 0.881 & 0.119 & 0.048 & 3708 \\
\hline 7 & 62.1 & 31 & 6 & 299.25 & 204 & 1507 & 1476 & 0.551 & 0.449 & 454 & 0.895 & 0.105 & 0.047 & 4326 \\
\hline 7 & 62.1 & 32 & 1 & 301.75 & 201 & 1481 & 1437 & 0.584 & 0.416 & 484 & 0.893 & 0.107 & 0.045 & 4519 \\
\hline 7 & 62.1 & 32 & 2 & 303.25 & 175 & 1494 & 1474 & 0.568 & 0.432 & 406 & 0.906 & 0.094 & 0.041 & 4319 \\
\hline 7 & 62.1 & 32 & 3 & 304.75 & 213 & 1494 & 1474 & 0.576 & 0.424 & 502 & 0.930 & 0.070 & 0.030 & 7174 \\
\hline 7 & 62.1 & 32 & 4 & 306.25 & 247 & 1494 & 1474 & 0.581 & 0.419 & 589 & 0.743 & 0.257 & 0.108 & 2290 \\
\hline 7 & 62.1 & 32 & 5 & 307.75 & 210 & 1494 & 1474 & 0.581 & 0.419 & 502 & 0.893 & 0.107 & 0.045 & 4689 \\
\hline 7 & 62.1 & 32 & 6 & 309.25 & 237 & 1494 & 1474 & 0.581 & 0.419 & 566 & 0.866 & 0.134 & 0.056 & 4223 \\
\hline 7 & 62.1 & 33 & 2 & 312.25 & 210 & 1474 & 1432 & 0.569 & 0.431 & 489 & 0.836 & 0.164 & 0.071 & 2980 \\
\hline 7 & 62.1 & 33 & 3 & 313.75 & 228 & 1556 & 1469 & 0.559 & 0.441 & 518 & 0.808 & 0.192 & 0.085 & 2699 \\
\hline 7 & 62.1 & 34 & 1 & 320.75 & 296 & 1476 & 1455 & 0.579 & 0.421 & 704 & 0.813 & 0.187 & 0.079 & 3763 \\
\hline 7 & 62.1 & 34 & 2 & 322.25 & 267 & 1490 & 1450 & 0.536 & 0.464 & 575 & 0.851 & 0.149 & 0.069 & 3859 \\
\hline 7 & 62.1 & 34 & 3 & 323.75 & 316 & 1490 & 1450 & 0.545 & 0.455 & 695 & 0.776 & 0.224 & 0.102 & 3101 \\
\hline 7 & 62.1 & 34 & 4 & 325.25 & 288 & 1490 & 1450 & 0.542 & 0.458 & 628 & 0.827 & 0.173 & 0.079 & 3630 \\
\hline 7 & 62.1 & 34 & 5 & 326.75 & 293 & 1490 & 1450 & 0.519 & 0.481 & 609 & 0.836 & 0.144 & 0.069 & 4226 \\
\hline 7 & 62.1 & 34 & 6 & 328.25 & 248 & 1490 & 1450 & 0.520 & 0.480 & 516 & 0.869 & 0.131 & 0.063 & 3937 \\
\hline 7 & 62.1 & 35 & 1 & 327.75 & 244 & 1505 & 1460 & 0.592 & 0.408 & 600 & 0.775 & 0.225 & 0.092 & 2665 \\
\hline 7 & 62.1 & 35 & 2 & 329.25 & 214 & 1489 & 1463 & 0.591 & 0.409 & 523 & 0.833 & 0.167 & 0.068 & 3133 \\
\hline 7 & 62.1 & 35 & 3 & 330.75 & 253 & 1489 & 1463 & 0.575 & 0.425 & 594 & 0.846 & 0.154 & 0.065 & 3858 \\
\hline 7 & 62.1 & 35 & 4 & 332.25 & 266 & 1489 & 1463 & 0.572 & 0.428 & 620 & 0.836 & 0.164 & 0.070 & 3735 \\
\hline 7 & 62.1 & 35 & 5 & 333.75 & 240 & 1489 & 1463 & 0.583 & 0.417 & 577 & 0.800 & 0.200 & 0.083 & 2885 \\
\hline
\end{tabular}


TABLE 1 - Continued

\begin{tabular}{|c|c|c|c|c|c|c|c|c|c|c|c|c|c|c|}
\hline Leg & Hole & Core & Section & $\begin{array}{c}\text { Depth Below } \\
\text { Sea Floor } \\
\text { (Meters) }\end{array}$ & $\begin{array}{c}\gamma^{\mathrm{a}} \\
\text { Gamma } \\
\text { Radiation }\end{array}$ & $\begin{array}{c}\text { Mean } \\
\text { Water } \\
\text { Control }\end{array}$ & $\begin{array}{c}\text { Mean } \\
\text { Air } \\
\text { Control }\end{array}$ & $\begin{array}{c}\phi \\
\text { Porosity } \\
\text { Fraction }\end{array}$ & $\begin{array}{c}1-\phi \\
\text { Solid } \\
\text { Fraction }\end{array}$ & $\gamma /(1-\phi)$ & \begin{tabular}{c|}
$\mathrm{CO}_{3}$ \\
Weight \\
Fraction
\end{tabular} & $\begin{array}{c}1-\mathrm{CO}_{3} \\
\text { Weight } \\
\text { Fraction }\end{array}$ & $\begin{array}{c}(1-\phi)\left(1-\mathrm{CO}_{3}\right) \\
\text { Weight } \\
\text { Fraction }\end{array}$ & $\gamma /(1-\phi)\left(1-\mathrm{CO}_{3}\right)$ \\
\hline \multicolumn{15}{|c|}{ Site 62 - Continued } \\
\hline 7 & 62.1 & 35 & 6 & 335.25 & 253 & 1489 & 1463 & 0.570 & 0.430 & 588 & 0.846 & 0.154 & 0.066 & 3817 \\
\hline 7 & 62.1 & 36 & 2 & 338.25 & 219 & 1505 & 1432 & 0.592 & 0.408 & 538 & 0.848 & 0.152 & 0.062 & 3541 \\
\hline 7 & 62.1 & 36 & 3 & 339.75 & 232 & 1505 & 1432 & 0.571 & 0.429 & 541 & 0.833 & 0.167 & 0.072 & 3237 \\
\hline 7 & 62.1 & 36 & 4 & 341.25 & 241 & 1505 & 1432 & 0.537 & 0.463 & 520 & 0.883 & 0.117 & 0.054 & 4448 \\
\hline 7 & 62.1 & 36 & 5 & 342.75 & 263 & 1505 & 1432 & 0.505 & 0.435 & 604 & 0.855 & 0.145 & 0.063 & 4165 \\
\hline 7 & 62.1 & 37 & 2 & 346.25 & 250 & 1498 & 1442 & 0.566 & 0.434 & 576 & 0.836 & 0.164 & 0.071 & 3513 \\
\hline \multicolumn{15}{|l|}{ Site 63} \\
\hline 7 & 63 & 1 & 1 & .75 & 1582 & 1455 & 1424 & 0.767 & 0.233 & 6776 & 0.036 & 0.964 & 0.225 & 7029 \\
\hline 7 & 63 & 1 & 2 & 2.25 & 906 & 1455 & 1424 & 0.770 & 0.230 & 3938 & 0.080 & 0.920 & 0.212 & 4230 \\
\hline 7 & 63 & 1 & 3 & 3.75 & 721 & 1455 & 1424 & 0.748 & 0.252 & 2864 & 0.106 & 0.894 & 0.225 & 3203 \\
\hline 7 & 63 & 1 & 4 & 5.25 & 614 & 1455 & 1424 & 0.792 & 0.208 & 2952 & 0.120 & 0.880 & 0.183 & 3354 \\
\hline 7 & 63 & 2 & 2 & 63.25 & 298 & 1483 & 1417 & 0.642 & 0.358 & 832 & 0.670 & 0.330 & 0.118 & 2522 \\
\hline 7 & 63 & 2 & 3 & 64.75 & 216 & 1483 & 1417 & 0.608 & 0.392 & 549 & 0.810 & 0.190 & 0.075 & 2891 \\
\hline 7 & 63 & 2 & 4 & 66.25 & 266 & 1483 & 1417 & 0.642 & 0.358 & 744 & 0.796 & 0.204 & 0.073 & 3646 \\
\hline 7 & 63 & 2 & 5 & 67.75 & 260 & 1483 & 1417 & 0.614 & 0.386 & 675 & 0.810 & 0.190 & 0.073 & 3552 \\
\hline 7 & 63 & 2 & 6 & 69.25 & 268 & 1483 & 1417 & 0.599 & 0.401 & 668 & 0.610 & 0.390 & 0.156 & 1713 \\
\hline 7 & 63 & 3 & 2 & 139.25 & 258 & 1477 & 1437 & 0.589 & 0.411 & 628 & 0.640 & 0.360 & 0.148 & 1744 \\
\hline 7 & 63 & 3 & 3 & 140.75 & 262 & 1477 & 1437 & 0.581 & 0.419 & 627 & 0.791 & 0.209 & 0.088 & 2998 \\
\hline 7 & 63 & 3 & 4 & 142.25 & 323 & 1477 & 1437 & 0.593 & 0.407 & 793 & 0.566 & 0.434 & 0.177 & 1827 \\
\hline 7 & 63 & 4 & 1 & 230.75 & 193 & 1493 & 1450 & 0.543 & 0.457 & 422 & 0.764 & 0.236 & 0.108 & 1787 \\
\hline 7 & 63 & 4 & 2 & 232.25 & 194 & 1493 & 1450 & 0.494 & 0.506 & 383 & 0.778 & 0.222 & 0.112 & 1724 \\
\hline 7 & 63 & 5 & 2 & 354.25 & 181 & 1472 & 1439 & 0.475 & 0.525 & 344 & 0.806 & 0.194 & 0.102 & 1773 \\
\hline 7 & 63 & 6 & 2 & 458.75 & 172 & 1546 & 1431 & 0.457 & 0.543 & 316 & 0.886 & 0.114 & 0.062 & 2772 \\
\hline 7 & 63 & 6 & 2 & 460.25 & 170 & 1546 & 1431 & 0.479 & 0.521 & 326 & 0.906 & 0.094 & 0.049 & 3470 \\
\hline
\end{tabular}


TABLE 1 - Continued

\begin{tabular}{|c|c|c|c|c|c|c|c|c|c|c|c|c|c|c|}
\hline Leg & Hole & Core & Section & $\begin{array}{l}\text { Depth Below } \\
\text { Sea Floor } \\
\text { (Meters) }\end{array}$ & $\begin{array}{c}\gamma^{\mathrm{a}} \\
\text { Gamma } \\
\text { Radiation }\end{array}$ & $\begin{array}{c}\text { Mean } \\
\text { Water } \\
\text { Control }\end{array}$ & \begin{tabular}{|c} 
Mean \\
Air \\
Control
\end{tabular} & $\begin{array}{c}\phi \\
\text { Porosity } \\
\text { Fraction } \\
\end{array}$ & $\begin{array}{c}1-\phi \\
\text { Solid } \\
\text { Fraction }\end{array}$ & $\gamma /(1-\phi)$ & $\begin{array}{c}\mathrm{CO}_{3} \\
\text { Weight } \\
\text { Fraction }\end{array}$ & $\begin{array}{c}1-\mathrm{CO}_{3} \\
\text { Weight } \\
\text { Fraction }\end{array}$ & $\begin{array}{c}(1-\phi)\left(1-\mathrm{CO}_{3}\right) \\
\text { Weight } \\
\text { Fraction }\end{array}$ & $\gamma /(1-\phi)\left(1-\mathrm{CO}_{3}\right)$ \\
\hline \multicolumn{15}{|c|}{ Site 63 - Continued } \\
\hline 7 & 63 & 6 & 3 & 461.75 & 181 & 1546 & 1431 & 0.446 & 0.554 & 327 & 0.900 & 0.100 & 0.055 & 3272 \\
\hline 7 & 63 & 6 & 4 & 463.25 & 176 & 1546 & 1431 & 0.453 & 0.547 & 322 & 0.941 & 0.059 & 0.032 & 5449 \\
\hline 7 & 63 & 6 & 5 & 464.75 & 193 & 1546 & 1431 & 0.436 & 0.564 & 342 & 0.900 & 0.100 & 0.056 & 3415 \\
\hline 7 & 63 & 6 & 6 & 466.25 & 149 & 1546 & 1431 & 0.445 & 0.555 & 269 & 0.978 & 0.022 & 0.012 & 12238 \\
\hline 7 & 63 & 7 & 1 & 534.75 & 126 & 1508 & 1431 & 0.440 & 0.560 & 225 & 0.872 & 0.128 & 0.072 & 1757 \\
\hline 7 & 63 & 7 & 2 & 536.25 & 127 & 1508 & 1431 & 0.442 & 0.558 & 228 & 0.892 & 0.108 & 0.060 & 2113 \\
\hline 7 & 63 & 7 & 3 & 537.75 & 146 & 1508 & 1431 & 0.417 & 0.583 & 251 & 0.863 & 0.137 & 0.080 & 1830 \\
\hline 7 & 63 & 7 & 4 & 539.25 & 129 & 1508 & 1431 & 0.413 & 0.587 & 220 & 0.875 & 0.125 & 0.073 & 1759 \\
\hline 7 & 63 & 7 & 5 & 540.75 & 132 & 1508 & 1431 & 0.399 & 0.601 & 220 & 0.850 & 0.120 & 0.072 & 1837 \\
\hline 7 & 63 & 7 & 6 & 542.25 & 125 & 1508 & 1431 & 0.393 & 0.607 & 206 & 0.940 & 0.060 & 0.036 & 3433 \\
\hline 7 & 63 & 8 & 2 & 545.25 & 135 & 1488 & 1414 & 0.438 & 0.562 & 239 & 0.891 & 0.109 & 0.061 & 2197 \\
\hline 7 & 63 & 8 & 3 & 546.75 & 137 & 1488 & 1414 & 0.424 & 0.576 & 238 & 0.850 & 0.150 & 0.086 & 1537 \\
\hline 7 & 63 & 9 & 1 & 553.75 & 124 & 1463 & 1450 & 0.388 & 0.612 & 202 & 0.913 & 0.087 & 0.053 & 2324 \\
\hline 7 & 63 & 9 & 2 & 555.25 & 139 & 1463 & 1450 & 0.385 & 0.615 & 225 & 0.916 & 0.084 & 0.052 & 2631 \\
\hline 7 & 63 & 9 & 3 & 556.75 & 126 & 1463 & 1450 & 0.359 & 0.641 & 196 & 0.918 & 0.082 & 0.053 & 2395 \\
\hline 7 & 63 & 9 & 4 & 558.25 & 151 & 1463 & 1450 & 0.353 & 0.647 & 233 & 0.891 & 0.109 & 0.071 & 2142 \\
\hline 7 & 63.1 & 1 & 1 & 3.75 & 566 & 1452 & 1420 & 0.819 & 0.181 & 3126 & 0.227 & 0.773 & 0.140 & 4043 \\
\hline 7 & 63.1 & 1 & 2 & 5.25 & 637 & 1452 & 1420 & 0.812 & 0.188 & 3396 & 0.122 & 0.878 & 0.165 & 3868 \\
\hline 6 & 63.1 & 1 & 3 & 6.75 & 568 & 1452 & 1420 & 0.815 & 0.185 & 3065 & 0.215 & 0.785 & 0.146 & 3905 \\
\hline 7 & 63.1 & 1 & 4 & 8.25 & 515 & 1452 & 1420 & 0.862 & 0.138 & 3730 & 0.110 & 0.890 & 0.123 & 4191 \\
\hline 7 & 63.1 & 3 & 1 & 22.75 & 446 & 1448 & 1450 & 0.795 & 0.205 & 2176 & 0.305 & 0.695 & 0.142 & 3131 \\
\hline 7 & 63.1 & 3 & 2 & 24.25 & 707 & 1448 & 1450 & 0.776 & 0.224 & 3156 & 0.297 & 0.703 & 0.157 & 4490 \\
\hline 7 & 63.1 & 5 & 1 & 101.75 & 222 & 1496 & 1426 & 0.691 & 0.309 & 718 & 0.624 & 0.376 & 0.116 & 1909 \\
\hline 7 & 63.1 & 5 & 2 & 103.25 & 313 & 1496 & 1426 & 0.652 & 0.348 & 899 & 0.630 & 0.370 & 0.129 & 2430 \\
\hline 7 & 63.1 & 5 & 3 & 104.75 & 284 & 1496 & 1426 & 0.647 & 0.353 & 806 & 0.712 & 0.288 & 0.102 & 2798 \\
\hline
\end{tabular}


TABLE 1 - Continued

\begin{tabular}{|c|c|c|c|c|c|c|c|c|c|c|c|c|c|c|}
\hline Leg & Hole & Core & Section & $\begin{array}{l}\text { Depth Below } \\
\text { Sea Floor } \\
\text { (Meters) }\end{array}$ & $\begin{array}{c}\gamma^{\mathrm{a}} \\
\text { Gamma } \\
\text { Radiation }\end{array}$ & $\begin{array}{c}\text { Mean } \\
\text { Water } \\
\text { Control }\end{array}$ & $\begin{array}{c}\text { Mean } \\
\text { Air } \\
\text { Control }\end{array}$ & $\begin{array}{c}\phi \\
\text { Porosity } \\
\text { Fraction }\end{array}$ & $\begin{array}{c}1-\phi \\
\text { Solid } \\
\text { Fraction }\end{array}$ & $\gamma /(1-\phi)$ & $\begin{array}{c}\mathrm{CO}_{3} \\
\text { Weight } \\
\text { Fractionn }\end{array}$ & $\begin{array}{c}1-\mathrm{CO}_{3} \\
\text { Weight } \\
\text { Fraction }\end{array}$ & $\begin{array}{c}(1-\phi)\left(1-\mathrm{CO}_{3}\right) \\
\text { Weight } \\
\text { Fraction }\end{array}$ & $\gamma /(1-\phi)\left(1-\mathrm{CO}_{3}\right)$ \\
\hline \multicolumn{15}{|c|}{ Site 63 - Continued } \\
\hline 7 & 63.1 & 5 & 4 & 106.25 & 323 & 1496 & 1426 & 0.676 & 0.324 & 996 & 0.453 & 0.547 & 0.177 & 1821 \\
\hline 7 & 63.1 & 6 & 1 & 101.75 & 286 & 1473 & 1436 & 0.718 & 0.282 & 1013 & 0.532 & 0.468 & 0.132 & 2165 \\
\hline 7 & 63.1 & 6 & 2 & 112.25 & 429 & 1473 & 1436 & 0.707 & 0.293 & 1462 & 0.364 & 0.636 & 0.187 & 2298 \\
\hline 7 & 63.1 & 6 & 3 & 113.75 & 379 & 1473 & 1436 & 0.703 & 0.297 & 1275 & 0.455 & 0.545 & 0.162 & 2340 \\
\hline 7 & 63.1 & 6 & 4 & 115.25 & 402 & 1473 & 1436 & 0.693 & 0.307 & 1309 & 0.533 & 0.467 & 0.143 & 2803 \\
\hline 7 & 63.1 & 6 & 5 & 116.75 & 403 & 1473 & 1436 & 0.674 & 0.326 & 1234 & 0.445 & 0.555 & 0.181 & 2224 \\
\hline 7 & 63.1 & 6 & 6 & 118.25 & 362 & 1473 & 1436 & 0.844 & 0.356 & 1017 & 0.548 & 0.452 & 0.161 & 2249 \\
\hline 7 & 63.1 & 7 & 2 & 121.25 & 336 & 1467 & 1429 & 0.703 & 0.297 & 1131 & 0.710 & 0.290 & 0.086 & 3902 \\
\hline 7 & 63.1 & 7 & 3 & 122.75 & 364 & 1467 & 1429 & 0.699 & 0.301 & 1208 & 0.565 & 0.435 & 0.131 & 2777 \\
\hline 7 & 63.1 & 7 & 4 & 124.25 & 385 & 1467 & 1429 & 0.700 & 0.300 & 1283 & 0.449 & 0.551 & 0.165 & 2328 \\
\hline 7 & 63.1 & 8 & 3 & 132.75 & 257 & 1483 & 1446 & 0.896 & 0.104 & 2477 & 0.616 & 0.384 & 0.040 & 6449 \\
\hline 7 & 63.1 & 8 & 4 & 134.25 & 293 & 1483 & 1446 & 0.792 & 0.208 & 1409 & 0.628 & 0.372 & 0.077 & 3788 \\
\hline 7 & 63.1 & 8 & 5 & 135.75 & 316 & 1483 & 1446 & 0.768 & 0.232 & 1361 & 0.593 & 0.407 & 0.095 & 3343 \\
\hline 7 & 63.1 & 9 & 1 & 138.75 & 277 & 1479 & 1431 & 0.848 & 0.352 & 785 & 0.736 & 0.264 & 0.093 & 2974 \\
\hline 7 & 63.1 & 9 & 2 & 140.25 & 299 & 1479 & 1431 & 0.614 & 0.386 & 775 & 0.686 & 0.314 & 0.121 & 2468 \\
\hline 7 & 63.1 & 9 & 3 & 141.75 & 317 & 1479 & 1431 & 0.621 & 0.379 & 837 & 0.776 & 0.224 & 0.085 & 3738 \\
\hline 7 & 63.1 & 9 & 4 & 143.25 & 236 & 1479 & 1431 & 0.569 & 0.431 & 547 & 0.713 & 0.287 & 0.124 & 1905 \\
\hline 7 & 63.1 & 9 & 5 & 144.75 & 281 & 1479 & 1431 & 0.601 & 0.399 & 705 & 0.635 & 0.365 & 0.146 & 1930 \\
\hline 7 & 63.1 & 9 & 6 & 146.25 & 244 & 1479 & 1431 & 0.583 & 0.417 & 583 & 0.811 & 0.189 & 0.079 & 3087 \\
\hline 7 & 63.1 & 10 & 1 & 148.75 & 281 & 1475 & 1422 & 0.596 & 0.404 & 695 & 0.704 & 0.296 & 0.120 & 2348 \\
\hline 7 & 63.1 & 10 & 2 & 150.25 & 257 & 1475 & 1422 & 0.586 & 0.414 & 621 & 0.533 & 0.467 & 0.193 & 1329 \\
\hline 7 & 63.1 & 10 & 3 & 151.75 & 317 & 1475 & 1422 & 0.617 & 0.383 & 827 & 0.717 & 0.283 & 0.108 & 2924 \\
\hline 7 & 63.1 & 10 & 4 & 153.25 & 251 & 1475 & 1422 & 0.570 & 0.430 & 583 & 0.652 & 0.348 & 0.150 & 1676 \\
\hline 7 & 63.1 & 10 & 5 & 154.75 & 249 & 1475 & 1422 & 0.566 & 0.434 & 573 & 0.796 & 0.204 & 0.088 & 2808 \\
\hline 7 & 63.1 & 11 & 1 & 155.75 & 262 & 1477 & 1424 & 0.596 & 0.404 & 649 & 0.821 & 0.179 & 0.072 & 3624 \\
\hline
\end{tabular}


TABLE 1 - Continued

\begin{tabular}{|c|c|c|c|c|c|c|c|c|c|c|c|c|c|c|}
\hline Leg & Hole & Core & Section & $\begin{array}{c}\text { Depth Below } \\
\text { Sea Floor } \\
\text { (Meters) }\end{array}$ & $\begin{array}{c}\gamma^{\mathrm{a}} \\
\text { Gamma } \\
\text { Radiation }\end{array}$ & $\begin{array}{l}\text { Mean } \\
\text { Water } \\
\text { Control }\end{array}$ & $\begin{array}{c}\text { Mean } \\
\text { Air } \\
\text { Control }\end{array}$ & $\begin{array}{c}\phi \\
\text { Porosity } \\
\text { Fraction }\end{array}$ & $\begin{array}{c}1-\phi \\
\text { Solid } \\
\text { Fraction }\end{array}$ & $\gamma /(1-\phi)$ & $\begin{array}{c}\mathrm{CO}_{3} \\
\text { Weight } \\
\text { Fraction }\end{array}$ & $\begin{array}{l}1-\mathrm{CO}_{3} \\
\text { Weight } \\
\text { Fraction }\end{array}$ & $\begin{array}{c}(1-\phi)\left(1-\mathrm{CO}_{3}\right) \\
\text { Weight } \\
\text { Fraction }\end{array}$ & $\gamma /(1-\phi)\left(1-\mathrm{CO}_{3}\right)$ \\
\hline \multicolumn{15}{|c|}{ Site $63-$ Continued } \\
\hline 7 & 63.1 & 11 & 2 & 157.25 & 287 & 1477 & 1424 & 0.625 & 0.375 & 763 & 0.656 & 0.344 & 0.129 & 2219 \\
\hline 7 & 63.1 & 11 & 3 & 158.75 & 247 & 1477 & 1424 & 0.582 & 0.418 & 591 & 0.706 & 0.294 & 0.123 & 2011 \\
\hline 7 & 63.1 & 11 & 4 & 160.25 & 214 & 1477 & 1424 & 0.563 & 0.437 & 490 & 0.780 & 0.220 & 0.096 & 2226 \\
\hline 7 & 63.1 & 11 & 6 & 163.25 & 267 & 1477 & 1424 & 0.544 & 0.456 & 584 & 0.706 & 0.294 & 0.134 & 1987 \\
\hline 7 & 63.1 & 12 & 2 & 167.25 & 243 & 1515 & 1459 & 0.541 & 0.459 & 530 & 0.821 & 0.179 & 0.082 & 2960 \\
\hline 7 & 63.1 & 13 & 1 & 174.75 & 235 & 1484 & 1428 & 0.572 & 0.428 & 549 & 0.765 & 0.235 & 0.100 & 2335 \\
\hline 7 & 63.1 & 13 & 2 & 176.25 & 288 & 1484 & 1428 & 0.609 & 0.391 & 737 & 0.739 & 0.261 & 0.102 & 2823 \\
\hline 7 & 63.1 & 13 & 3 & 177.75 & 275 & 1484 & 1428 & 0.557 & 0.443 & 621 & 0.780 & 0.220 & 0.098 & 2822 \\
\hline 7 & 63.1 & 13 & 4 & 179.25 & 335 & 1484 & 1428 & 0.633 & 0.367 & 915 & 0.608 & 0.392 & 0.144 & 2333 \\
\hline 7 & 63.1 & 14 & 3 & 187.75 & 301 & 1549 & 1437 & 0.586 & 0.414 & 726 & 0.738 & 0.262 & 0.108 & 2771 \\
\hline 7 & 63.1 & 14 & 4 & 189.25 & 241 & 1549 & 1437 & 0.570 & 0.430 & 561 & 0.581 & 0.419 & 0.180 & 1339 \\
\hline 7 & 63.1 & 14 & 5 & 190.75 & 213 & 1549 & 1437 & 0.545 & 0.455 & 469 & 0.832 & 0.168 & 0.076 & 2793 \\
\hline 7 & 63.2 & 1 & 3 & 14.75 & 531 & 1505 & 1437 & 0.794 & 0.206 & 2582 & 0.269 & 0.731 & 0.150 & 3532 \\
\hline 7 & 63.2 & 1 & 4 & 16.25 & 586 & 1505 & 1437 & 0.823 & 0.177 & 3313 & 0.178 & 0.822 & 0.145 & 4030 \\
\hline 7 & 63.2 & 2 & 1 & 20.75 & 526 & 1499 & 1461 & 0.801 & 0.199 & 2647 & 0.059 & 0.941 & 0.187 & 2813 \\
\hline 7 & 63.2 & 2 & 2 & 22.25 & 531 & 1499 & 1461 & 0.825 & 0.175 & 3040 & 0.278 & 0.722 & 0.126 & 4210 \\
\hline 7 & 63.2 & 2 & 3 & 23.75 & 543 & 1499 & 1461 & 0.838 & 0.162 & 3346 & 0.173 & 0.827 & 0.134 & 4046 \\
\hline 7 & 63.2 & 2 & 4 & 25.25 & 522 & 1499 & 1461 & 0.839 & 0.161 & 3234 & 0.211 & 0.789 & 0.127 & 4099 \\
\hline 7 & 63.2 & 2 & 6 & 28.25 & 498 & 1499 & 1461 & 0.884 & 0.116 & 4279 & 0.170 & 0.830 & 0.097 & 5155 \\
\hline
\end{tabular}


TABLE 1 - Continued

\begin{tabular}{|c|c|c|c|c|c|c|c|c|c|c|c|c|c|c|}
\hline Leg & Hole & Core & Section & $\begin{array}{c}\text { Depth Below } \\
\text { Sea Floor } \\
\text { (Meters) }\end{array}$ & $\begin{array}{c}\gamma^{\mathrm{a}} \\
\text { Gamma } \\
\text { Radiation }\end{array}$ & $\begin{array}{c}\text { Mean } \\
\text { Water } \\
\text { Control }\end{array}$ & $\begin{array}{c}\text { Mean } \\
\text { Air } \\
\text { Control }\end{array}$ & $\begin{array}{c}\phi \\
\text { Porosity } \\
\text { Fraction }\end{array}$ & $\begin{array}{c}1-\phi \\
\text { Solid } \\
\text { Fraction }\end{array}$ & $\gamma /(1-\phi)$ & $\begin{array}{c}\mathrm{CO}_{3} \\
\text { Weight } \\
\text { Fraction }\end{array}$ & $\begin{array}{c}1-\mathrm{CO}_{3} \\
\text { Weight } \\
\text { Fraction }\end{array}$ & $\begin{array}{c}(1-\phi)\left(1-\mathrm{CO}_{3}\right) \\
\text { Weight } \\
\text { Fraction }\end{array}$ & $\gamma /(1-\phi)\left(1-\mathrm{CO}_{3}\right)$ \\
\hline \multicolumn{15}{|c|}{ Site 63 - Continued } \\
\hline 7 & 63.2 & 3 & 2 & 32.25 & 515 & 1518 & 1452 & 0.783 & 0.217 & 2372 & 0.141 & 0.859 & 0.186 & 2762 \\
\hline 7 & 63.2 & 3 & 3 & 33.75 & 449 & 1518 & 1452 & 0.742 & 0.258 & 1742 & 0.522 & 0.478 & 0.123 & 3644 \\
\hline 7 & 63.2 & 3 & 4 & 35.35 & 381 & 1518 & 1452 & 0.687 & 0.313 & 1216 & 0.542 & 0.458 & 0.143 & 2656 \\
\hline \multicolumn{15}{|l|}{ Site 64} \\
\hline 7 & 64 & 1 & 2 & 2.75 & 649 & 1503 & 1448 & 0.723 & 0.217 & 2339 & 0.813 & 0.187 & 0.052 & 12509 \\
\hline 7 & 64 & 1 & 3 & 3.75 & 552 & 1503 & 1448 & 0.719 & 0.281 & 1962 & 0.806 & 0.194 & 0.055 & 10114 \\
\hline 7 & 64 & 1 & 4 & 5.25 & 395 & 1503 & 1448 & 0.713 & 0.287 & 1378 & 0.778 & 0.222 & 0.064 & 6205 \\
\hline 7 & 64 & 1 & 5 & 6.75 & 383 & 1503 & 1448 & 0.715 & 0.285 & 1342 & 0.826 & 0.174 & 0.050 & 7710 \\
\hline 7 & 64 & 1 & 6 & 8.25 & 380 & 1503 & 1448 & 0.719 & 0.281 & 1352 & 0.821 & 0.179 & 0.050 & 7553 \\
\hline 7 & 64 & 2 & 1 & 99.75 & 263 & 1504 & 1465 & 0.655 & 0.345 & 763 & 0.866 & 0.134 & 0.046 & 5696 \\
\hline 7 & 64 & 2 & 2 & 101.25 & 224 & 1504 & 1465 & 0.660 & 0.340 & 660 & 0.885 & 0.115 & 0.039 & 5741 \\
\hline 7 & 64 & 2 & 3 & 102.75 & 206 & 1504 & 1465 & 0.662 & 0.338 & 610 & 0.886 & 0.114 & 0.039 & 5353 \\
\hline 7 & 64 & 2 & 6 & 107.25 & 228 & 1504 & 1465 & 0.619 & 0.381 & 598 & 0.910 & 0.090 & 0.034 & 6642 \\
\hline 7 & 64 & 3. & 1 & 202.75 & 173 & 1501 & 1422 & 0.606 & 0.394 & 438 & 0.927 & 0.073 & 0.029 & 6001 \\
\hline 7 & 64 & 3 & 2 & 204.25 & 139 & 1501 & 1422 & 0.590 & 0.410 & 340 & 0.955 & 0.045 & 0.018 & 7563 \\
\hline 7 & 64 & 3 & 3 & 205.75 & 170 & 1501 & 1422 & 0.588 & 0.412 & 413 & 0.861 & 0.139 & 0.057 & 2969 \\
\hline 7 & 64 & 3 & 4 & 207.25 & 194 & 1501 & 1422 & 0.608 & 0.392 & 494 & 0.931 & 0.069 & 0.027 & 7159 \\
\hline 7 & 64 & 3 & 5 & 208.75 & 188 & 1501 & 1422 & 0.595 & 0.405 & 464 & 0.916 & 0.084 & 0.034 & 5522 \\
\hline 7 & 64 & 3 & 6 & 210.25 & 172 & 1501 & 1422 & 0.597 & 0.403 & 426 & 0.876 & 0.124 & 0.050 & 3438 \\
\hline 7 & 64 & 4 & 1 & 304.75 & 157 & 1489 & 1435 & 0.621 & 0.379 & 413 & 0.890 & 0.110 & 0.042 & 3757 \\
\hline 7 & 64 & 4 & 2 & 306.25 & 142 & 1489 & 1435 & 0.615 & 0.385 & 369 & 0.883 & 0.117 & 0.045 & 3151 \\
\hline 7 & 64 & 4 & 3 & 307.75 & 165 & 1489 & 1435 & 0.590 & 0.410 & 402 & 0.846 & 0.154 & 0.063 & 2607 \\
\hline 7 & 64 & 4 & 4 & 309.25 & 139 & 1489 & 1435 & 0.594 & 0.406 & 343 & 0.710 & 0.290 & 0.118 & 1132 \\
\hline 7 & 64 & 4 & 5 & 310.75 & 139 & 1489 & 1435 & 0.592 & 0.408 & 341 & 0.870 & 0.130 & 0.053 & 2622 \\
\hline
\end{tabular}


TABLE 1 - Continued

\begin{tabular}{|c|c|c|c|c|c|c|c|c|c|c|c|c|c|c|}
\hline Leg & Hole & Core & Section & $\begin{array}{l}\text { Depth Below } \\
\text { Sea Floor } \\
\text { (Meters) }\end{array}$ & $\begin{array}{c}\gamma^{\mathrm{a}} \\
\text { Gamma } \\
\text { Radiation }\end{array}$ & $\begin{array}{c}\text { Mean } \\
\text { Water } \\
\text { Control } \\
\end{array}$ & $\begin{array}{c}\text { Mean } \\
\text { Air } \\
\text { Control }\end{array}$ & $\begin{array}{c}\phi \\
\text { Porosity } \\
\text { Fraction } \\
\end{array}$ & $\begin{array}{c}1-\phi \\
\text { Solid } \\
\text { Fraction }\end{array}$ & $\gamma /(1-\phi)$ & $\begin{array}{c}\mathrm{CO}_{3} \\
\text { Weight } \\
\text { Fraction } \\
\end{array}$ & $\begin{array}{c}1-\mathrm{CO}_{3} \\
\text { Weight } \\
\text { Fraction }\end{array}$ & $\begin{array}{c}(1-\phi)\left(1-\mathrm{CO}_{3}\right) \\
\text { Weight } \\
\text { Fraction }\end{array}$ & $\gamma /(1-\phi)\left(1-\mathrm{CO}_{3}\right)$ \\
\hline \multicolumn{15}{|c|}{ Site 64 - Continued } \\
\hline 7 & 64 & 4 & 6 & 312.25 & 177 & 1489 & 1435 & 0.585 & 0.415 & 427 & 0.910 & 0.090 & 0.037 & 4739 \\
\hline 7 & 64 & 5 & 1 & 409.75 & 111 & 1489 & 1445 & 0.572 & 0.428 & 260 & 0.905 & 0.095 & 0.041 & 2737 \\
\hline 7 & 64 & 5 & 2 & 411.25 & 150 & 1489 & 1445 & 0.591 & 0.409 & 367 & 0.863 & 0.137 & 0.056 & 2632 \\
\hline 7 & 64 & 5 & 4 & 414.25 & 148 & 1489 & 1445 & 0.571 & 0.429 & 344 & 0.883 & 0.117 & 0.050 & 2944 \\
\hline 7 & 64 & 5 & 5 & 415.75 & 169 & 1489 & 1445 & 0.554 & 0.446 & 378 & 0.836 & 0.164 & 0.073 & 2305 \\
\hline 7 & 64 & 6 & 1 & 505.75 & 122 & 1467 & 1431 & 0.544 & 0.456 & 267 & 0.456 & 0.544 & 0.248 & 491 \\
\hline 7 & 64 & 7 & 1 & 610.75 & 110 & 1438 & 1430 & 0.605 & 0.395 & 278 & 0.847 & 0.153 & 0.060 & 1818 \\
\hline 7 & 64 & 7 & 3 & 613.75 & 130 & 1438 & 1430 & 0.562 & 0.438 & 297 & 0.869 & 0.131 & 0.057 & 2265 \\
\hline 7 & 64 & 7 & 4 & 615.25 & 118 & 1438 & 1430 & 0.548 & 0.452 & 261 & 0.886 & 0.114 & 0.051 & 2290 \\
\hline 7 & 64 & 7 & 6 & 618.25 & 104 & 1438 & 1430 & 0.586 & 0.414 & 250 & 0.910 & 0.090 & 0.037 & 2780 \\
\hline 7 & 64 & 7 & 5 & 616.75 & 132 & 1438 & 1430 & 0.575 & 0.425 & 310 & 0.894 & 0.106 & 0.045 & 2923 \\
\hline 7 & 64 & 8 & 2 & 707.25 & 126 & 1469 & 1422 & 0.471 & 0.529 & 237 & 0.859 & 0.141 & 0.075 & 1682 \\
\hline 7 & 64 & 8 & 3 & 708.75 & 152 & 1469 & 1422 & 0.469 & 0.531 & 287 & 0.861 & 0.139 & 0.074 & 2064 \\
\hline 7 & 64 & 10 & 1 & 848.75 & 192 & 1504 & 1410 & 0.500 & 0.500 & 385 & 0.879 & 0.121 & 0.060 & 3183 \\
\hline 7 & 64 & 10 & 2 & 850.25 & 149 & 1504 & 1410 & 0.452 & 0.548 & 272 & 0.845 & 0.155 & 0.085 & 1752 \\
\hline 7 & 64.1 & 1 & 1 & 433.75 & 116 & 1512 & 1445 & 0.546 & 0.454 & 255 & 0.907 & 0.093 & 0.042 & 2737 \\
\hline 7 & 64.1 & 1 & 2 & 435.25 & 149 & 1512 & 1445 & 0.550 & 0.450 & 332 & 0.797 & 0.203 & 0.091 & 1634 \\
\hline 7 & 64.1 & 1 & 3 & 436.75 & 147 & 1512 & 1445 & 0.559 & 0.441 & 333 & 0.693 & 0.307 & 0.135 & 1085 \\
\hline 7 & 64.1 & 1 & 4 & 438.25 & 157 & 1512 & 1445 & 0.551 & 0.449 & 351 & 0.901 & 0.099 & 0.044 & 3541 \\
\hline 7 & 64.1 & 1 & 5 & 439.75 & 128 & 1512 & 1445 & 0.532 & 0.468 & 273 & 0.908 & 0.092 & 0.043 & 2970 \\
\hline 7 & 64.1 & 1 & 6 & 441.25 & 157 & 1512 & 1445 & 0.566 & 0.434 & 362 & 0.854 & 0.136 & 0.059 & 2663 \\
\hline 7 & 64.1 & 2 & 1 & 442.75 & 163 & 1522 & 1459 & 0.587 & 0.413 & 395 & 0.836 & 0.164 & 0.068 & 2408 \\
\hline 7 & 64.1 & 2 & 2 & 444.25 & 159 & 1522 & 1459 & 0.574 & 0.426 & 372 & 0.850 & 0.150 & 0.064 & 2482 \\
\hline 7 & 64.1 & 2 & 3 & 445.75 & 140 & 1522 & 1459 & 0.547 & 0.453 & 309 & 0.820 & 0.180 & 0.081 & 1720 \\
\hline 7 & 64.1 & 2 & 4 & 447.25 & 168 & 1522 & 1459 & 0.531 & 0.469 & 357 & 0.886 & 0.114 & 0.054 & 3133 \\
\hline
\end{tabular}


TABLE 1 - Continued

\begin{tabular}{|c|c|c|c|c|c|c|c|c|c|c|c|c|c|c|}
\hline Leg & Hole & Core & Section & $\begin{array}{l}\text { Depth Below } \\
\text { Sea Floor } \\
\text { (Meters) }\end{array}$ & $\begin{array}{c}\gamma^{\mathrm{a}} \\
\text { Gamma } \\
\text { Radiation }\end{array}$ & $\begin{array}{c}\text { Mean } \\
\text { Water } \\
\text { Control }\end{array}$ & $\begin{array}{l}\text { Mean } \\
\text { Air } \\
\text { Control }\end{array}$ & $\begin{array}{c}\phi \\
\text { Porosity } \\
\text { Fraction }\end{array}$ & $\begin{array}{c}1-\phi \\
\text { Solid } \\
\text { Fraction }\end{array}$ & $\gamma /(1-\phi)$ & $\begin{array}{c}\mathrm{CO}_{3} \\
\text { Weight } \\
\text { Fraction }\end{array}$ & $\begin{array}{c}1-\mathrm{CO}_{3} \\
\text { Weight } \\
\text { Fraction }\end{array}$ & $\begin{array}{c}(1-\phi)\left(1-\mathrm{CO}_{3}\right) \\
\text { Weight } \\
\text { Fraction }\end{array}$ & $\gamma /(1-\phi)\left(1-\mathrm{CO}_{3}\right)$ \\
\hline \multicolumn{15}{|c|}{ Site $64-$ Continued } \\
\hline 7 & 64.1 & 2 & 5 & 448.75 & 134 & 1522 & 1459 & 0.544 & 0.456 & 294 & 0.886 & 0.114 & 0.052 & 2577 \\
\hline 7 & 64.1 & 2 & 6 & 450.25 & 151 & 1522 & 1459 & 0.532 & 0.468 & 323 & 0.915 & 0.085 & 0.040 & 3802 \\
\hline 7 & 64.1 & 3 & 1 & 451.75 & 159 & 1475 & 1435 & 0.531 & 0.469 & 339 & 0.893 & 0.107 & 0.050 & 3171 \\
\hline 7 & 64.1 & 3 & 2 & 453.25 & 153 & 1475 & 1435 & 0.535 & 0.445 & 343 & 0.874 & 0.126 & 0.056 & 2723 \\
\hline 7 & 64.1 & 3 & 3 & 454.75 & 121 & 1475 & 1435 & 0.542 & 0.458 & 265 & 0.901 & 0.099 & 0.045 & 2674 \\
\hline 7 & 64.1 & 3 & 4 & 456.25 & 121 & 1475 & 1435 & 0.536 & 0.464 & 261 & 0.896 & 0.104 & 0.048 & 2509 \\
\hline 7 & 64.1 & 3 & 5 & 457.75 & 142 & 1475 & 1435 & 0.556 & 0.444 & 320 & 0.929 & 0.071 & 0.032 & 4504 \\
\hline 7 & 64.1 & 3 & 6 & 459.25 & 144 & 1475 & 1435 & 0.555 & 0.445 & 323 & 0.908 & 0.092 & 0.041 & 3513 \\
\hline 7 & 64.1 & 4 & 1 & 461.75 & 157 & 1475 & 1450 & 0.568 & 0.432 & 363 & 0.866 & 0.134 & 0.058 & 2711 \\
\hline 7 & 64.1 & 4 & 2 & 463.25 & 132 & 1475 & 1450 & 0.562 & 0.438 & 302 & 0.903 & 0.097 & 0.042 & 3111 \\
\hline 7 & 64.1 & 4 & 4 & 466.25 & 161 & 1475 & 1450 & 0.566 & 0.434 & 370 & 0.865 & 0.135 & 0.059 & 2741 \\
\hline 7 & 64.1 & 4 & 5 & 467.75 & 153 & 1475 & 1450 & 0.540 & 0.460 & 334 & 0.840 & 0.160 & 0.074 & 2085 \\
\hline 7 & 64.1 & 4 & 6 & 469.25 & 157 & 1475 & 1450 & 0.528 & 0.472 & 333 & 0.875 & 0.125 & 0.059 & 2663 \\
\hline 7 & 64.1 & 5 & 1 & 470.75 & 134 & 1494 & 1483 & 0.579 & 0.421 & 319 & 0.828 & 0.172 & 0.072 & 1856 \\
\hline 7 & 64.1 & 5 & 2 & 472.25 & 163 & 1494 & 1483 & 0.556 & 0.444 & 366 & 0.850 & 0.150 & 0.067 & 2442 \\
\hline 7 & 64.1 & 5 & 3 & 473.75 & 149 & 1494 & 1483 & 0.540 & 0.460 & 324 & 0.858 & 0.142 & 0.065 & 2233 \\
\hline 7 & 64.1 & 5 & 4 & 475.25 & 153 & 1494 & 1483 & 0.523 & 0.477 & 320 & 0.902 & 0.098 & 0.047 & 3263 \\
\hline 7 & 64.1 & 5 & 5 & 476.75 & 143 & 1494 & 1483 & 0.509 & 0.491 & 291 & 0.923 & 0.077 & 0.038 & 3779 \\
\hline 7 & 64.1 & 5 & 6 & 478.25 & 144 & 1494 & 1483 & 0.523 & 0.476 & 303 & 0.886 & 0.114 & 0.054 & 2656 \\
\hline 7 & 64.1 & 6 & 1 & 565.75 & 158 & 1502 & 1436 & 0.512 & 0.488 & 323 & 0.886 & 0.114 & 0.056 & 2837 \\
\hline 7 & 64.1 & 6 & 2 & 567.25 & 137 & 1502 & 1436 & 0.529 & 0.471 & 290 & 0.875 & 0.125 & 0.059 & 2321 \\
\hline 7 & 64.1 & 6 & 3 & 568.75 & 152 & 1502 & 1436 & 0.526 & 0.474 & 320 & 0.871 & 0.129 & 0.061 & 2432 \\
\hline 7 & 64.1 & 6 & 4 & 570.25 & 110 & 1502 & 1436 & 0.521 & 0.479 & 229 & 0.916 & 0.084 & 0.040 & 2723 \\
\hline 7 & 64.1 & 7 & 1 & 661.75 & 148 & 1507 & 1480 & 0.557 & 0.443 & 335 & 0.858 & 0.142 & 0.063 & 2356 \\
\hline 7 & 64.1 & 7 & 2 & 663.25 & 154 & 1507 & 1480 & 0.516 & 0.484 & 318 & 0.810 & 0.190 & 0.092 & 1672 \\
\hline
\end{tabular}


TABLE 1 - Continued

\begin{tabular}{|c|c|c|c|c|c|c|c|c|c|c|c|c|c|c|}
\hline Leg & Hole & Core & Section & $\begin{array}{l}\text { Depth Below } \\
\text { Sea Floor } \\
\text { (Meters) }\end{array}$ & $\begin{array}{c}\gamma^{\mathrm{a}} \\
\text { Gamma } \\
\text { Radiation }\end{array}$ & \begin{tabular}{|c} 
Mean \\
Water \\
Control
\end{tabular} & $\begin{array}{c}\text { Mean } \\
\text { Air } \\
\text { Control }\end{array}$ & $\begin{array}{c}\phi \\
\text { Porosity } \\
\text { Fraction }\end{array}$ & $\begin{array}{c}1-\phi \\
\text { Solid } \\
\text { Fraction } \\
\end{array}$ & $\gamma /(1-\phi)$ & $\begin{array}{c}\mathrm{CO}_{3} \\
\text { Weight } \\
\text { Fraction }\end{array}$ & $\begin{array}{l}1-\mathrm{CO}_{3} \\
\text { Weight } \\
\text { Fraction }\end{array}$ & $\begin{array}{c}(1-\phi)\left(1-\mathrm{CO}_{3}\right) \\
\text { Weight } \\
\text { Fraction } \\
\end{array}$ & $\gamma /(1-\phi)\left(1-\mathrm{CO}_{3}\right)$ \\
\hline \multicolumn{15}{|c|}{ Site $64-$ Continued } \\
\hline 7 & 64.1 & 7 & 3 & 664.75 & 161 & 1507 & 1480 & 0.515 & 0.485 & 333 & 0.843 & 0.157 & 0.076 & 2120 \\
\hline 7 & 64.1 & 7 & 4 & 666.25 & 147 & 1507 & 1480 & 0.536 & 0.464 & 316 & 0.908 & 0.092 & 0.043 & 3433 \\
\hline 7 & 64.1 & 8 & 2 & 748.25 & 168 & 1455 & 1439 & 0.487 & 0.513 & 326 & 0.893 & 0.107 & 0.055 & 3050 \\
\hline 7 & 64.1 & 9 & 2 & 913.25 & 113 & 1500 & 1477 & 0.515 & 0.485 & 234 & 0.920 & 0.080 & 0.039 & 2921 \\
\hline 7 & 64.1 & 9 & 3 & 914.75 & 90 & 1500 & 1477 & 0.490 & 0.510 & 176 & 0.921 & 0.079 & 0.040 & 2231 \\
\hline 7 & 64.1 & 10 & 1 & 969.75 & 109 & 1443 & 1437 & 0.517 & 0.483 & 227 & 0.826 & 0.174 & 0.084 & 1302 \\
\hline 7 & 64.1 & 10 & 2 & 971.25 & 145 & 1443 & 1437 & 0.494 & 0.506 & 286 & 0.840 & 0.160 & 0.081 & 1788 \\
\hline \multicolumn{15}{|l|}{ Site 65 } \\
\hline 7 & 65 & 2 & 4 & 15.25 & 333 & 1488 & 1440 & 0.916 & 0.084 & 3947 & 0.004 & 0.996 & 0.084 & 3963 \\
\hline 7 & 65 & 2 & 5 & 16.75 & 302 & 1488 & 1440 & 0.911 & 0.089 & 3396 & 0.006 & 0.994 & 0.088 & 3416 \\
\hline 7 & 65 & 3 & 1 & 19.75 & 326 & 1509 & 1435 & 0.907 & 0.093 & 3524 & 0.004 & 0.996 & 0.092 & 3538 \\
\hline 7 & 65 & 3 & 2 & 21.25 & 312 & 1509 & 1435 & 0.912 & 0.088 & 3538 & 0.007 & 0.993 & 0.088 & 3563 \\
\hline 7 & 65 & 3 & 3 & 22.75 & 369 & 1509 & 1435 & 0.912 & 0.088 & 4176 & 0.007 & 0.993 & 0.088 & 4205 \\
\hline 7 & 65 & 3 & 4 & 24.25 & 343 & 1509 & 1435 & 0.900 & 0.100 & 3419 & 0.005 & 0.995 & 0.100 & 3436 \\
\hline 7 & 65 & 3 & 5 & 25.75 & 369 & 1509 & 1435 & 0.901 & 0.099 & 3725 & 0.007 & 0.993 & 0.098 & 3751 \\
\hline 7 & 65 & 5 & 2 & 39.25 & 342 & 1482 & 1454 & 0.898 & 0.102 & 3354 & 0.004 & 0.996 & 0.102 & 3368 \\
\hline 7 & 65 & 5 & 4 & 42.25 & 299 & 1482 & 1454 & 0.894 & 0.106 & 2833 & 0.002 & 0.998 & 0.105 & 2839 \\
\hline 7 & 65 & 5 & 5 & 43.75 & 309 & 1482 & 1454 & 0.908 & 0.092 & 3344 & 0.016 & 0.984 & 0.091 & 3398 \\
\hline 7 & 65 & 5 & 6 & 45.25 & 283 & 1482 & 1454 & 0.922 & 0.078 & 3624 & 0.011 & 0.989 & 0.077 & 3665 \\
\hline
\end{tabular}


TABLE 1 - Continued

\begin{tabular}{|c|c|c|c|c|c|c|c|c|c|c|c|c|c|c|}
\hline Leg & Hole & Core & Section & $\begin{array}{l}\text { Depth Below } \\
\text { Sea Floor } \\
\text { (Meters) }\end{array}$ & $\begin{array}{c}\gamma^{\mathrm{a}} \\
\text { Gamma } \\
\text { Radiation }\end{array}$ & $\begin{array}{l}\text { Mean } \\
\text { Water } \\
\text { Control }\end{array}$ & \begin{tabular}{|c|} 
Mean \\
Air \\
Control
\end{tabular} & $\begin{array}{c}\phi \\
\text { Porosity } \\
\text { Fraction }\end{array}$ & $\begin{array}{c}1-\phi \\
\text { Solid } \\
\text { Fraction }\end{array}$ & $\gamma /(1-\phi)$ & $\begin{array}{c}\mathrm{CO}_{3} \\
\text { Weight } \\
\text { Fraction }\end{array}$ & $\begin{array}{c}1-\mathrm{CO}_{3} \\
\text { Weight } \\
\text { Fraction }\end{array}$ & $\begin{array}{c}(1-\phi)\left(1-\mathrm{CO}_{3}\right) \\
\text { Weight } \\
\text { Fraction }\end{array}$ & $\gamma /(1-\phi)\left(1-\mathrm{CO}_{3}\right)$ \\
\hline \multicolumn{15}{|c|}{ Site 65-Continued } \\
\hline 7 & 65 & 7 & 2 & 57.25 & 348 & 1456 & 1411 & 0.891 & 0.109 & 3189 & 0.006 & 0.994 & 0.108 & 3208 \\
\hline 7 & 65 & 7 & 3 & 58.75 & 353 & 1456 & 1411 & 0.884 & 0.116 & 3028 & 0.010 & 0.990 & 0.115 & 3058 \\
\hline 7 & 65 & 7 & 4 & 60.25 & 370 & 1456 & 1411 & 0.903 & 0.097 & 3804 & 0.005 & 0.995 & 0.097 & 3823 \\
\hline 7 & 65 & 7 & 5 & 61.75 & 350 & 1456 & 1411 & 0.911 & 0.089 & 3927 & 0.004 & 0.996 & 0.089 & 3942 \\
\hline 7 & 65 & 8 & 1 & 64.75 & 324 & 1446 & 1445 & 0.872 & 0.128 & 2526 & 0.008 & 0.992 & 0.127 & 2546 \\
\hline 7 & 65 & 8 & 2 & 66.25 & 331 & 1446 & 1445 & 0.869 & 0.131 & 2528 & 0.004 & 0.996 & 0.130 & 2538 \\
\hline 7 & 65 & 8 & 3 & 67.75 & 302 & 1446 & 1445 & 0.847 & 0.153 & 1970 & 0.030 & 0.970 & 0.149 & 2031 \\
\hline 7 & 65 & 8 & 4 & 69.25 & 322 & 1446 & 1445 & 0.884 & 0.116 & 2783 & 0.006 & 0.994 & 0.115 & 2800 \\
\hline 7 & 65 & 8 & 5 & 70.75 & 305 & 1446 & 1445 & 0.855 & 0.145 & 2107 & 0.006 & 0.994 & 0.144 & 2120 \\
\hline 7 & 65 & 8 & 6 & 72.25 & 332 & 1446 & 1445 & 0.864 & 0.136 & 2437 & 0.049 & 0.951 & 0.130 & 2562 \\
\hline 7 & 65 & 9 & 1 & 74.75 & 315 & 1481 & 1490 & 0.843 & 0.157 & 2015 & 0.003 & 0.997 & 0.156 & 2021 \\
\hline 7 & 65 & 9 & 2 & 76.25 & 310 & 1481 & 1490 & 0.850 & 0.150 & 2072 & 0.015 & 0.985 & 0.147 & 2103 \\
\hline 7 & 65 & 9 & 3 & 77.75 & 246 & 1481 & 1490 & 0.845 & 0.155 & 1589 & 0.006 & 0.994 & 0.154 & 1599 \\
\hline 7 & 65 & 9 & 5 & 80.75 & 283 & 1481 & 1490 & 0.353 & 0.147 & 1925 & 0.003 & 0.997 & 0.147 & 1930 \\
\hline 7 & 65 & 10 & 1 & 83.75 & 213 & 1480 & 1443 & 0.359 & 0.141 & 1508 & 0.012 & 0.988 & 0.139 & 1526 \\
\hline 7 & 65 & 10 & 3 & 86.75 & 271 & 1480 & 1443 & 0.845 & 0.155 & 1750 & 0.018 & 0.982 & 0.152 & 1782 \\
\hline 7 & 65 & 10 & 4 & 88.25 & 263 & 1480 & 1443 & 0.847 & 0.153 & 1715 & 0 & 1.000 & 0.153 & 1715 \\
\hline 7 & 65 & 11 & 2 & 94.25 & 254 & 1501 & 1433 & 0.890 & 0.110 & 2299 & 0.010 & 0.990 & 0.109 & 2323 \\
\hline 7 & 65 & 11 & 3 & 95.75 & 266 & 1501 & 1433 & 0.887 & 0.113 & 2348 & 0.003 & 0.997 & 0.113 & 2355 \\
\hline 7 & 65 & 11 & 4 & 97.25 & 274 & 1501 & 1433 & 0.897 & 0.103 & 2652 & 0.004 & 0.996 & 0.103 & 2663 \\
\hline 7 & 65 & 11 & 5 & 98.75 & 280 & 1501 & 1433 & 0.913 & 0.087 & 3235 & 0.007 & 0.993 & 0.086 & 3258 \\
\hline 7 & 65 & 11 & 6 & 100.25 & 266 & 1501 & 1433 & 0.923 & 0.077 & 3478 & 0 & 1.000 & 0.077 & 3478 \\
\hline 7 & 65 & 12 & 1 & 101.75 & 293 & 1483 & 1460 & 0.937 & 0.063 & 4673 & 0.015 & 0.985 & 0.062 & 4744 \\
\hline 7 & 65 & 12 & 2 & 103.25 & 318 & 1483 & 1460 & 0.892 & 0.108 & 2945 & 0.003 & 0.997 & 0.108 & 2954 \\
\hline 7 & 65 & 12 & 3 & 104.75 & 293 & 1483 & 1460 & 0.890 & 0.110 & 2658 & 0.003 & 0.997 & 0.110 & 2666 \\
\hline
\end{tabular}


TABLE 1 - Continued

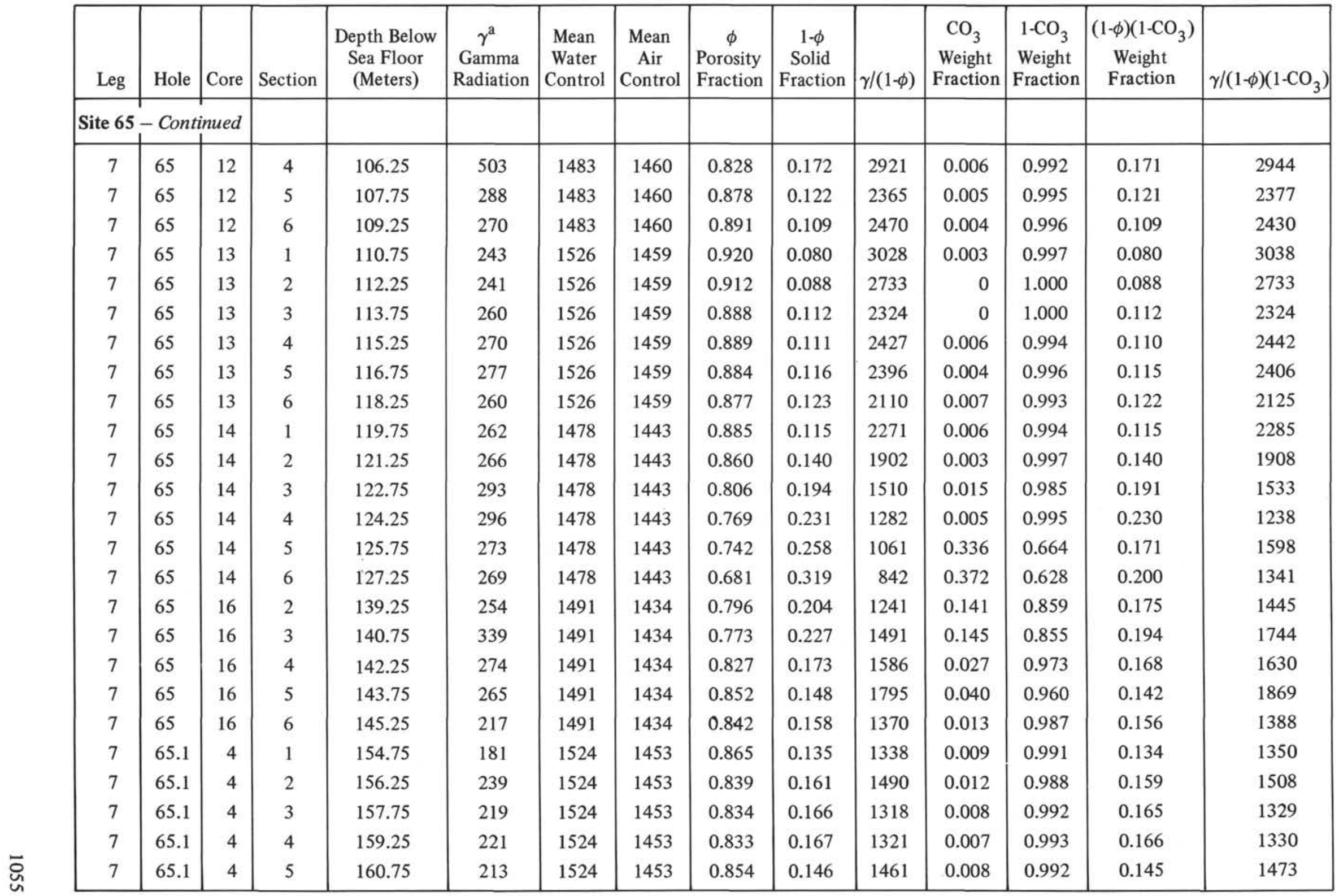


TABLE 1 - Continued

\begin{tabular}{|c|c|c|c|c|c|c|c|c|c|c|c|c|c|c|}
\hline Leg & Hole & Core & Section & $\begin{array}{l}\text { Depth Below } \\
\text { Sea Floor } \\
\text { (Meters) }\end{array}$ & $\begin{array}{c}\gamma^{\mathrm{a}} \\
\text { Gamma } \\
\text { Radiation }\end{array}$ & \begin{tabular}{|c} 
Mean \\
Water \\
Control
\end{tabular} & \begin{tabular}{|c} 
Mean \\
Air \\
Control \\
\end{tabular} & $\begin{array}{c}\phi \\
\text { Porosity } \\
\text { Fraction }\end{array}$ & $\begin{array}{c}1-\phi \\
\text { Solid } \\
\text { Fraction }\end{array}$ & $\gamma /(1-\phi)$ & $\begin{array}{c}\mathrm{CO}_{3} \\
\text { Weight } \\
\text { Fraction } \\
\end{array}$ & $\begin{array}{c}1-\mathrm{CO}_{3} \\
\text { Weight } \\
\text { Fraction }\end{array}$ & $\begin{array}{c}(1-\phi)\left(1-\mathrm{CO}_{3}\right) \\
\text { Weight } \\
\text { Fraction }\end{array}$ & $\gamma /(1-\phi)\left(1-\mathrm{CO}_{3}\right)$ \\
\hline \multicolumn{15}{|c|}{ Site 65 - Continued } \\
\hline 7 & 65.1 & 4 & 6 & 162.25 & 194 & 1524 & 1453 & 0.862 & 0.138 & 1409 & 0.010 & 0.990 & 0.136 & 1424 \\
\hline 7 & 65.1 & 5 & 1 & 162.75 & 259 & 1518 & 1432 & 0.799 & 0.201 & 1290 & 0.019 & 0.981 & 0.197 & 1315 \\
\hline 7 & 65.1 & 5 & 2 & 164.25 & 211 & 1518 & 1432 & 0.846 & 0.154 & 1370 & 0.009 & 0.991 & 0.152 & 1383 \\
\hline 7 & 65.1 & 5 & 3 & 165.75 & 222 & 1518 & 1432 & 0.832 & 0.168 & 1323 & 0.010 & 0.990 & 0.166 & 1337 \\
\hline 7 & 65.1 & 5 & 4 & 167.25 & 263 & 1518 & 1432 & 0.820 & 0.179 & 1472 & 0.007 & 0.993 & 0.178 & 1432 \\
\hline \multicolumn{15}{|l|}{ Site 66} \\
\hline 7 & 66 & 2 & 1 & 79.75 & 245 & 1489 & 1458 & 0.920 & 0.080 & 3076 & 0 & 1.000 & 0.080 & 3076 \\
\hline 7 & 66 & 2 & 2 & 81.25 & 220 & 1489 & 1458 & 0.927 & 0.073 & 3014 & 0 & 1.000 & 0.073 & 3014 \\
\hline 7 & 66 & 2 & 3 & 82.75 & 210 & 1489 & 1458 & 0.938 & 0.062 & 3378 & 0 & 1.000 & 0.062 & 3378 \\
\hline 7 & 66 & 3 & 1 & 117.75 & 273 & 1512 & 1448 & 0.881 & 0.119 & 2296 & 0 & 1.000 & 0.119 & 2297 \\
\hline 7 & 66 & 3 & 2 & 119.25 & 257 & 1512 & 1448 & 0.858 & 0.142 & 1814 & 0.012 & 0.988 & 0.140 & 1836 \\
\hline 7 & 66 & 3 & 3 & 120.75 & 312 & 1512 & 1448 & 0.870 & 0.130 & 2402 & 0 & 1.000 & 0.130 & 2402 \\
\hline 7 & 66 & 3 & 6 & 125.25 & 332 & 1512 & 1448 & 0.879 & 0.121 & 2733 & 0 & 1.000 & 0.121 & 2733 \\
\hline 7 & 66 & 6 & 1 & 165.75 & 1669 & 1511 & 1453 & 0.625 & 0.375 & 4449 & 0.002 & 0.998 & 0.374 & 4458 \\
\hline 7 & 66 & 6 & 2 & 167.25 & 1782 & 1511 & 1453 & 0.708 & 0.292 & 6111 & 0.001 & 0.999 & 0.291 & 6117 \\
\hline 7 & 66 & 6 & 3 & 168.75 & 1917 & 1511 & 1453 & 0.700 & 0.300 & 6397 & 0.002 & 0.998 & 0.299 & 6410 \\
\hline 7 & 66 & 6 & 4 & 170.25 & 2002 & 1511 & 1453 & 0.679 & 0.321 & 6235 & 0 & 1.000 & 0.321 & 6235 \\
\hline 7 & 66 & 7 & 1 & 174.75 & 1867 & 1509 & 1421 & 0.691 & 0.309 & 6033 & 0.002 & 0.998 & 0.309 & 6045 \\
\hline 7 & 66 & 7 & 2 & 176.25 & 2202 & 1509 & 1421 & 0.658 & 0.342 & 6441 & 0.001 & 0.999 & 0.341 & 6448 \\
\hline 7 & 66 & 7 & 3 & 177.75 & 2360 & 1509 & 1421 & 0.659 & 0.341 & 6926 & 0 & 1.000 & 0.341 & 6926 \\
\hline 7 & 66 & 7 & 4 & 179.25 & 2186 & 1509 & 1421 & 0.649 & 0.351 & 6230 & 0.001 & 0.999 & 0.350 & 6236 \\
\hline 7 & 66 & 8 & 1 & 180.75 & 2052 & 1538 & 1474 & 0.709 & 0.291 & 7061 & 0 & 1.000 & 0.291 & 7061 \\
\hline 7 & 66 & 8 & 2 & 182.25 & 1831 & 1538 & 1474 & 0.728 & 0.274 & 6679 & 0 & 1.000 & 0.274 & 6679 \\
\hline 7 & 66 & 8 & 3 & 183.75 & 1767 & 1506 & 1428 & 0.774 & 0.226 & 7806 & 0 & 1.000 & 0.226 & 7806 \\
\hline
\end{tabular}


TABLE 1 - Continued

\begin{tabular}{|c|c|c|c|c|c|c|c|c|c|c|c|c|c|c|}
\hline Leg & Hole & Core & Section & $\begin{array}{c}\text { Depth Below } \\
\text { Sea Floor } \\
\text { (Meters) }\end{array}$ & $\begin{array}{c}\gamma^{\mathrm{a}} \\
\text { Gamma } \\
\text { Radiation }\end{array}$ & \begin{tabular}{|c|} 
Mean \\
Water \\
Control
\end{tabular} & $\begin{array}{c}\text { Mean } \\
\text { Air } \\
\text { Control }\end{array}$ & $\begin{array}{c}\phi \\
\text { Porosity } \\
\text { Fraction }\end{array}$ & $\begin{array}{c}1-\phi \\
\text { Solid } \\
\text { Fraction }\end{array}$ & $\gamma /(1-\phi)$ & $\begin{array}{c}\mathrm{CO}_{3} \\
\text { Weight } \\
\text { Fraction } \\
\end{array}$ & $\begin{array}{l}1-\mathrm{CO}_{3} \\
\text { Weight } \\
\text { Fraction } \\
\end{array}$ & $\begin{array}{c}(1-\phi)\left(1-\mathrm{CO}_{3}\right) \\
\text { Weight } \\
\text { Fraction }\end{array}$ & $\gamma /(1-\phi)\left(1-\mathrm{CO}_{3}\right)$ \\
\hline \multicolumn{15}{|c|}{ Site 66 -Continued } \\
\hline 7 & 66 & 8 & 4 & 185.25 & 1597 & 1506 & 1428 & 0.799 & 0.201 & 7930 & 0 & 1.000 & 0.201 & 7903 \\
\hline 7 & 66 & 8 & 5 & 186.75 & 1633 & 1506 & 1428 & 0.798 & 0.204 & 8016 & 0 & 1.000 & 0.204 & 8016 \\
\hline 7 & 66 & 9 & 1 & 187.75 & 855 & 1496 & 1448 & 0.762 & 0.238 & 3592 & 0 & 1.000 & 0.238 & 3592 \\
\hline 7 & 66 & 9 & 3 & 190.75 & 948 & 1496 & 1448 & 0.777 & 0.223 & 4250 & 0.002 & 0.998 & 0.223 & 4258 \\
\hline 7 & 66.1 & 2 & 2 & 22.25 & 341 & 1483 & 1498 & 0.901 & 0.099 & 3463 & 0.006 & 0.994 & 0.098 & 3484 \\
\hline 7 & 66.1 & 2 & 3 & 23.75 & 349 & 1483 & 1498 & 0.909 & 0.091 & 3837 & 0 & 1.000 & 0.091 & 3837 \\
\hline 7 & 66.1 & 2 & 4 & 25.25 & 321 & 1483 & 1498 & 0.903 & 0.097 & 3318 & 0 & 1.000 & 0.097 & 3318 \\
\hline 7 & 66.1 & 2 & 5 & 26.75 & 241 & 1483 & 1498 & 0.905 & 0.095 & 2539 & 0.005 & 0.995 & 0.095 & 2551 \\
\hline 7 & 66.1 & 2 & 6 & 28.25 & 281 & 1483 & 1498 & 0.912 & 0.088 & 3192 & 0.009 & 0.991 & 0.087 & 3221 \\
\hline 7 & 66.1 & 3 & 2 & 31.25 & 268 & 1521 & 1438 & 0.875 & 0.125 & 2143 & 0.002 & 0.998 & 0.125 & 2147 \\
\hline 7 & 66.1 & 3 & 3 & 32.75 & 287 & 1521 & 1438 & 0.864 & 0.136 & 2109 & 0 & 1.000 & 0.136 & 2109 \\
\hline 7 & 66.1 & 3 & 4 & 34.25 & 296 & 1521 & 1438 & 0.850 & 0.150 & 1978 & 0 & 1.000 & 0.150 & 1978 \\
\hline 7 & 66.1 & 3 & 5 & 35.75 & 266 & 1521 & 1438 & 0.866 & 0.134 & 1985 & 0 & 1.000 & 0.134 & 1985 \\
\hline 7 & 66.1 & 3 & 6 & 37.25 & 251 & 1521 & 1438 & 0.865 & 0.135 & 1851 & 0 & 1.000 & 0.135 & 1851 \\
\hline 7 & 66.1 & 4 & 1 & 38.75 & 227 & 1512 & 1448 & 0.860 & 0.140 & 1620 & 0 & 1.000 & 0.140 & 1620 \\
\hline 7 & 66.1 & 4 & 2 & 40.25 & 248 & 1512 & 1448 & 0.881 & 0.119 & 2084 & 0 & 1.000 & 0.119 & 2084 \\
\hline 7 & 66.1 & 4 & 3 & 41.75 & 237 & 1512 & 1448 & 0.881 & 0.119 & 1989 & 0 & 1.000 & 0.119 & 1939 \\
\hline 7 & 66.1 & 4 & 4 & 43.25 & 278 & 1512 & 1448 & 0.893 & 0.107 & 2601 & 0 & 1.000 & 0.107 & 2601 \\
\hline 7 & 66.1 & 4 & 5 & 44.75 & 238 & 1512 & 1448 & 0.902 & 0.098 & 2424 & 0 & 1.000 & 0.098 & 2424 \\
\hline 7 & 66.1 & 4 & 6 & 46.25 & 150 & 1512 & 1448 & 0.893 & 0.107 & 1398 & 0 & 1.000 & 0.107 & 1398 \\
\hline 7 & 66.1 & 5 & 1 & 47.75 & 281 & 1526 & 1456 & 0.864 & 0.136 & 2068 & 0 & 1.000 & 0.136 & 2068 \\
\hline 7 & 66.1 & 5 & 2 & 49.25 & 284 & 1526 & 1456 & 0.874 & 0.126 & 2256 & 0 & 1.000 & 0.126 & 2256 \\
\hline 7 & 66.1 & 5 & 3 & 50.75 & 291 & 1526 & 1456 & 0.887 & 0.113 & 2568 & 0.012 & 0.988 & 0.112 & 2599 \\
\hline 7 & 66.1 & 5 & 4 & 52.25 & 284 & 1526 & 1456 & 0.872 & 0.128 & 2220 & 0 & 1.000 & 0.128 & 2220 \\
\hline 7 & 66.1 & 5 & 5 & 53.75 & 285 & 1526 & 1456 & 0.872 & 0.128 & 2228 & 0 & 1.000 & 0.128 & 2228 \\
\hline 7 & 66.1 & 5 & 6 & 55.25 & 248 & 1526 & 1456 & 0.883 & 0.117 & 2126 & 0 & 1.000 & 0.117 & 2126 \\
\hline
\end{tabular}


TABLE 1 - Continued

\begin{tabular}{|c|c|c|c|c|c|c|c|c|c|c|c|c|c|c|}
\hline Leg & Hole & Core & Section & $\begin{array}{l}\text { Depth Below } \\
\text { Sea Floor } \\
\text { (Meters) } \\
\end{array}$ & $\begin{array}{c}\gamma^{\mathrm{a}} \\
\text { Gamma } \\
\text { Radiation }\end{array}$ & \begin{tabular}{|c} 
Mean \\
Water \\
Control \\
\end{tabular} & \begin{tabular}{|c} 
Mean \\
Air \\
Control \\
\end{tabular} & $\begin{array}{c}\phi \\
\text { Porosity } \\
\text { Fraction } \\
\end{array}$ & $\begin{array}{c}1-\phi \\
\text { Solid } \\
\text { Fraction }\end{array}$ & $\gamma /(1-\phi)$ & $\begin{array}{c}\mathrm{CO}_{3} \\
\text { Weight } \\
\text { Fraction }\end{array}$ & $\begin{array}{c}1-\mathrm{CO}_{3} \\
\text { Weight } \\
\text { Fraction }\end{array}$ & $\begin{array}{c}(1-\phi)\left(1-\mathrm{CO}_{3}\right) \\
\text { Weight } \\
\text { Fraction } \\
\end{array}$ & $\gamma /(1-\phi)\left(1-\mathrm{CO}_{3}\right)$ \\
\hline \multicolumn{15}{|c|}{ Site 66 - Continued } \\
\hline 7 & 66.1 & 6 & 2 & 58.25 & 258 & 1510 & 1434 & 0.889 & 0.111 & 2324 & 0 & 1.000 & 0.111 & 2324 \\
\hline 7 & 66.1 & 6 & 3 & 59.75 & 293 & 1510 & 1434 & 0.878 & 0.122 & 2403 & 0 & 1.000 & 0.122 & 2403 \\
\hline 7 & 66.1 & 6 & 4 & 61.25 & 313 & 1510 & 1434 & 0.867 & 0.133 & 2360 & 0 & 1.000 & 0.133 & 2360 \\
\hline 7 & 66.1 & 6 & 5 & 62.75 & 294 & 1510 & 1434 & 0.873 & 0.127 & 2309 & 0 & 1.000 & 0.127 & 2309 \\
\hline 7 & 66.1 & 6 & 6 & 64.25 & 253 & 1510 & 1434 & 0.869 & 0.131 & 1934 & 0 & 1.000 & 0.131 & 1934 \\
\hline 7 & 66.1 & 7 & 1 & 67.75 & 312 & 1527 & 1424 & 0.903 & 0.107 & 3226 & 0 & 1.000 & 0.097 & 3226 \\
\hline 7 & 66.1 & 7 & 2 & 69.25 & 302 & 1527 & 1424 & 0.886 & 0.114 & 2646 & 0 & 1.000 & 0.114 & 2646 \\
\hline 7 & 66.1 & 7 & 3 & 70.75 & 279 & 1527 & 1424 & 0.859 & 0.141 & 1970 & 0.011 & 0.989 & 0.141 & 1992 \\
\hline 7 & 66.1 & 7 & 4 & 72.25 & 299 & 1527 & 1424 & 0.869 & 0.131 & 2290 & 0 & 1.000 & 0.131 & 2290 \\
\hline 7 & 66.1 & 7 & 5 & 73.75 & 276 & 1527 & 1424 & 0.850 & 0.150 & 1842 & 0 & 1.000 & 0.150 & 1842 \\
\hline 7 & 66.1 & 7 & 6 & 75.25 & 288 & 1527 & 1424 & 0.887 & 0.113 & 2545 & 0 & 1.000 & 0.113 & 2545 \\
\hline 7 & 66.1 & 8 & 1 & 75.75 & 286 & 1522 & 1476 & 0.868 & 0.132 & 2169 & 0 & 1.000 & 0.132 & 2169 \\
\hline 7 & 66.1 & 8 & 2 & 78.25 & 308 & 1522 & 1476 & 0.859 & 0.141 & 2195 & 0.001 & 0.999 & 0.140 & 2197 \\
\hline 7 & 66.1 & 8 & 3 & 79.75 & 309 & 1522 & 1476 & 0.874 & 0.126 & 2461 & 0 & 1.000 & 0.126 & 2461 \\
\hline 7 & 66.1 & 8 & 6 & 84.25 & 298 & 1522 & 1476 & 0.865 & 0.135 & 2211 & 0 & 1.000 & 0.135 & 2211 \\
\hline
\end{tabular}

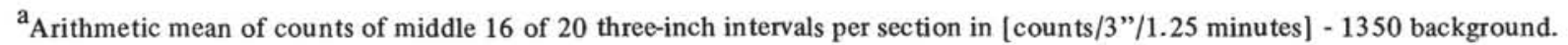




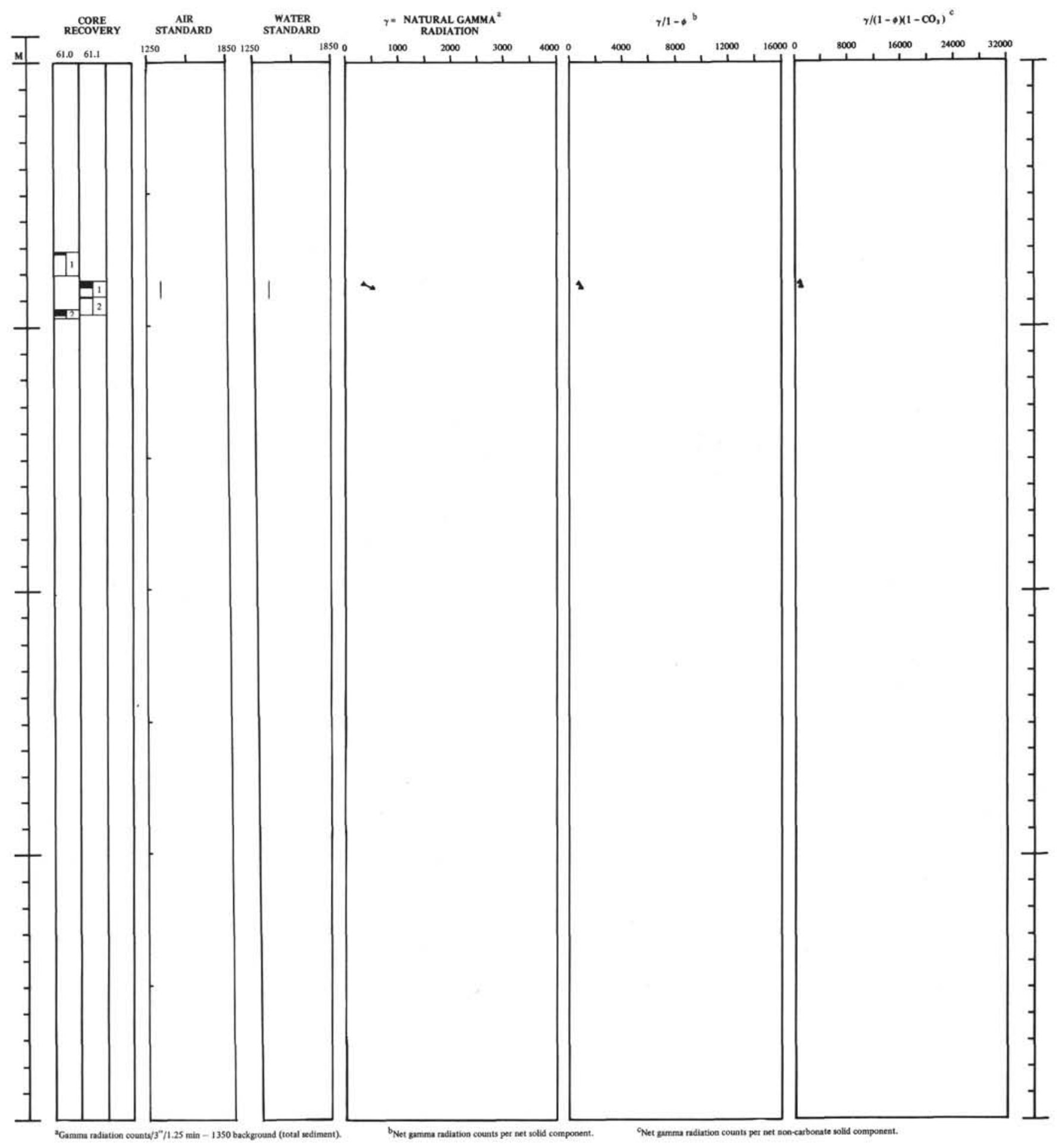

gore 1. Natural gamma radiation, Site 61.

1059 


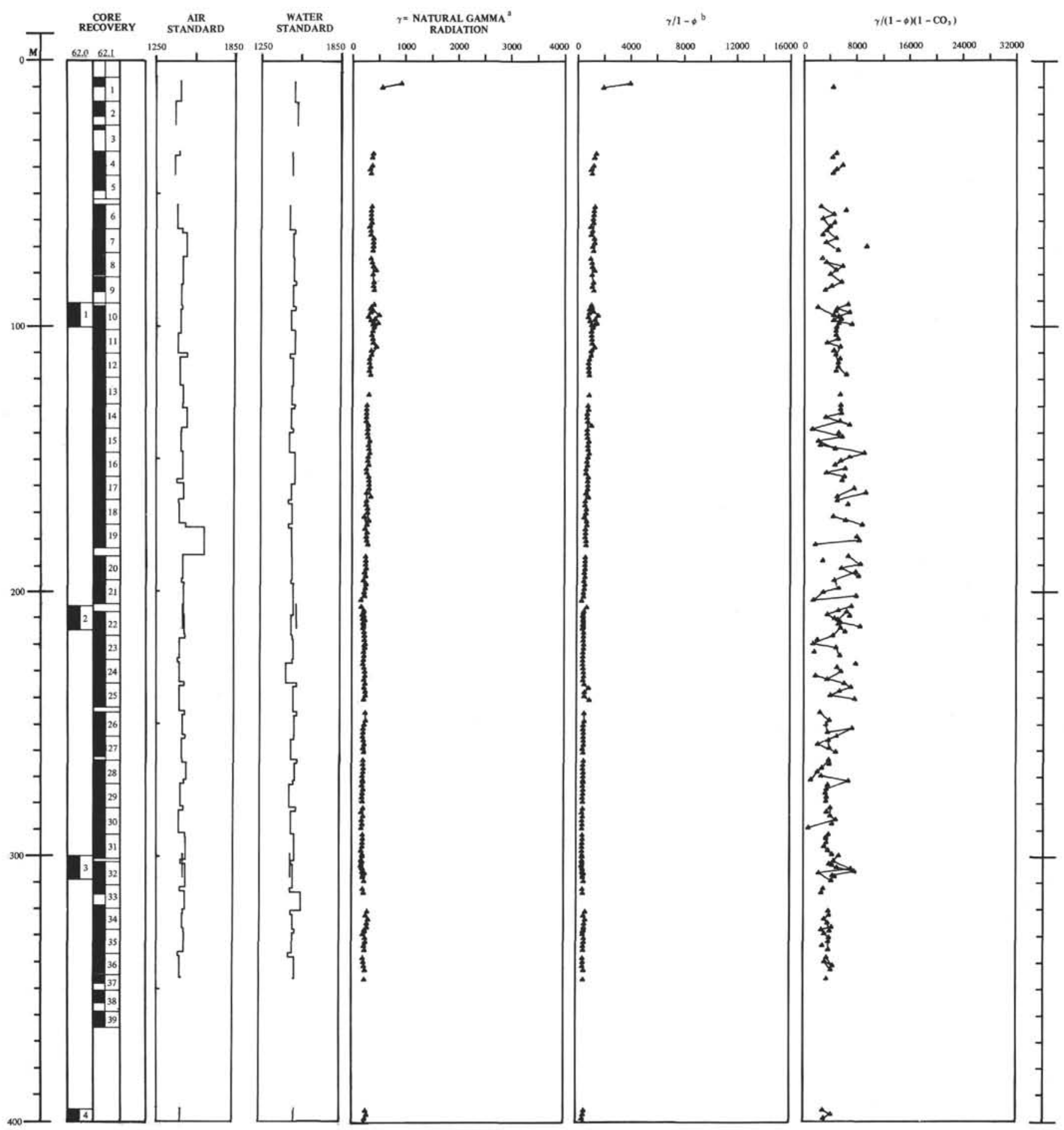

Figure 2. Natural gamma radiation, Site 62. 


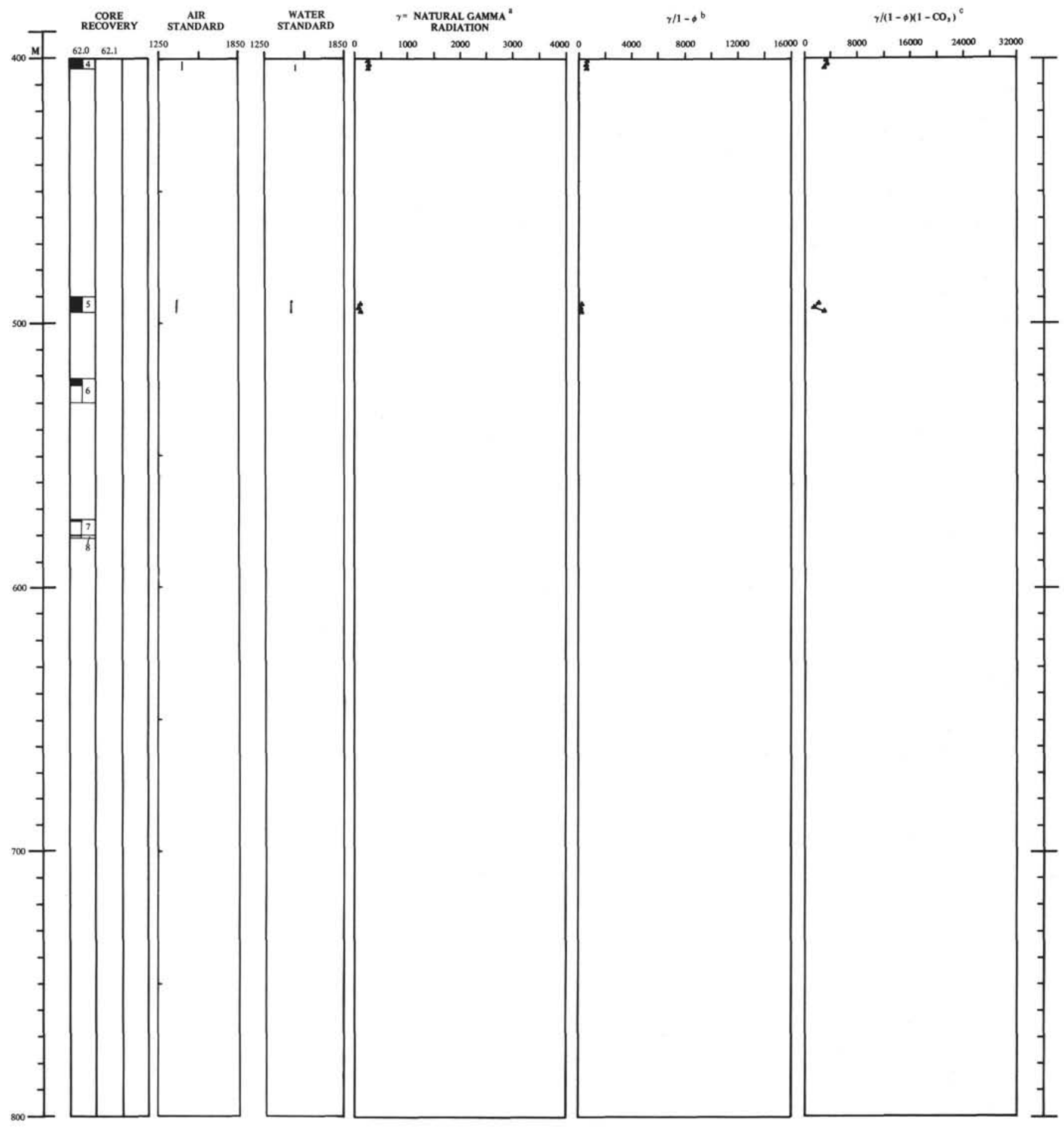

Figure 2. Continued.

1061 


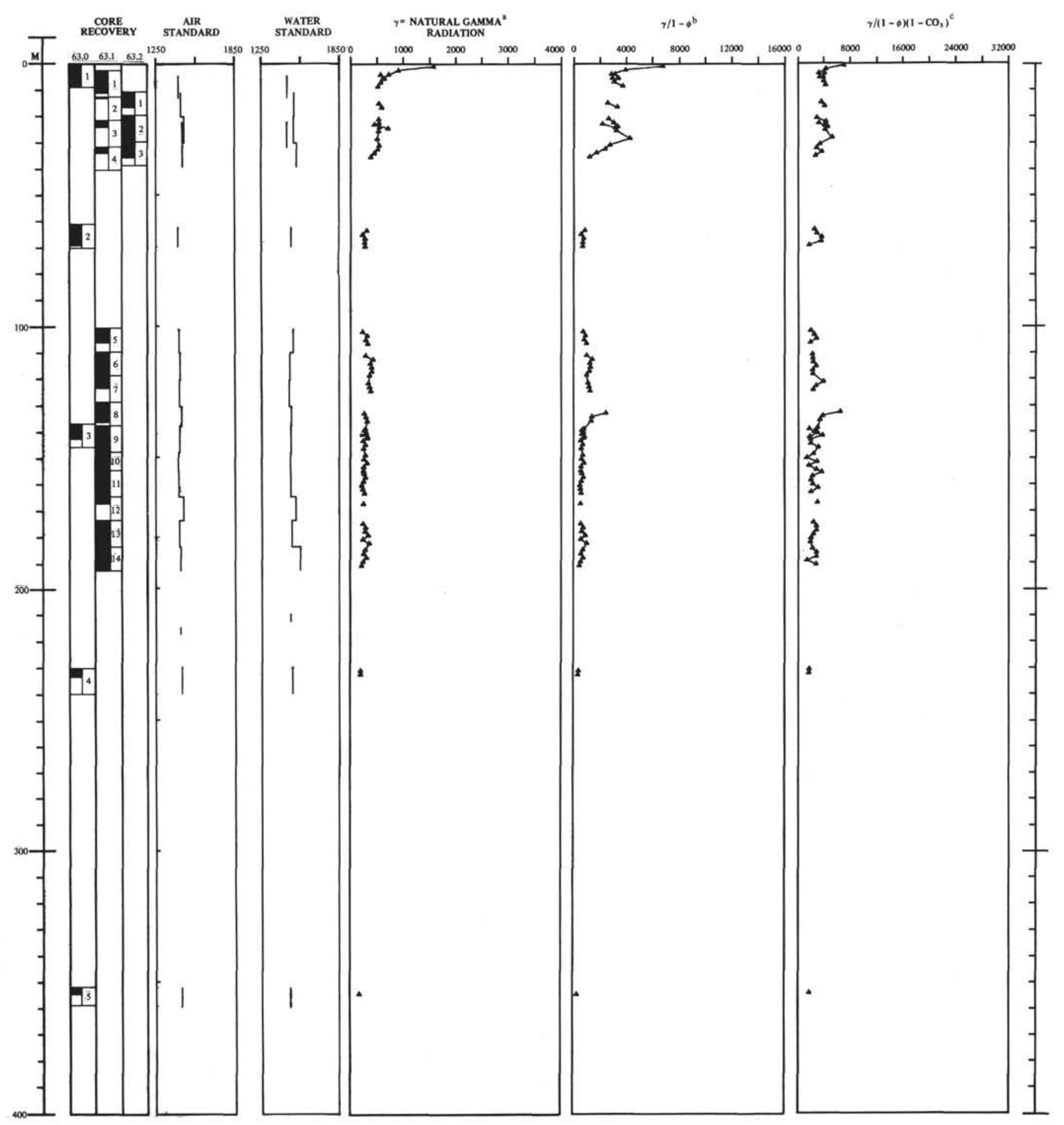

Figure 3. Natural gamma radiation, Site 63. 


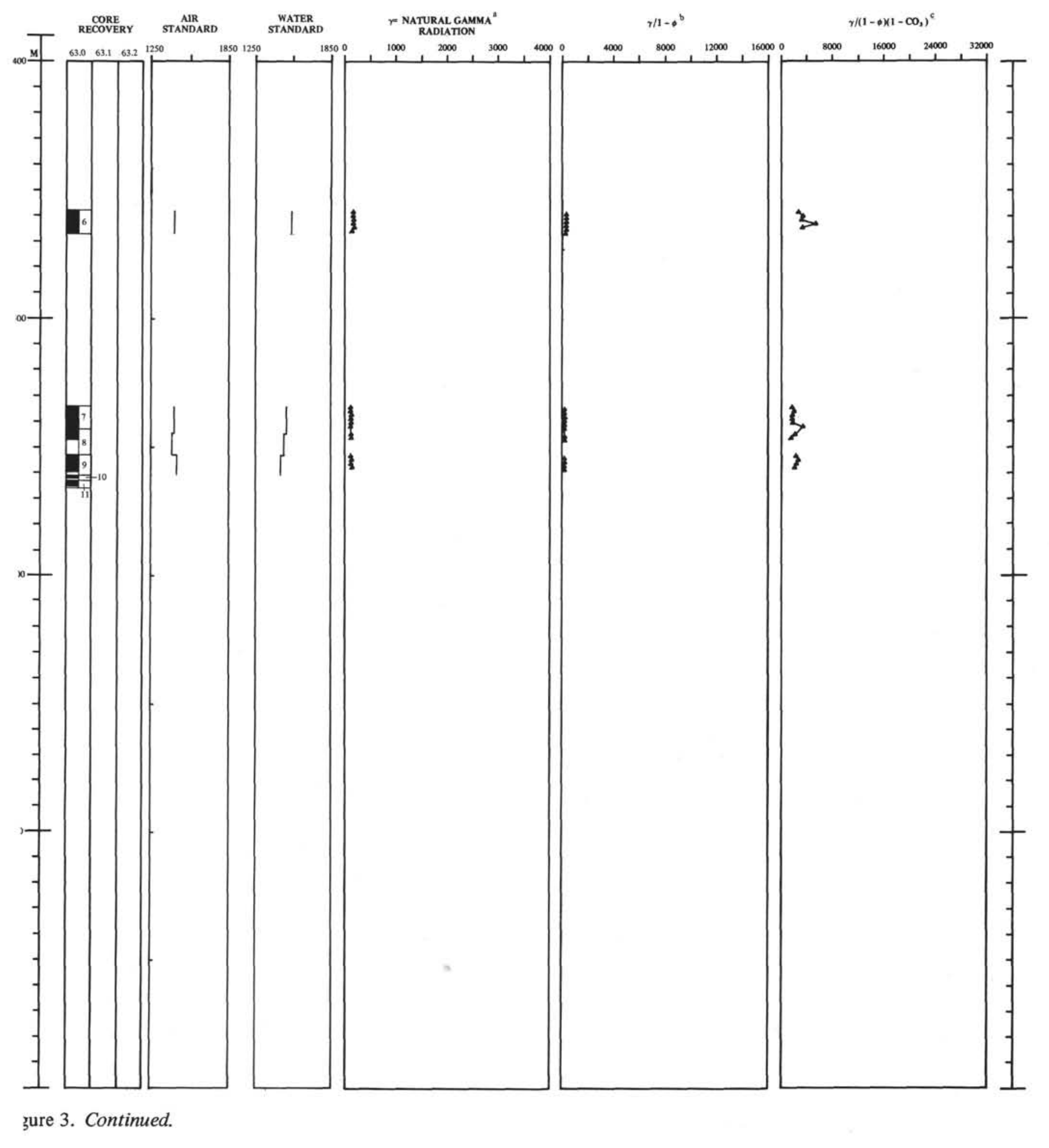

1063 


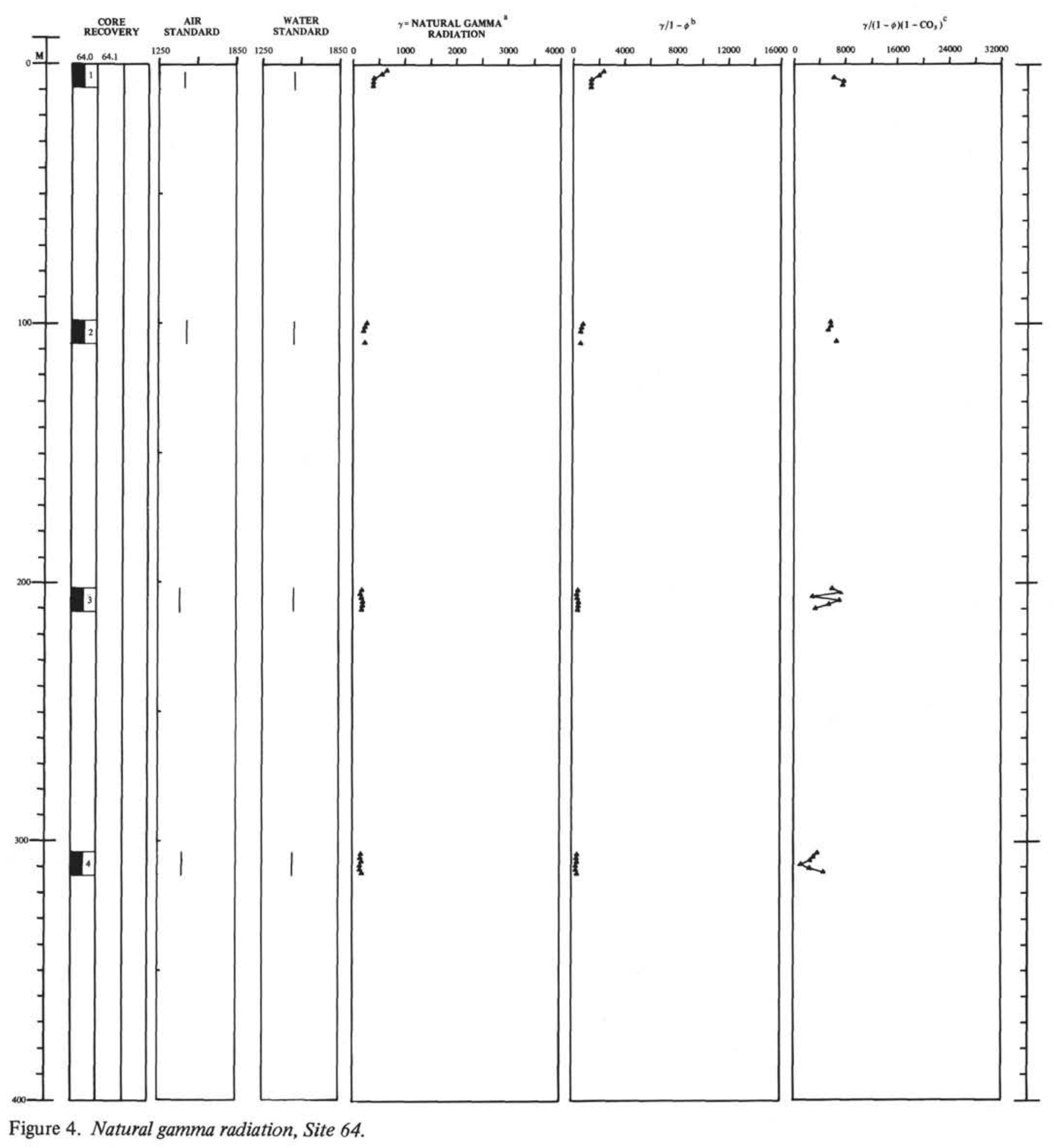

1064 


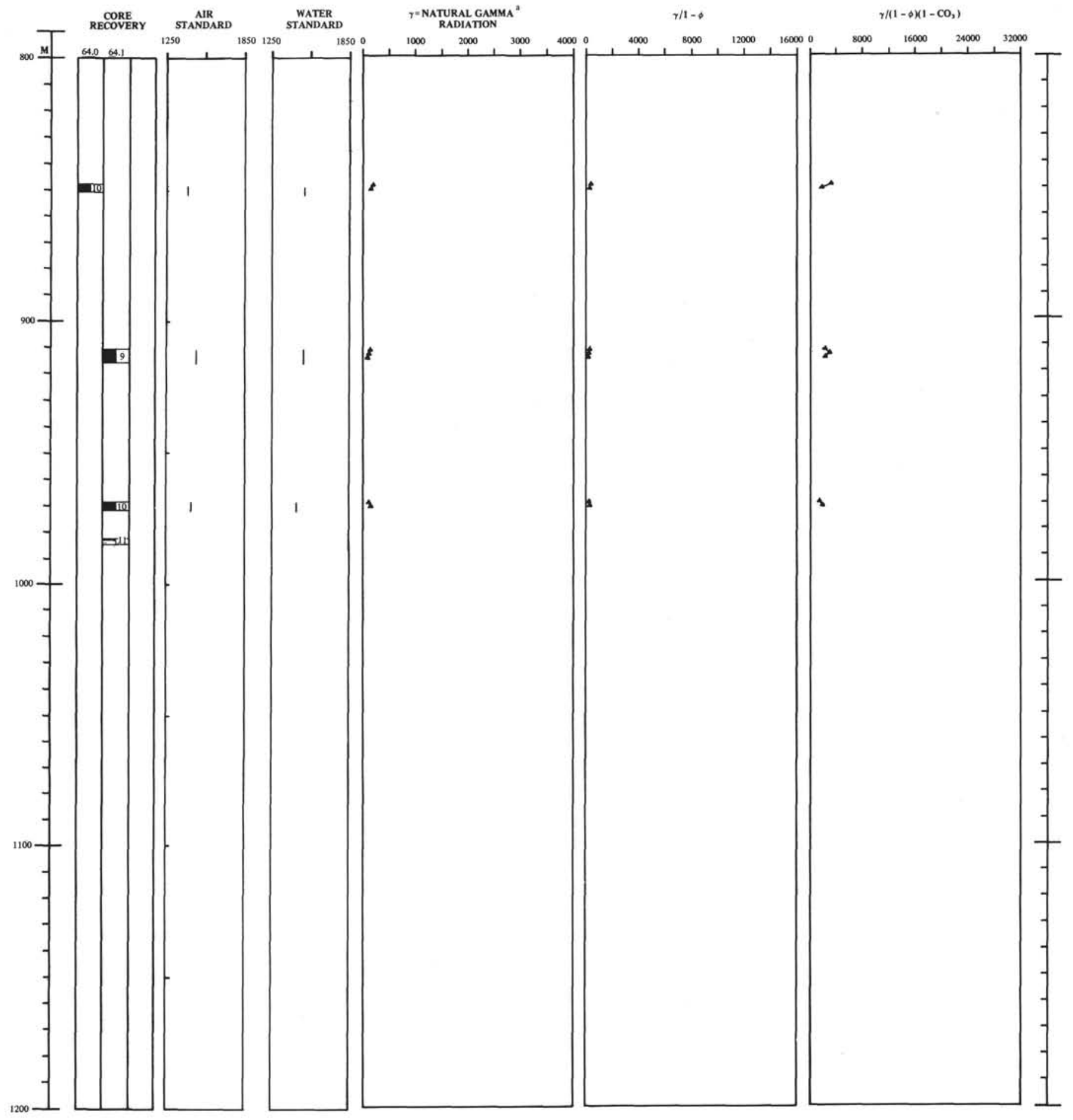

Figure 4. Continued.

1066 


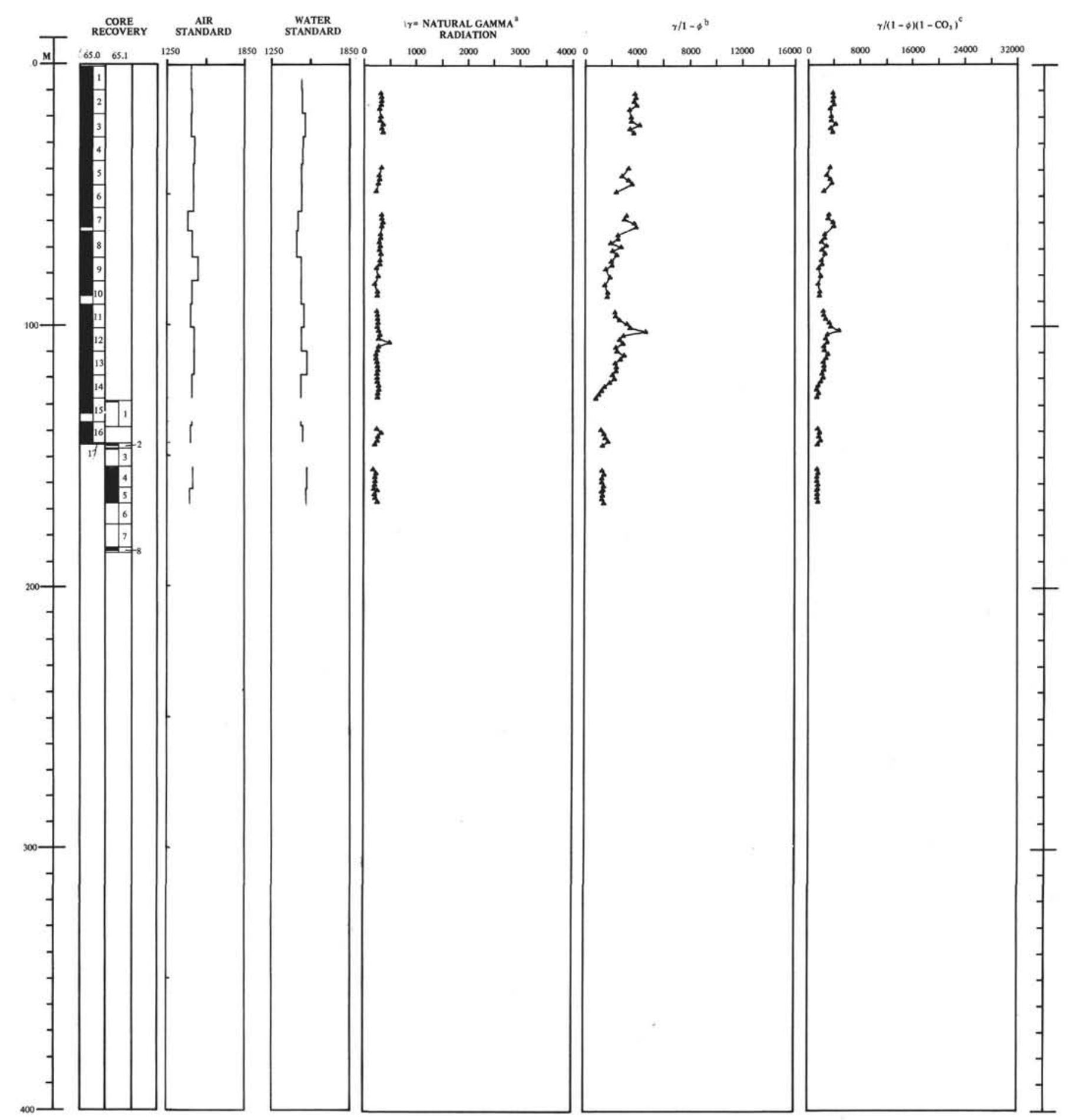

Figure 5. Natural gamma radiation, Site 65. 


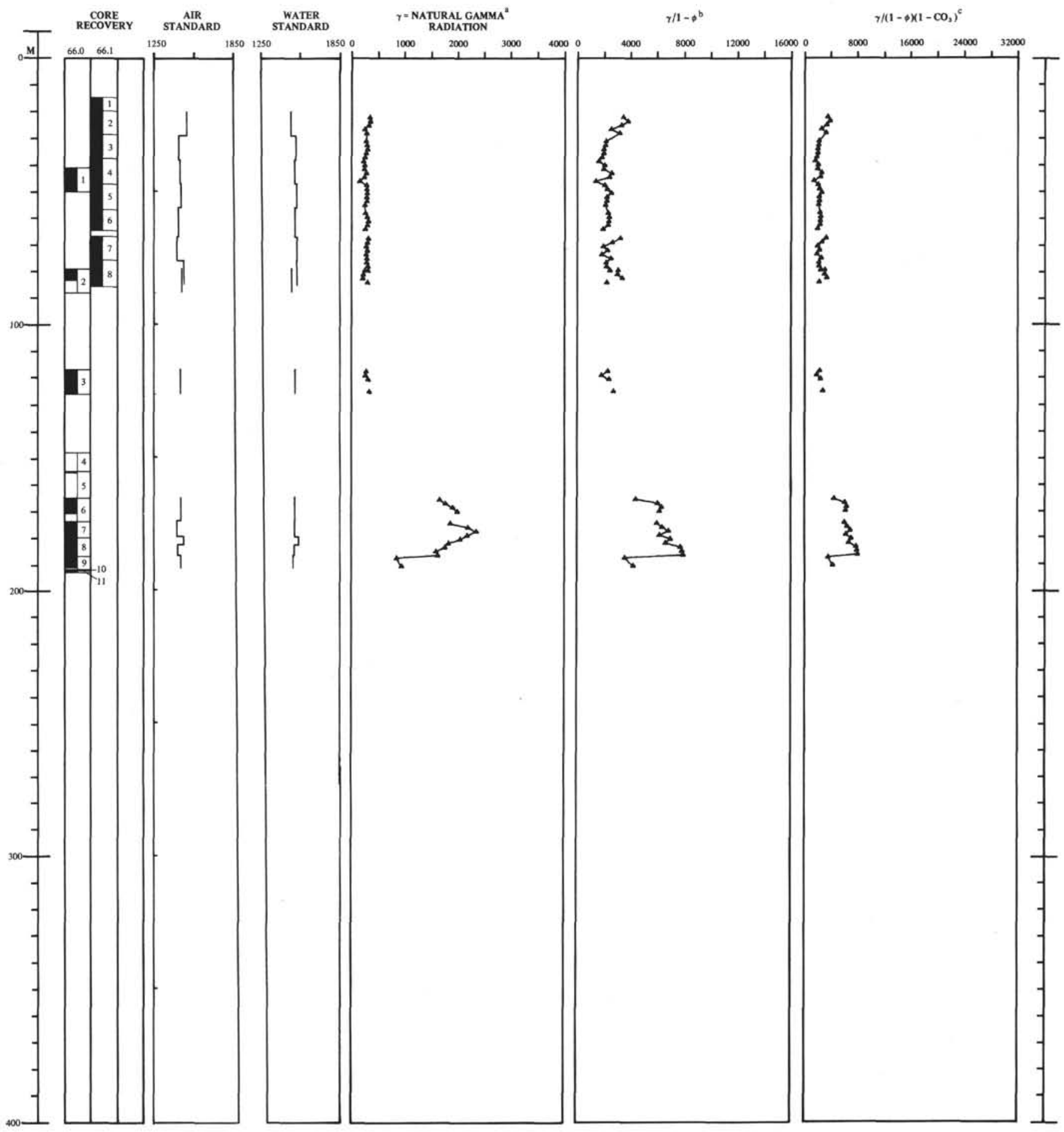

Figure 6. Natural gamma radiation, Site 66.

1068 
correlated with natural gamma radiation measurement on cores, it was found that:

$$
\gamma_{\mathrm{C}}=1350+40.80 \gamma_{\mathrm{L}}
$$

where $\gamma_{C}$ is in counts $/ 3 \times 0.4$-inch core $/ 1.25$ minutes, and $\gamma_{\mathrm{L}}$ is in API units, At Site 29, the mean air control values ranged from 1365 to 1582 counts, a range similar to that obtained on Leg 7. The difference between the derived background value of 1350 from Site 29 data and the air control values may represent the shielding effect of the core material itself.

The uncertain reliability of the controls run in connection with gamma radiation measurements made aboard the D /V Glomar Challenger poses a problem in the selection of a background count by which raw measurements should be reduced. That the system is counting energy from sources other than from the sediment cores is clear. That the cores themselves provide some shielding against extraneous radiation in the laboratory seems likely. Attenuation of only seven per cent of the gamma rays providing the air background count of 1450 would result in the background count of 1350 derived from Leg 4 data. Because variations in the controls appear not to be reflected in the measurements on the cores themselves, we have elected to subtract a constant from the raw data, rather than either of the controls measured between cores. Further, because of the possible shielding effect of the cored materials, we have elected to subtract the minimum value of 1350 counts/1.25 minutes rather than the median values of either the water or air controls.

Thus, all raw gamma-ray data collected on Leg 7 in total counts have been corrected by subtracting a value of 1350 counts, and these corrected values are designated net counts. Net gamma ray counts are shown as a function of depth on the Core description Charts and the Site Summaries in Chapters 3 through 9. Values of net gamma counts as a function of depth are also shown on Figures 1 through 6 of this chapter.

The average of the net gamma-ray counts of the middle 16 to 20 increments in each section scanned were averaged. These averages are plotted on Figures 1 through 6 and are listed in Table 1. Values of average net gamma radiation are repeated in the tables of physical and chemical properties at the end of Chapters 3 through 9 .

Like other down-hole gamma ray tools in current use, the gamma ray tool used on Leg 4 was calibrated in test pits maintained by the American Petroleum Institute (A.P.I., 1959; W. B. Belknap et al., 1966). The tools are calibrated in API units wherein the tool response is set to zero in a nonradioactive pit, and set to respond about 11.7 API units for each microgram of radium equivalent per metric ton.

If $\quad \gamma_{\mathrm{R}}=\begin{aligned} & \text { natural gamma ray radiation of } 1 \text { micro- } \\ & \text { gram radium equivalent per ton, }\end{aligned}$

and $\gamma_{\mathrm{R}}=11.7 \gamma_{\mathrm{L}}$

and $\gamma_{C}=1350+40.8 \gamma_{L}$,

then $\gamma_{R}=.2878 \gamma_{C}-387$.

The following concentrations of radioactive materials give about the same response as one microgram of radium per ton: $2.8 \mathrm{ppm}$ uranium, $3.5 \mathrm{ppm}$ thorium, and $2000 \mathrm{ppm}$ potassium.

The volume of the sediment mass to which the natural gamma scanner is responding is uncertain. If it responds to the entire volume and only to the volume represented ny the 2.5 -inch diameter core, 3 inches in length, 322 cubic centimeters are involved. Not considering attenuation by core materials, a net count of 650 would require a concentration of 2.65 per cent potassium, which is not unreasonable for some marine clays.

The amount of attenuation of the natural gamma radiation provided by the core material itself cannot be known in the absence of information about the energy spectra. However, if the gamma radiation is from the decay of $\mathrm{K}^{40}$, at least 70 to 80 per cent of quanta with the original energy should be recorded by the crystals (Figures 11 and 12). This estimate is based on the following assumptions:

1) the photon energy of the radiation is $1.4 \mathrm{Mev}$;

2) the source of the radiation is a line at the axis of the cylindrical core;

3) the radius of the core is 3.125 centimeters;

4) the centimeters of material necessary to reduce the gamma rays by a factor of two is (after L. Slack, and K. Way, 1959):

$$
\begin{aligned}
& \text { water }\left(\rho=1.00 \mathrm{gm} / \mathrm{cm}^{3}\right): \mathrm{T}_{1 / 2}=11.7 \text { centimeters; } \\
& \text { concrete }\left(\rho=2.35 \mathrm{gm} / \mathrm{cm}^{3}\right): \mathrm{T}_{1 / 2}=5.5 \text { centimeters; } \\
& \text { lead }\left(\rho=11.29 \mathrm{gm} / \mathrm{cm}^{3}\right): \mathrm{T}_{1 / 2}=1.15 \text { centimeters. }
\end{aligned}
$$

In actual fact, many of the attenuated gamma rays will reach the crystals and be counted, and the fractions shown on Figures 11 and 12 should be considered 
minimum values only. Half thicknesses in grams per square centimeters can be derived from the relationship:

$$
\mathrm{T}_{1 / 2}\left(\mathrm{gm} / \mathrm{cm}^{2}\right)=\mathrm{T}_{1 / 2}(\mathrm{~cm}) \rho\left(\mathrm{gm} / \mathrm{cm}^{3}\right) .
$$

\section{RESULTS}

\section{Relationship between Natural Gamma Radiation and Solid Fraction}

It can be observed (Site Summaries, Chapters 3 through 9) that over short stratigraphic intervals the natural gamma radiation tends to decrease with an increase in porosity. In order to test the hypothesis that gamma ray producing elements may be confined largely to the solid phase of these marine sediments, the average net gamma count per section was plotted against the proportion of the total sediment occupied by solid constituents (1-0). The relationships for all sections at each site are shown on Figure 7; the relationships for all sections at all sites is shown on Figure 8.

A least squares regression of the data yields an equation of the form:

$$
\begin{aligned}
\mathrm{Y} & =\mathrm{A} \times+\mathrm{B} \\
\text { where } \quad \mathrm{X} & =(1-\phi), \text { or the net solid component } \\
\text { and } \mathrm{Y} \times 10^{3} & =\text { net gamma counts } / 1.25 \text { minutes }
\end{aligned}
$$

which was calculated for all pairs of samples at Sites $62,63,64,65$ and 66 and for pairs at all of these sites. The standard deviation of $\mathrm{A}$ and $\mathrm{B}$ and the standard error of estimate for $\mathrm{X}$ and $\mathrm{Y}$ were determined, (Table 2). Lines determined by least squares regression are shown on Figures 7 and 8. Statistical techniques are after Dixon and Massey (1957).

Of the comparisons of arrays, those from Sites 62, 63 and 64-where lithologies are similar and the range of net solid components relatively wide-are most credible. The least squares regressions of the other arrays are of questionable value: Data from Site 66 are from both the radiolarian ooze and the pelagic clay; similarly, the composite least squares regression includes data from several different lithologies. Because of the very narrow range of net solid fraction of the radiolarian ooze from Sites 65 and 66 , the least squares regression of these data is questionable. Values of net solid fraction are from about 0.10 to 0.20 and the standard error of estimate for (1-0) in the radiolarian ooze at Site 65 is 0.05 .

Data from Sites 62, 63 and 64 show a decrease in gamma radiation with increase in fraction of solid components, which is in conflict with the observation over short intervals mentioned above. This decrease probably does not indicate a direct relationship, but may result from the fact that an overall decrease in natural gamma radiation with age is noted in rocks of similar type cored on this leg, and the fact that there is a general decrease in porosity with depth.

The net gamma count of the solid fraction of the sediments is listed for each section in Table 1, and is shown plotted as a function of depth in Figures 1 through 6 .

Gamma radiation of the net solid component displayed as a function of depth (Figures 1 through 6) shows a similar pattern at Sites 62,63 and 64 . The radiolarian ooze penetrated at Site 65 and Site 66 shows a much lower gamma radiation to solid fraction ratio than calcareous oozes of similar porosity. However, the gamma radiation of the pelagic clay penetrated at Site 66 is markedly higher $(\times 7)$ than that of either the radiolarian oozes or the calcareous oozes of similar porosity. According to Dreever (Chapter 17, this volume) the clay consists of about 40 per cent palygorskite, 30 per cent mica, 20 per cent montimorillonite, 5 per cent kaolinite and chlorite, and 3 per cent quartz.

\section{Relationship between Natural Gamma Radiation and Noncarbonate Solid Component}

Arrhenius and Goldberg (1955) note that the beta activity of a pelagic carbonate sediment based on its calcium-carbonate free weight is similar to that of nearby carbonate free pelagic clays. To test whether the radionuclides in these marine sediments might be confined largely to the noncarbonate solid component, the average net gamma count per section was plotted against the total net noncarbonate solid fraction of the section $\left[\gamma\right.$ net/ $\left.(1-0)\left(1-\mathrm{CaCO}_{3}\right)\right]$, and the results for sediments at Sites 62,63 and 64 are shown on Figure 8.

Sites 62, 63 and 64 were selected because most of the biogenous component in the cores consists of calcium carbonate.

A least squares regression of the data yields an equation of the form:

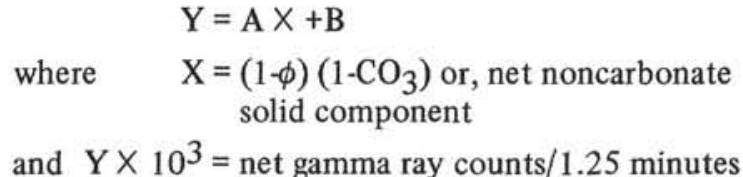

which was calculated for all pairs of samples at Sites 62,63 and 64 and for all pairs at all of these sites. The standard deviation of $\mathrm{A}$ and $\mathrm{B}$ and the standard error of estimate for $\mathrm{X}$ and $\mathrm{Y}$ were determined, (Table $3)$. Lines determined by least squares regression are shown on Figure 9. 
TABLE 2

Comparison of Arrays of Values of Net Solid Component and Net Gamma Count

\begin{tabular}{|c|c|c|c|c|c|c|c|c|}
\hline \multirow[b]{3}{*}{ Site } & \multirow[b]{3}{*}{$\begin{array}{l}\text { Dominant } \\
\text { Lithology }\end{array}$} & \multirow[b]{3}{*}{$\begin{array}{l}\text { No. } \\
\text { Pts. }\end{array}$} & \multicolumn{4}{|c|}{$\begin{array}{l}\text { Values of A and B Calculated by Least } \\
\text { Squares Regression for } \mathrm{Y}=\mathrm{A} X+\mathrm{B}\end{array}$} & \multicolumn{2}{|c|}{$\begin{array}{l}\text { Standard Error } \\
\text { of Estimate }\end{array}$} \\
\hline & & & \multicolumn{2}{|r|}{ A } & \multicolumn{2}{|r|}{ B } & $\mathrm{X}$ & $\mathrm{Y}$ \\
\hline & & & Value & Standard Deviation & Value & Standard Deviation & & \\
\hline 62 & Chalk ooze & 194 & -0.8586 & 0.0146 & +0.6181 & 0.0363 & 0.0584 & 0.0681 \\
\hline 63 & Chalk ooze & 94 & -1.0455 & 0.0201 & +0.7248 & 0.0503 & 0.0755 & 0.0722 \\
\hline 64 & Chalk ooze & 76 & -0.7331 & 0.0170 & +0.4814 & 0.0384 & 0.0326 & 0.0445 \\
\hline 65 & Radiolarian ooze & 70 & -0.2245 & 0.0085 & +0.3176 & 0.0605 & 0.0499 & 0.2220 \\
\hline 66 & $\begin{array}{l}\text { Radiolarian ooze } \\
\text { and pelagic clay }\end{array}$ & 59 & +8.0682 & 0.0322 & -0.6419 & 0.1789 & 0.2319 & 0.0287 \\
\hline $\begin{array}{l}\text { Com! } \\
63,6\end{array}$ & $\begin{array}{l}\text { osite, Sites } 62 \text {, } \\
4,65 \text { and } 66\end{array}$ & 493 & -0.4365 & 0.0153 & +0.4624 & 0.0422 & 0.2853 & 0.6536 \\
\hline
\end{tabular}

TABLE 3

Comparison of Arrays of Values of Net Noncarbonate Solid Component and Average Net Gamma Count

\begin{tabular}{|c|c|c|c|c|c|c|c|c|}
\hline \multirow[b]{3}{*}{ Site } & \multirow[b]{3}{*}{$\begin{array}{l}\text { Dominant } \\
\text { Lithology }\end{array}$} & \multirow[b]{3}{*}{$\begin{array}{l}\text { No. } \\
\text { Pts. }\end{array}$} & \multicolumn{4}{|c|}{$\begin{array}{l}\text { Values of } A \text { and } B \text { Calculated by Least } \\
\text { Squares Regression for } Y=A X+B\end{array}$} & \multicolumn{2}{|c|}{$\begin{array}{l}\text { Standard Error } \\
\text { of Estimate }\end{array}$} \\
\hline & & & \multicolumn{2}{|r|}{$\mathrm{A}$} & \multicolumn{2}{|r|}{$\mathrm{B}$} & $\mathrm{X}$ & $\mathrm{Y}$ \\
\hline & & & Value & Standard Deviation & Value & Standard Deviation & \multirow[b]{2}{*}{0.0704} & \multirow[b]{2}{*}{0.1042} \\
\hline 62 & Chalk-ooze & 194 & +0.6760 & 0.0052 & +0.2289 & 0.0675 & & \\
\hline 63 & Chalk-ooze & 94 & +3.0712 & 0.0193 & -0.0269 & 0.1545 & 0.1075 & 0.0350 \\
\hline 64 & Chalk-ooze & 76 & -0.1876 & 0.0063 & +0.1707 & 0.0972 & 0.0383 & 0.2042 \\
\hline \multicolumn{2}{|c|}{$62,63,64$ (Composite) } & 384 & +1.6453 & 0.0056 & +0.1358 & 0.0622 & 0.1115 & 0.0678 \\
\hline
\end{tabular}

Scatter in these data are high, which is not surprising considering the laboratory difficulties in measuring carbonate (see chapter on carbonate content, this volume) and the generally low level of gamma radiation of these cores. Furthermore, the overall decrease in net gamma radiation with depth undoubtedly obscures any relationship between net gamma count and net noncarbonate solid fraction. Nevertheless, it does appear that there is a correlation: the net gamma radiation increases in general with increased net noncarbonate solid fraction of the sediments.

The net gamma count of the noncarbonate solid fraction of the sediments $\left[\gamma\right.$ net/ $\left.(1-0)\left(1 \mathrm{CO}_{3}\right)\right]$ is listed for each section from Sites 62,63 and 64 in Table 1, and is shown plotted as function of depth for these sites in Figures 1 through 6 . The net gamma count of the noncarbonate solid fraction for Sites 62, 63 and 64 is somewhat lower but of the same order of magnitude as that of the net solid fraction of the pelagic clay penetrated at Site 66 . Values from Site 62 range from 1370 counts near the surface to 2000 counts at about 500 meters; values from Site 63 range from 4190 counts near the surface of 2400 counts at 560 meters; and values from Site 64 range from 12,500 counts near the surface to 1790 counts at 970 meters. Values of net gamma radiation of the net solid fraction of the pelagic clay at Site 66 range from 6000 to 8000 counts. 


\section{RESULTS}

\section{Natural Gamma Radiation of Cores from Site 61}

At Site 61 only 7 meters of core were recovered, and only Sections 1 and 2 of Core 61.1-1 were undisturbed enough to justify measuring natural gamma radiation. The cores were of an Upper Cretaceous stiff clay containing fragments of consolidated porcelanite. The mean net gamma count of Section 61.1-1-1 was 344 , and that of Section 61.1-1-2 was 519 (Figure 1).

\section{Natural Gamma Radiation from Site 62}

Holes at Site 62 penetrated 581 meters of very pure nannofossil ooze-chalk with a sugary dolomite at the base. Sediments ranged in age from Quaternary to Late Oligocene. Almost all sections retrieved from both Holes 62.0 and 62.1 were scanned and a composite from the two sites yields a good profile (Figure 2 ) from a depth of 5 meters to the total depth of 581 meters.

Three zones are detectable on the profile of total net gamma radiation: The first is a Late Quaternary zone of higher radioactivity (as high as an average of 918 counts in Section 62.1-1-2). The top of the second zone is between 10 and 35 meters below the sea floor (early Quaternary) and extends to 110 meters (Early Pliocene), where radioactivity is moderate (about 375 counts). In the third zone the radioactivity decreases steadily from about 330 at a depth of 120 meters (Early Pliocene) to about 105 counts at 490 meters (Early Miocene). The higher counts in the two upper zones may be related in part to lower carbonate content: The carbonate content averages about 60 per cent in the upper zone, about 75 per cent in the middle zone, and about 85 per cent in the lower zone.

Net gamma radiation exceeds 650 net counts at the following horizons: $62.1-1-2$, entire section $>650$ net counts; $62.1-1-3,23-30$ centimeters; $62.1-1-3,84-91$ centimeters; $62.1-1-3,99-106$ centimeters; $62.1-10-3$, 38-61 centimeters; and, $62.1-10-5,137-150$ centimeters.

These layers are probably more radioactive because they contain more volcanogenic material. Volcanic glass was noted (rare or common) in smear slides from Hole 62.0 (Cores 1, 2, 3, 4, 7, 8, 9, 10,11 and 15) and from Hole 62.1 (Cores 2, 3 and 5) but no systematic investigation was made to determine the correlation between natural gamma radiation and volcanogenic content.

The net gamma radiation to net solid fraction also shows high radioactivity in the near surface Quaternary. It decreases steadily from 1370 counts at 35 meters beneath the sea floor to 200 counts at 500 meters, a decrease of 60 counts/M.Y. Fifty per cent of the net gamma radiation (on a net solid basis) is lost after about 11 million years.

The net gamma radiation to net noncarbonate solid fraction increases slightly from about 5000 counts at 35 meters beneath the seafloor to 5600 counts at 130 meters. Late Miocene sediments show both a generally higher gamma count (the average net counts of several sections exceeds 8000 counts) and there is more scatter in these data than in older and younger sediments. Below this interval, the net noncarbonate solid component shows a more or less steady decrease in gamma radiation from 3500 counts at 250 meters to 2000 counts at 500 meters.

\section{Natural Gamma Radiation of Cores from Site 63}

Three holes at Site 63 penetrated 566 meters of sediment consisting of about 30 meters of Quaternary Pliocene pelagic clay and marl overlying a very pure nannofossil ooze chalk ranging in age from Late Miocene to Early Oligocene. The upper thirty meters and the interval between 100 and 190 meters were cored continuously, but the rest of the section was cored only about once each 100 meters.

Despite the incomplete coverage, several zones of radioactivity can be noted. Cores from the PlioceneQuaternary marl-clay sequence are highly radioactive. The uppermost section just below the sea floor shows an average of 1580 net counts; the remainder of the section shows about 500 net counts. Cores from the underlying Miocene nannofossil chalk ooze show a relatively constant gamma radiation of about 250 net counts throughout most of the section. The interval between 100 and 125 meters is slightly more radioactive (as high as 428 ) and the cores at 230 meters show a net gamma radiation of only 193 counts. Below this the net gamma radiation decreases steadily from 193 counts at 230 meters to about 130 counts at 555 meters.

The net gamma radiation to net solid fraction is also high in cores from the Pliocene-Quaternary marl-clay sequence. The uppermost section shows an average of 6775 net counts, and cores from the remainder of the sequence about 3000 counts. Below this interval, the net solid fraction shows a steady decrease from about 670 counts at 65 meters to 215 counts at 555 meters, except for one Middle Miocene interval between 110 and 135 meters which shows a net count of about 1280 .

Except for a layer at the surface and at 135 meterswhich show higher values, the net gamma radiation to net noncarbonate solid fraction shows a steady decrease in net gamma count from 4190 counts at about 10 meters to 1770 counts at 355 meters, in the Upper 
Oligocene. Average net gamma counts of the net noncarbonate solid fraction of cores from the interval below 355 meters are generally higher: about 3450 counts at 460 meters, and about 2400 counts in the interval between 535 to 560 meters.

\section{Natural Gamma Radiation of Cores from Site 64}

Holes drilled at Site 64 penetrated 985 meters of very pure nannofossil chalk ooze ranging in age from Quaternary to Middle Eocene.

As at Sites 62 and 63 , the near surface sediments are more radioactive. Measurements on cores indicate that radioactivity of the ooze decreases steadily from an average of 230 net counts at 100 meters beneath the sea floor (Lower Pliocene) to 122 net counts at 500 meters (Lower Miocene). Below this the net gamma count is nearly constant to the total depth.

The net gamma radiation to solid fraction is also high for cores taken in the first 7 meters. Below this interval, the net gamma counts to solid fraction decrease steadily from 660 net counts at 100 meters to 270 counts at 500 meters. Below this, the net gamma counts to solid fraction remains relatively constant to the total depth.

The net gamma radiation to net noncarbonate solid fraction is also high above 7 meters $(12,500$ counts). Below this it decreases irregularly with depth from 7500 at 10 meters, to 5500 at 100 meters, to 5400 at at 200 meters, to 3000 at 300 meters. Below 300 meters the ratio remains about the same to a depth of 750 meters. Below this it decreases steadily to 1790 counts at a depth of 970 meters.

\section{Natural Gamma Radiation of Cores from Site 65}

Holes at Site 65 penetrated 187 meters of radiolarian ooze ranging in age from Quaternary to Middle Eocene.

The average net gamma radiation of cores from the radiolarian ooze decreases steadily from 340 counts at 10 meters to 225 counts at 160 meters. One unusually radioactive section (65.0-12-4) had an average net count of 503. The surface sediments were not cored.

The average net gamma radiation to solid fraction decreases in a more erratic manner. At 10 meters the count is 3800 and it decreases more or less regularly through Pliocene and Upper Miocene sediments to about 3100 in a Middle Miocene ooze at 57 meters. Below this, the count decreases abruptly to 1600 at 85 meters. Near the Lower Miocene-Upper Oligocene boundary, the counts increase to an average of about 3000 counts, and it is this interval that contains the radioactive layer mentioned above. Below this boundary, the average net gamma counts decrease to about 1400 and are near this value in the underlying Eocene sediments.

Except for a few layers of nannofossil-radiolarian ooze and impure silicified limestone between 125 to 140 meters, sediments penetrated at Site 65 are almost devoid to carbonate. Thus, the values of average net gamma radiation to net noncarbonate solid fraction are similar to the values of net radiation to solid fraction.

\section{Natural Gamma Radiation of Cores from Site $\mathbf{6 6}$}

Holes drilled at Site 66 penetrated 165 meters of radiolarian ooze ranging in age from Quaternary to Early Miocene, overlying about 25 meters of stiff fine-grained pelagic clay, Cretaceous in age. The natural gamma radiation of the radiolarian ooze is uniformly low, about 300 average net counts throughout. The pelagic clay is highly radioactive, average net counts range from 1630 to 2350 , except in the lowest part. The base of the clay is rich in ferromanganese oxides and volcanoclastic sands, and is less radioactive than the overlying clay, averaging 900 counts.

In the radiolarian ooze, the natural gamma radiation to net solid fraction decreases from about 3500 average net counts at 25 meters to 2000 average net counts at 35 meters, and then increases slightly to 2000 counts at 120 meters. Except for the uppermost section, and intervals rich in ferromanganese oxides at the base, the Cretaceous pelagic clay has a high net gamma count to net solid fraction and it increases from about 4500 counts at 16.5 meters. Several sections in the interval from 70 to 85 meters show over 3000 net counts/net solid fraction.

The sediments penetrated at Site 66 are almost devoid of carbonate, and therefore the values of average net gamma radiation to net noncarbonate solid fraction are almost identical to the values of net gamma radiation to net solid fraction.

\section{DISCUSSION}

One of the several dozen radioactive isotopes occurring in nature, those of the uranium, thorium and protactinium series and potassium-40 produce almost all of the natural gamma radiation of marine sediments (Frielander, et al.). All of the daughter isotopes of these decay series have half lives less than one million years except those of:

$$
\begin{array}{ll}
\mathrm{U}^{238}: & \text { half life }=4.51 \times 10^{9} \text { years; } \\
\mathrm{U}^{235}: & \text { half life }=7.13 \times 10^{8} \text { years; } \\
\mathrm{Th}^{232}: & \text { half life }=1.41 \times 10^{10} \text { years; } \\
\mathrm{K}^{40}: & \text { half life }=1.25 \times 10^{9} \text { years. }
\end{array}
$$


Studies of cores collected on Leg 7 show that where data exist, near surface sediments all have high natural gamma radiation. Values decrease rapidly to a depth equivalent to about 1 million year, where there is a break in the gradient of net gamma count displayed as a function of depth.

Cores of sediments recovered below this surface layer show a consistent and substantial reduction in natural gamma radiation with depth. For example, at Site 62 (Table 4) there is a loss of 69 per cent of the natural gamma radiation between 115 meters and 490 meters. On a total saturated sediment basis, 69 per cent of the natural gamma radiation is lost in 15 million years; on a dry basis, 77.5 per cent is lost.

Until the specific radioactive isotopes producing the gamma radiation and their ratios are determined, speculations concerning the observed decrease in gamma radiation are tenuous.

Some, but not all, of the reduction with depth in gamma radiation counted can be attributed to the increased attenuation of the gamma rays by the increase in density with depth (Figure 12). If the dominant radionuclide is $\mathrm{K}^{40}$, an increase in density from 1.6 to 2.2 would result in a reduction in gamma rays counted of less than 10 per cent.

If the radioactive nuclides were confined to the interstitial water, the reduction in interstitial water content with depth would account for the decrease in natural gamma radiation with depth. However, at Site 62 a reduction in natural gamma radiation of 69 per cent from 115 meters to 490 meters parallels a reduction in porosity of only 22.6 per cent, which is insufficient to explain all of the reduction in radioactivity. Further, over short intervals, the natural gamma radiation appears to increase with a decrease in porosity.

The reduction in natural gamma radiation with depth cannot be due to the decay of radionuclides in the sediments. At Site 62 , on a total saturated sediment basis, 50 per cent of the gamma radiation is lost after 10.8 million years; on a dry basis, 50 per cent is lost after 9.7 million years. The loss in radiation at Sites 63 and 64 also indicate a rate of loss of about 50 per cent per 10 million years. No radioactive nuclides known have a half life which approximates 10 million years.

\section{Changes in Initial Radionuclide Content}

The decrease in gamma radiation with age may be due, at least in part, to a gradual increase with time in the initial radioactivity of the sediments at accumulation. Both the amount of radionuclides delivered to the ocean water mass by subaerial erosion, and the conditions favorable to their incorporation into sediments could vary with time.

The first possibility is supported by a study of sediments at the Guadalupe Site. Chow, Tatsumoto and Patterson (1962) show that the composition of authigenic lead and biogenic lead in sediments from this site changes with time. They state that the older lead, which may have been derived primarily from predominantly basalt-surface drainage during the Middle Miocene, is less radioactive than the average lead in the oceans and that post Miocene lead (derived primarily from drainage from crystalline plutons or their sedimentary equivalents) is more radioactive.

With regard to the second possiblity, there is evidence that changes in bottom circulation may result in changes in radionuclide content of the bottom sediments. Arrhenius (1963) notes that phosphates accumulating under reducing conditions are high in uranium, whereas those accumulating under oxidizing conditions have a low uranium content.

\section{Loss of Radionuclides by Leaching and Advection}

The decrease in radioactivity with depth may reflect gradual leaching and removal of radionuclides by advection of interstitial fluids as the sediments compact. Some earlier studies tend to support this hypothesis.

Arrhenius and Goldberg (1955) analyzed a highly radioactive pelagic clay near the sediment surface, and found that the fine fraction, consisting largely of clay and hydroxide minerals, was as radioactive as a pure phillipsite fraction, in contrast to deeper pelagic clay samples, where the radioactivity was produced mostly by the coarser phillipsite-rich fractions. They conclude that a reduction in beta activity in the fine fraction with age resulted from leaching of unsupported ionium with time.

A study of recent and ancient carbonate sands by Haglund, Freedman and Miller (1969) shows that a consistent decrease in uranium content with consolidation occurs as aragonite and magnesium-rich calcite are removed.

Koczy (1954) found an excess of radium over ionium in seawater and attributed this excess to the addition of uranium to the ocean water mass by diffusion from near surface sediments. Work by Bernat and Goldberg (1969) shows that there are movements of $\mathrm{Ra}^{228}$ within the sedimentary column. 
TABLE 4

Reduction of Natural Gamma Radiation with Depth at Site 62

\begin{tabular}{lccccccc}
\hline \multicolumn{1}{c}{ Core } & $\begin{array}{c}\text { Depth } \\
\text { (meters) }\end{array}$ & Age & $\gamma_{\mathrm{C}}$ & $\rho_{\mathrm{B}}$ & $\phi$ & $1-\phi$ & $\frac{\gamma}{(1-\phi)}$ \\
\hline $62.1-12$ & 115 & 4.8 m.y. & 335 & 1.67 & 0.62 & 0.38 & 880 \\
$62.0-5$ & 490 & 19.5 m.y. & 104 & 1.90 & 0.48 & 0.52 & 200 \\
\cline { 2 - 7 } & 375 & 15 m.y. \pm & $231=69 \%$ & $14 \%$ or $22.6 \%$ & 680 or $77.5 \%$ \\
\hline
\end{tabular}

\section{FUTURE WORK}

Two modifications in the natural gamma radiation program of the Deep Sea Drilling. Project in particular would enhance the usefulness of the data collected considerably:

First, the scanning system should be calibrated with nonradioactive blanks having a range of densities approximating those of cores processed.

Second, some means for determining the energy spectra of the gamma radiation of the cores should be incorporated with the system.

Also, because of the disturbing effect of drilling and coring on recovered materials, an in-hole gamma ray logging program is strongly recommended.

\section{ACKNOWLEDGMENTS}

The author is grateful to Rudolph H. Beiri, Minoru Koide and Sachio Yamamoto who critically read the manuscript and offered a number of suggestions.

\section{REFERENCES}

American Petroleum Institute, 1959. Recommended Practice for Standard Calibration and Form for Nuclear Logs. New York (American Petroleum Institute).

Arrhenius, G. and Goldberg, E. O., 1955. Distribution of radioactivity in pelagic clays. Tellus. 7, 226.

Arrhenius, Gustaf, 1963. Pelagic sediments. In The Sea. New York (John Wiley and Sons).

Arrhenius, G. et al., 1957. Localization of radioactive and stable heavy nuclides in ocean sediments. Nature. $\mathbf{1 8 0}, 85$.

Belknap, W. B., Dewan, J. J., Kirkpatrick, C. V., Motts, W. E., Pearson, A. J., and Rabson, W. R., 1966. A.P.I. calibration facility for nuclear logs. A.P.I. Res. Paper. 33 (Revised).
Bernat, Michel and Goldberg, E. D., 1969. Thorium isotopes in the marine environment. Earth and Planetary Science Letters 5. Amsterdam (NorthHolland Publishing Co.), 308.

Chenouard, Lydie and Lalou, Claude, 1969. GammaRay spectrometry as a tool for a rapid investigation of detritic cores. J. Sediment Petrol. 39 (4), 1477.

Chow, Tsaihwa, Tatsumoto, M. and Patterson, C. C., 1962. Lead isotopes and uranium contents in experimental Mohole cores (Guadalupe Site). $J$. Sediment. Petrol. 866.

Dixon, W. J. and Massey, Jr., F. G., 1957. Introduction to Statistical Analysis. New York (McGraw-Hill Book Co., Inc.).

Evans, H. B. and Lucia, J. A., 1970. Natural gamma radiation sources. In MNA Peterson et al., Initial Reports of the Deep Sea Drilling Project, Volume IL. Washington (U. S. Government Printing Office).

Frielander, Gerbardt, Kennedy, Joseph and Miller, Julian, Nuclear and Radio Chemistry. New York (John Wiley and Sons).

Gealy, E. L. and Gerard, R. D., 1970. In-situ petrophysical measurements in the Caribbean. In Bader, R. G. Initial Reports of the Deep Sea Drilling Project, Volume IV. 1970, Washington (U. S. Government Printing Office).

Goldberg, E. D., 1954. Marine geochemistry, 1. chemical scavengers of the sea. J. Geol. 62, 249.

Goldberg, E. D. and Arrhenius, G. O. S., 1958. Chemistry of Pacific pelagic sediments. Geochim. Cosmochim. Acta. 13, 153.

Haglund, D. S., Friedman, G. M. and Miller, D. S., 1969. The effect of fresh water on the redistribution of uranium in carbonate sediments. $J$. Sediment. Petrol. 39 (4), 1283.

Heath, Russel, 1964. Scintillation spectrometry, gamma ray spectrum catalog. At. Energy Commiss., Res. Develop. Rept. \#IDO-16880-1.

Kaufman, A., 1969. The $\mathrm{Th}^{232}$ concentration of surface ocean water. Geochim. Cosmochim. Acta. 33, 717 . 
Koczy, F. F., 1954. Geochemical balance in the hydrosphere. In Nuclear Geology. H. Faul (Ed.) New York (John Wiley and Sons, Inc.), 120.

Koczy, F. F., Picciotto, E., Paul, G. and Wilgain, S., 1957. Mesure des isotopes du thorium dans l'eau de mer. Geochim. Cosmochim. Acta. 11, 103.

Koczy, F. F., 1958. Natural Radium as a tracer in the ocean. Proc. 2nd Intern. Conf. Peaceful Uses At. Energy. 18, 351.

Koczy, F. F., 1966. Age determination in sediments by natural radioactivity. In The Sea. New York (Interscience Publishers), 3.

$\mathrm{Ku}$, Teh-Lung and Broecker, W. S., 1969. Radiochemical studies on manganese nodules of deep-sea origin. Deep-Sea Res. 16, 625.
Maxwell, A. E. et al., 1970. Explanatory Notes. In Initial Reports of the Deep Sea Drilling Project, Volume III. Washington (U. S. Government Printing Office) 11 .

Moore, W. S., 1969. Measurement of $\mathrm{Ra}^{228}$ and $\mathrm{Th}^{228}$ in sea water. J. Geophys. Res. 74 (2).

Scott, M. R., 1968. Thorium and uranium concentrations and isotope ratios in river sediments. Earth and Planetary Science Letters 4. 245.

Slack, T. and Way, K., 1959. Radiations from radioactive atoms in frequent use. U. S. At. Energy Commiss., Washington, D. C.

Turekian, Karl K., 1964. The marine geochemistry of strontium. Geochim. Cosmochim. Acta. 28, 1479. 

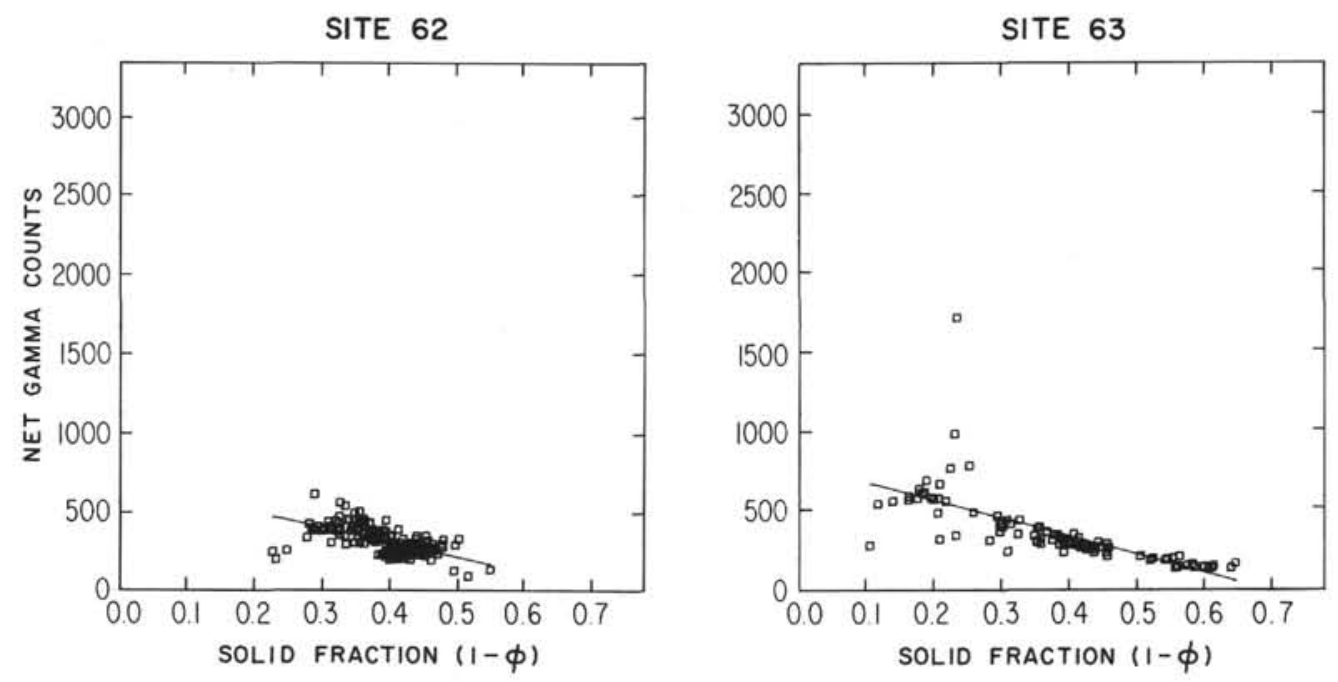

SITE 64

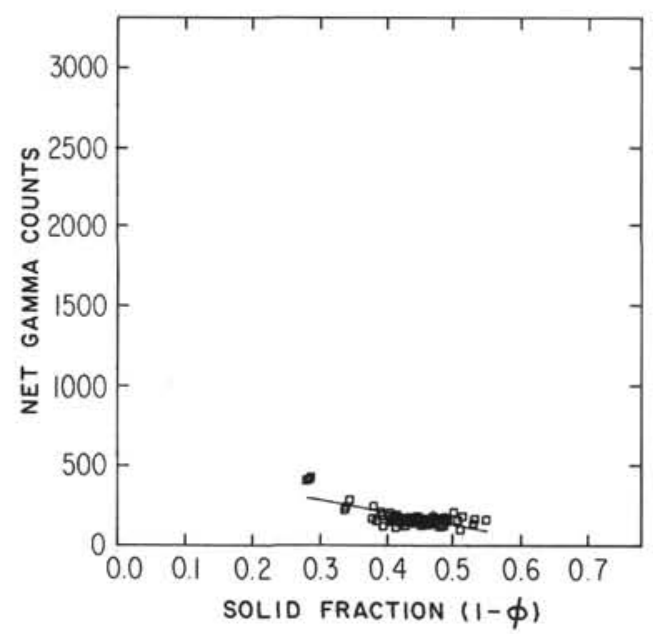

SITE 65
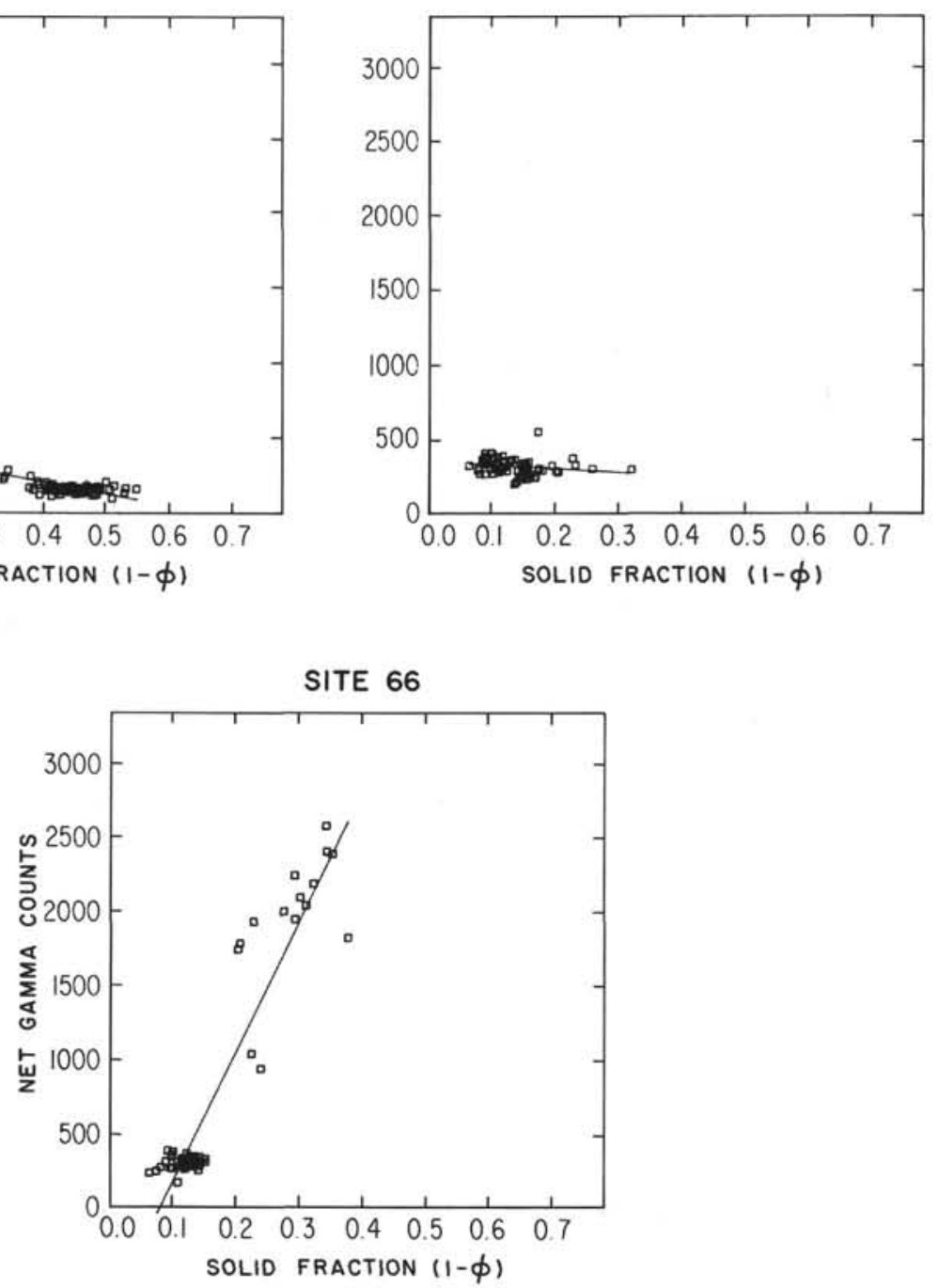

Figure 7. Relationships between natural gamma radiation and net solid fraction for all sections taken at Sites 62, 63, 64, 65 and 66. 


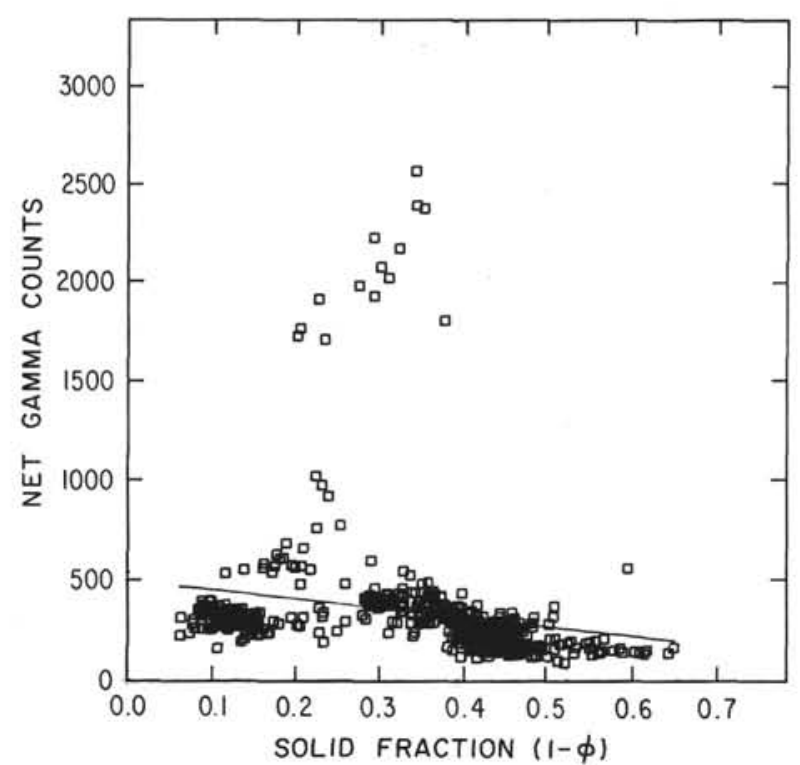

Figure 8. Relationships between natural gamma radiation and net solid fraction for all sections taken at Sites 62 through 66: composite.
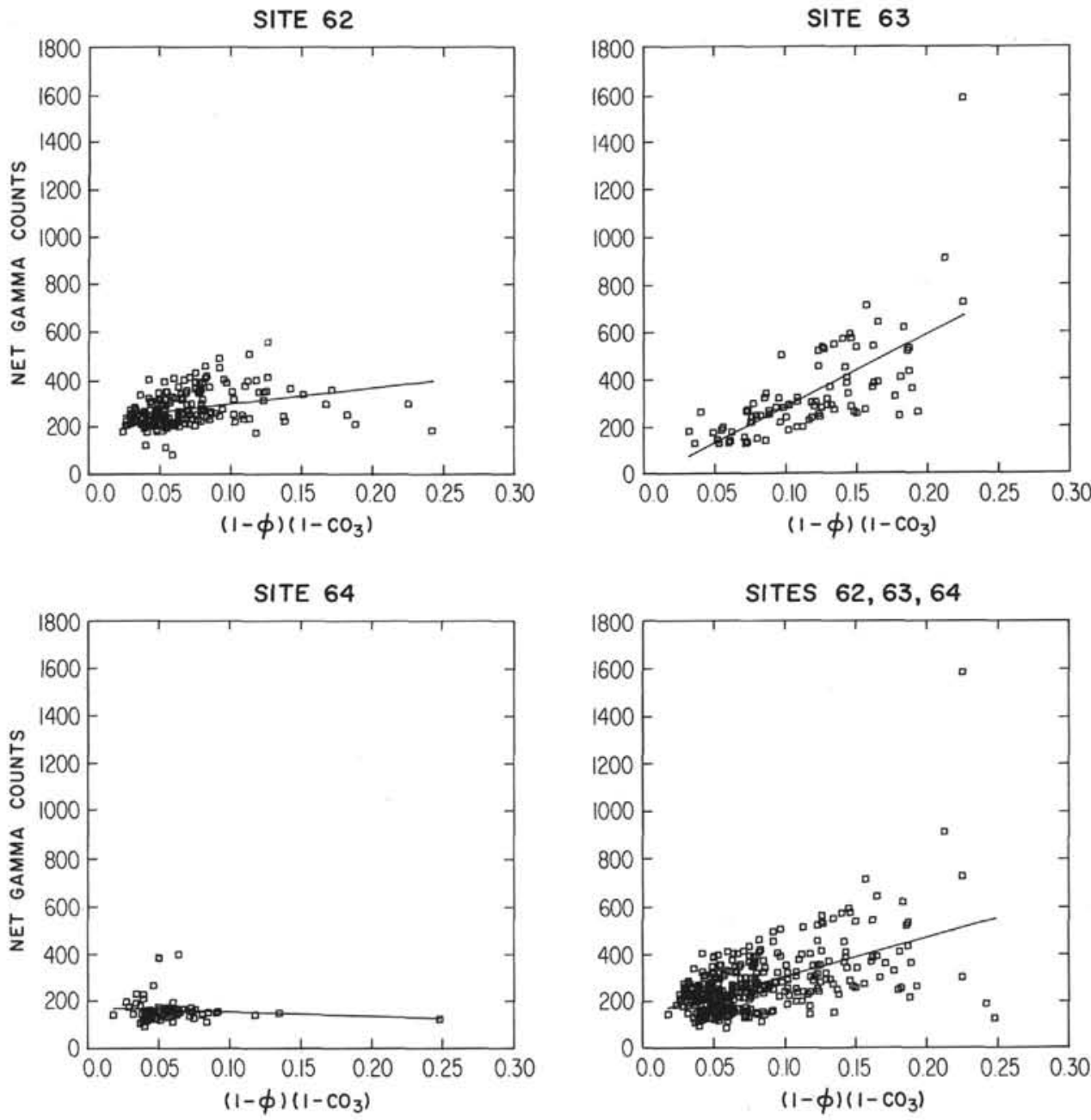

Figure 9. Relationship between natural gamma radiation and net noncarbonate solid fraction for all sections taken at Sites 62,63 and 64 . 


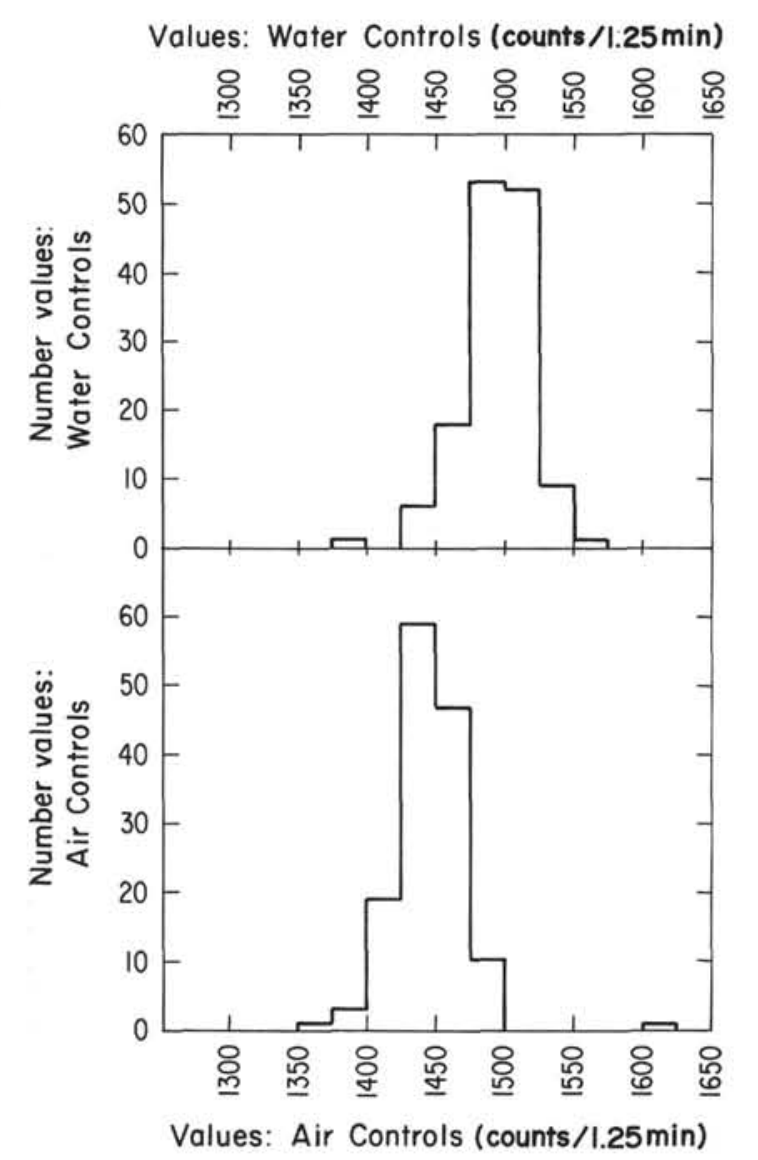

Figure 10. Distribution air and water controls for natural gamma radiation.

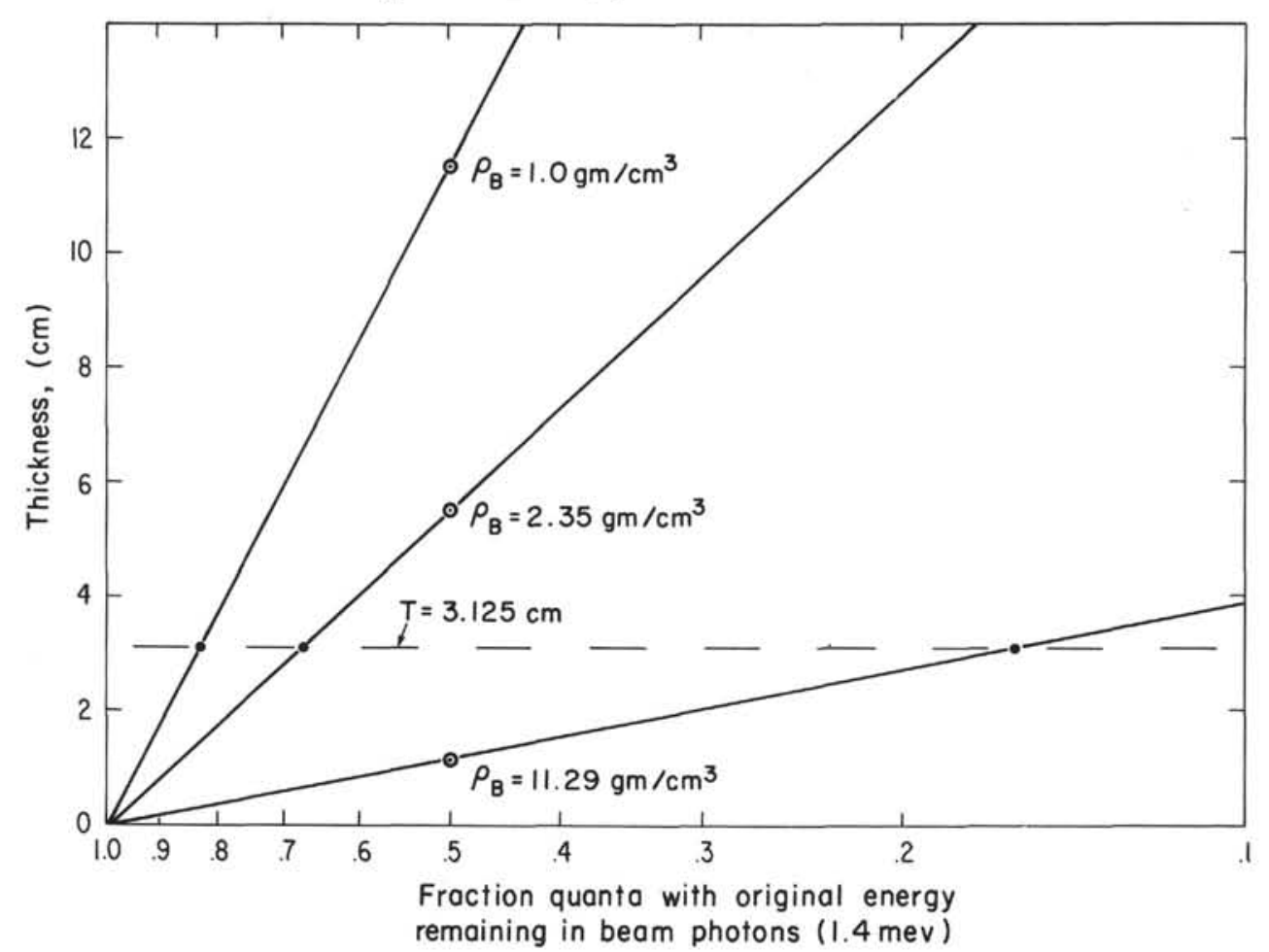

Figure 11. Relationship between percentage original signal at $1.4 \mathrm{Mev}$ remaining and half thickness. 


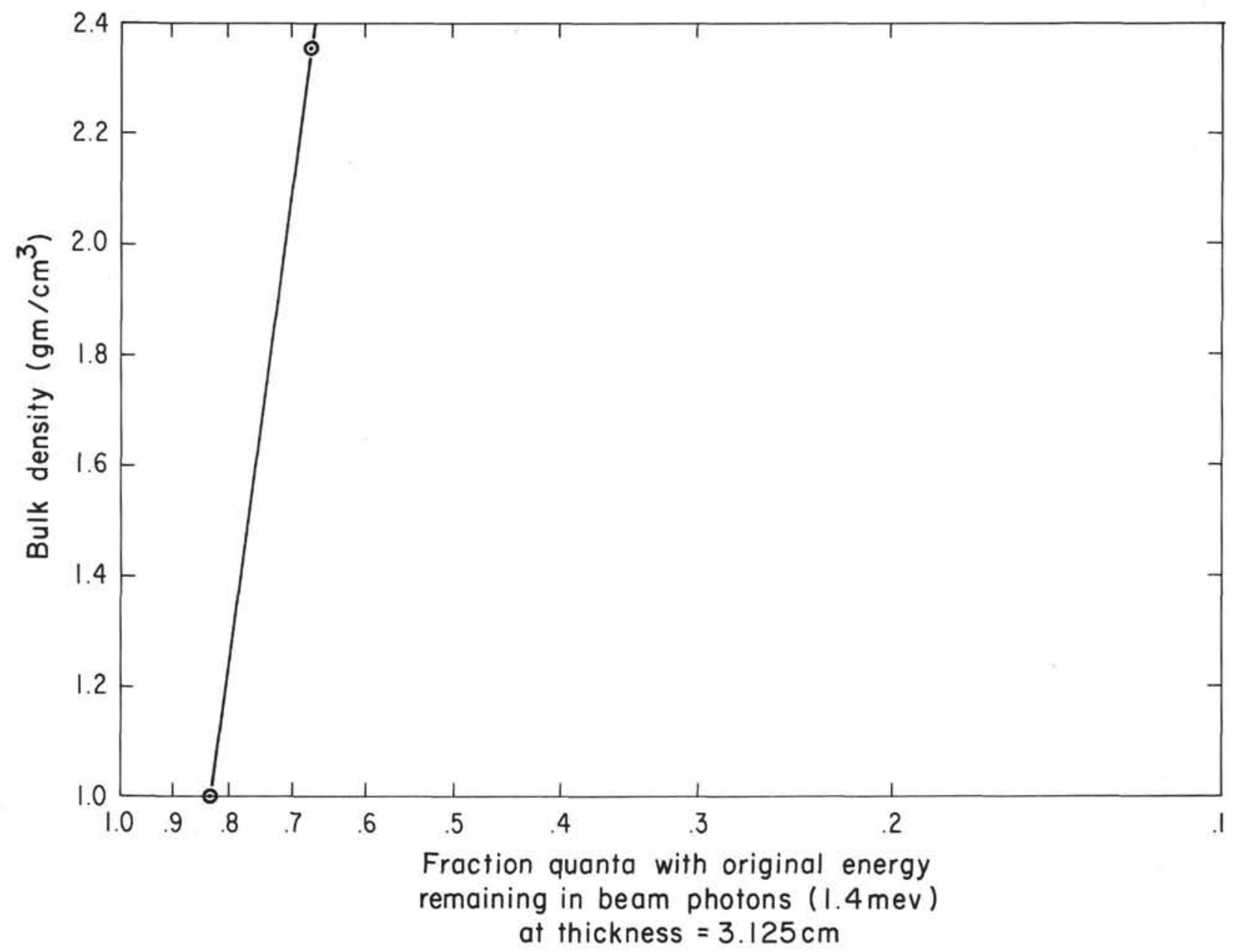

Figure 12. Relationship between bulk density and percentage signal at $1.4 \mathrm{Mev}$ remaining at $T_{1 / 2}=3.125$ centimeters. 